\author{
Universidade de São Paulo \\ Instituto de Física
}

\title{
Incluindo Efeitos da Densidade de Momento Inicial e Tensor de Tensões no Mapeamento da Resposta Hidrodinâmica em Colisões de Íons Pesados
}

\author{
Jefferson Arthur Dias de Sousa
}

Orientador: Prof. Dr. Matthew William Luzum

Dissertação de mestrado apresentada ao Instituto de Física da Universidade de São Paulo, como requisito parcial para a obtenção do título de Mestre em Ciências.

Banca Examinadora:

Prof. Dr. Matthew William Luzum- Orientador (IFUSP)

Prof. Dr. David Dobrigkeit Chinellato (Unicamp)

Prof. Dr. Tiago José Nunes da Silva (UFSC) 

University of São Paulo

Physics Institute

\title{
Including Effects of Initial Momentum Density and Stress Tensor in the Mapping of the Hydrodynamic Response in Heavy Ion Collisions
}

\author{
Jefferson Arthur Dias de Sousa
}

Supervisor: Prof. Dr. Matthew William Luzum

Dissertation submitted to the Physics Institute of the University of São Paulo in partial fulfillment of the requirements for the degree of Master of Science.

Examining Committee:

Prof. Dr. Matthew William Luzum- Supervisor (IFUSP)

Prof. Dr. David Dobrigkeit Chinellato (Unicamp)

Prof. Dr. Tiago José Nunes da Silva (UFSC) 

To Arlete Vilhena

without her, I would not dream to arrive far. . . 



\section{Acknowledgements}

Foremost, I would like to express my big thanks to God, he was extremely generous to me. None of this would have been possible without him.

I would like to express my deepest gratitude to my Supervisor, Prof. Dr. Matthew Luzum. His immerse knowledge, constant support, and expert advise were inspiring motivations throughout my studies. I have been extremely lucky to have a supervisor who cared so much about my work, with his immediate answers to my questions, constructive criticism, and patience. My research and this thesis would not have been possible without him. Thank you very much!

I would like to express my big thanks to my parents João Sousa and Deize Dias for all support given by them, and to family and friends for be here with me all the time.

Special thanks must go to the my research colleagues for all contributions.

I would like to thank the Rice University by provide a guest account for I use the cluster DAVinCI, and to Professor Jorge Noronha Junior and Jean-Yves Ollitrault by contribution in my research.

This study was financed in part by the Coordenação de Aperfeiçoamento de Pessoal de Nível Superior - Brasil (CAPES) - Finance Code 001. 

God does not play dice with the Universe.

Albert Einstein 



\section{Abstract}

The evolution of a heavy-ion collision system deals in many stages, where one of them is known as Quark-Gluon Plasma (QGP) which behaves as a relativistic fluid and therefore all properties of standard hydrodynamic are valid, and consequently the evolution of the system can be entirely determined by the initial conditions. In other words, the anisotropic flow is well understood as a hydrodynamic response to spatial anisotropies in the system density at early time. This response function can be written as a systematic expansion in terms of length scales, such that the known quantity called eccentricity represents the global structure and contains initial features of the system to predict the final observables represented by the harmonic flows. However, the initial conditions for hydrodynamics consist of an energymomentum tensor as well as any conserved current, and components such as momentum density and stress tensor can also contribute. Although they are thought to be less important than energy density, their effects should have increasing importance for smaller collision systems on flow observables. A framework able to include these effects was constructed, along with numerical tests from full hydrodynamic simulations to demonstrate its efficacy.

Keywords: QGP; hydrodynamics; framework; simulations. 



\section{Resumo}

A evolução de um sistema de colisão de íons pesados lida com muitos estágios, onde um deles é conhecido como Plasma de Quarks e Glúons (PQG) o qual se comporta como um fluido relativístico e portanto todas as propriedades da hidrodinâmica padrão são válidas e, consequentemente, a evolução do sistema pode ser inteiramente determinada pelas condições iniciais. Em outras palavras, a anisotropia final de fluxos é bem entendida como uma resposta hidrodinâmica às anisotropias espaciais no sistema de densidade em um tempo inicial. Esta função de resposta pode ser escrita como uma expansão sistemática em termos de escalas de comprimento, tal que a quantidade conhecida como excentricidade representa a estrutura global e contém características iniciais do sistema para prever os observáveis finais representados pelos fluxos harmônicos. Entretanto, as condições iniciais para hidrodinâmica consistem no tensor de energia e momento tal como qualquer corrente conservada, e componentes como a densidade de momento e o tensor de tensões podem contribuir. Embora eles sejam vistos como menos importantes que a densidade de energia, seus efeitos em observáveis finais de fluxo devem ter um aumento de importância para sistemas de colisões menores. Uma estrutura capaz de incluir esses efeitos foi construída, e através de testes numéricos provenientes de simulações hidrodinâmicas completas foram feitos para demonstrar sua eficácia.

Palavras-chave: PQG; hidrodinâmica; estrutura; simulações. 



\section{List of Figures}

1.1 The time evolution of a typical heavy ion collision from MADAI collaboration, Hannah Petersen and Jonah Bernhard. . . . . . . . . . . . . . 2

1.2 A typical heavy ion collision. Figure adapted from [29]. . . . . . . . . . . . 4

1.3 In (left), we have the success of the hydrodynamic modelling of the QGP (the red line crossing the experimental dots). The IP+Glasma is the initial condition model, whilst MUSIC is the hydrodynamic code that models the space-time evolution of the QGP. In the (rigth), we have the experimental coefficients $V_{n}$ are in good agreement with the theoretical model for $\eta / s=$ 0.2 . Figure adapted from $[30] \ldots \ldots$. . . . . . . . . . . .

1.4 Impacter parameter, spectors and participants presented in Ref. [37]. . . . .

1.5 Schematic representation of a nucleus-nucleus collision in the $(z, t)$ plane. The thick lines are the trajectories of the colliding nuclei, which are moving nearly at the velocity of light. The lines of constant $z / t$ are also lines of constant $\eta_{s}$ made at Ref. [39]. . . . . . . . . . . . . . . . . .

3.1 Examples of initial energy density at midrapidity for a $\mathrm{Pb}+\mathrm{Pb}$ collision, where its structure on the (left) has a more granular distribution with local details and on the (right) has a smoother aspect. Figure from [65]. . . . . .

3.2 Profile of initial energy density at midrapidity symmetrically distributed in the transversal plane because $a_{n}=0 \ldots \ldots \ldots \ldots$

3.3 Profile of energy density deformed because $a_{2} \neq 0$ (left) and deformed because $a_{3} \neq 0$ (right). . . . . . . . . . . . . . . .

3.4 Relation between real projections of $V_{2}$ and $\epsilon_{2}$ event-by-event. . . . . . . .

3.5 Relation between real projections of $V_{3}$ and $\epsilon_{3}$ event-by-event. . . . . . . .

4.1 Profile of momentum density symmetrically distributed because $b_{n}$ and $c_{n}$ are zero. . . . . . . . . . . . . . . . . . . . .

4.2 Profile of momentum density asymmetrically distributed because $b_{2} \neq 0$ (left) and because $b_{3} \neq 0$ (right). . . . . . . . . . . . . . .

4.3 Profile of momentum density asymmetrically distributed because $c_{2} \neq 0$ (left) and because $c_{3} \neq 0 \ldots \ldots \ldots \ldots \ldots$

4.4 Plots of $V_{2} \times \epsilon_{2}(\alpha=0)$ (upper left), of $V_{2} \times \epsilon_{2}(\alpha=2.0)$ fm (upper right), of $V_{2} \times \epsilon_{2}(\alpha=5.0) \mathrm{fm}$ (lower left), and of $V_{2} \times \epsilon_{2}(\alpha=9.0) \mathrm{fm}$ (lower right). 33

4.5 Relation between $V_{3}$ and $\epsilon_{3}(\alpha)$ event-by-event. . . . . . . . . . .

4.6 Profile of magnitude of a symmetrically distributed stress tensor because $p_{n}=0 \ldots \ldots \ldots \ldots \ldots \ldots \ldots$ 
4.7 Profile of a asymmetrically distributed stress tensor because $p_{2} \neq 0$ (left)

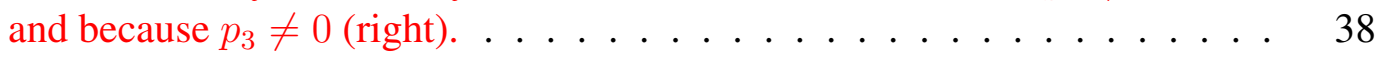

4.8 Plots of $V_{2} \times \epsilon_{2}(\beta=0)$ (upper left), of $V_{2} \times \epsilon_{2}(\beta=2.0) \mathrm{fm}$ (upper right), of $V_{2} \times \epsilon_{2}(\beta=4.0) \mathrm{fm}$ (lower left), and of $V_{2} \times \epsilon_{2}(\beta=6.0) \mathrm{fm}$ (lower right). 39

4.9 Relation between $V_{3}$ and $\epsilon_{3}(\beta) \ldots \ldots \ldots \ldots$. . . . . . . . 40

4.10 Relation between $V_{2}$ and $\epsilon_{2}(\alpha, \beta)$ event-by-event. . . . . . . . . . . . 42

4.11 Relation between $V_{3}$ and $\epsilon_{3}(\alpha, \beta)$ event-by-event. . . . . . . . . . 43

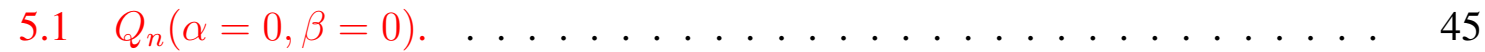

5.2 Response coefficient $\kappa_{n}$ for different centralities. . . . . . . . . . . . . 46

$5.3 Q_{n}(0,0)$, and $Q_{n}\left(\alpha_{\text {best }}, \beta_{\text {best }}\right) . \ldots \ldots \ldots \ldots \ldots \ldots$

$5.4 Q_{n}(0,0), Q_{n}\left(\alpha_{\text {best }}, \beta_{\text {best }}\right)$, and $Q_{n}\left(\alpha=2.0 \mathrm{fm}, \beta=20.0 \mathrm{fm}^{2}\right) \ldots \ldots \ldots$ 


\section{List of Tables}

3.1 Parameters of initial energy density symmetrically distributed. . . . . . . . 24

3.2 Parameters used for $V_{2} \times \epsilon_{2} \ldots \ldots \ldots \ldots \ldots \ldots$

3.3 Parameters used for $V_{3} \times \epsilon_{3} \ldots \ldots \ldots \ldots \ldots \ldots$

4.1 Parameters used for momentum density. . . . . . . . . . . . . . . . . . . . 31

4.2 Parameters used for $V_{2} \times \epsilon_{2}(\alpha) \mathrm{fm} \ldots \ldots \ldots \ldots \ldots \ldots$

4.3 Parameters used for $V_{3} \times \epsilon_{3}(\alpha) \ldots \ldots \ldots \ldots \ldots$

4.4 Parameters used for magnitude of stress tensor. . . . . . . . . . . . . 37

4.5 Parameters for $V_{2} \times \epsilon_{2}(\beta) \ldots \ldots \ldots \ldots \ldots \ldots$

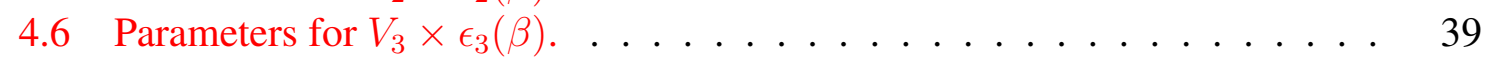

4.7 Parameters used for $V_{2} \times \epsilon_{2}(\alpha, \beta) \ldots \ldots \ldots \ldots \ldots \ldots \ldots \ldots \ldots \ldots \ldots$

4.8 Parameters used for $V_{3} \times \epsilon_{3}(\alpha, \beta) \ldots \ldots \ldots \ldots \ldots$

5.1 Best values of $\alpha$ and $\beta$ for $n=2 \ldots \ldots \ldots \ldots$. . . . . . . . 47

5.2 Best values of $\alpha$ and $\beta$ for $n=3 \ldots \ldots \ldots \ldots$. . . . . . . . 47 



\section{Contents}

1 Introduction 1

1.1 Heavy ion Physics . . . . . . . . . . . . . . . . . . . 1

1.2 The viscosity of the QGP . . . . . . . . . . . . . . . . . 3

1.3 Initial condition to final state mapping . . . . . . . . . . . . . 4

1.4 Participants, spectators, and impact parameter ............... 6

1.5 Centrality ......................... 7

1.6 Rapidity and pseudorapidity . . . . . . . . . . . . 7

2 Model description 9

2.1 Relativistic hydrodynamics . . . . . . . . . . . . . . . . . . 9

2.1 .1 Ideal hydrodynamics . . . . . . . . . . . . . . . . . . . . . 10

2.1.2 Viscous hydrodynamics ........................... 11

2.1.3 Before hydrodynamics . . . . . . . . . . . . . . . . 12

2.1 .4 After hydrodynamics . . . . . . . . . . . . . . . . . . . . 13

2.2 Relativistic kinetic theory of gases . . . . . . . . . . . . . . . . . . . 14

2.3 Final hadron dynamics . . . . . . . . . . . . . . . . 16

3 Mapping the hydrodynamic response 17

3.1 Characterizing the initial stage of a heavy-ion collision . . . . . . . . . 17

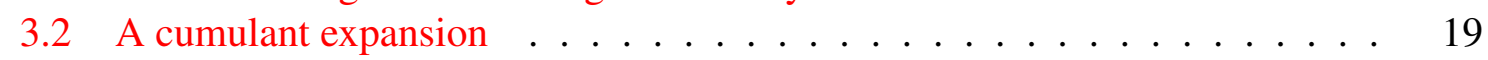

3.3 Energy density as a generating function . . . . . . . . . . 23

4 Including effects from other $T^{\mu \nu}$ components 27

4.1 Physical motivation . . . . . . . . . . . . . . . 27

4.2 Effects from initial momentum density . . . . . . . . . . . 28

4.3 Effects from initial Stress tensor . . . . . . . . . . . . . . . . . . 35

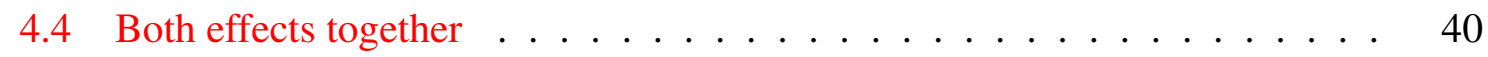

5 Realistic simulations $\quad \mathbf{4 4}$

5.1 Usual estimator . . . . . . . . . . . . . . . . . . 45

5.2 Estimator with new contributions . . . . . . . . . . . 46

6 Conclusions $\quad 49$

A Derivation of cumulants $\quad 5$ 
B Developing the generating function $\mathbf{5 5}$

B.1 Including momentum density . . . . . . . . . . . . . . . 55

B.2 Including stress tensor $\ldots \ldots \ldots \ldots \ldots$ 


\section{Chapter 1}

\section{Introduction}

\subsection{Heavy ion Physics}

The study of ultra-relativistic heavy ion collisions is an interdisciplinary field which involves high-energy physics of elementary particles and nuclear physics [1]. The name "heavy-ion" is used for large atomic nuclei, whereas the term "ultra-relativistic" denotes a regime where the kinetic energy exceeds significantly the rest energy. Here, high-energy particle physics is referred by ultra-relativistic energies and nuclear physics is referred by atomic nuclei that collide.

A nucleus is comprised of protons and neutrons, and protons and neutrons are basically made of quarks that are confined by a strong interaction mediated by gluons that hold the quarks together, and this interaction has been described by Quantum Chromodynamics ${ }^{1}$ (QCD) [2].

Imagine that we have a resistant box that cannot be opened easily, and we are interested to figure out what there are inside it. The most natural way is break up this box. In this simple example, the box represents a nucleus, which is made by particles that we want study. A natural solution is accelerate the two nuclei to relativistic velocities and collide them.

In an event, nuclei are imparted with a huge amount of kinetic energy, and when they collide, this energy is used to break up the nuclei [3], making possible to produce a new state of the matter, which basically consists of a "soup" of quarks and gluons. With the increasing temperature (heating) and/or the increasing baryon density (compression), a phase transition may occur to the state where ordinary hadrons do not exist anymore, and quarks and gluons themselves are the appropriate degrees of freedom. This phase transition is called QuarkGluon Plasma (QGP) [4-19].

This makes it possible to study the many-body properties of deconfined matter [20], determine properties of the QGP, and understand the initial stages from experimental data.

A system created by a collision of nuclei generates a large number of particles, and consequently, it is complicated to use only QCD to completely study the system. This work uses an effective hybrid model that combines a fluid dynamical description with particle description.

\footnotetext{
${ }^{1}$ Part of Quantum Field Theory (QFT) that deals with the strong (nuclear) interaction.
} 
In order to describe this hybrid model, it is necessary to understand the evolution of a collision system. During a collision between two heavy nuclei, there are some stages of evolution that can be shown in Figure 1.1.

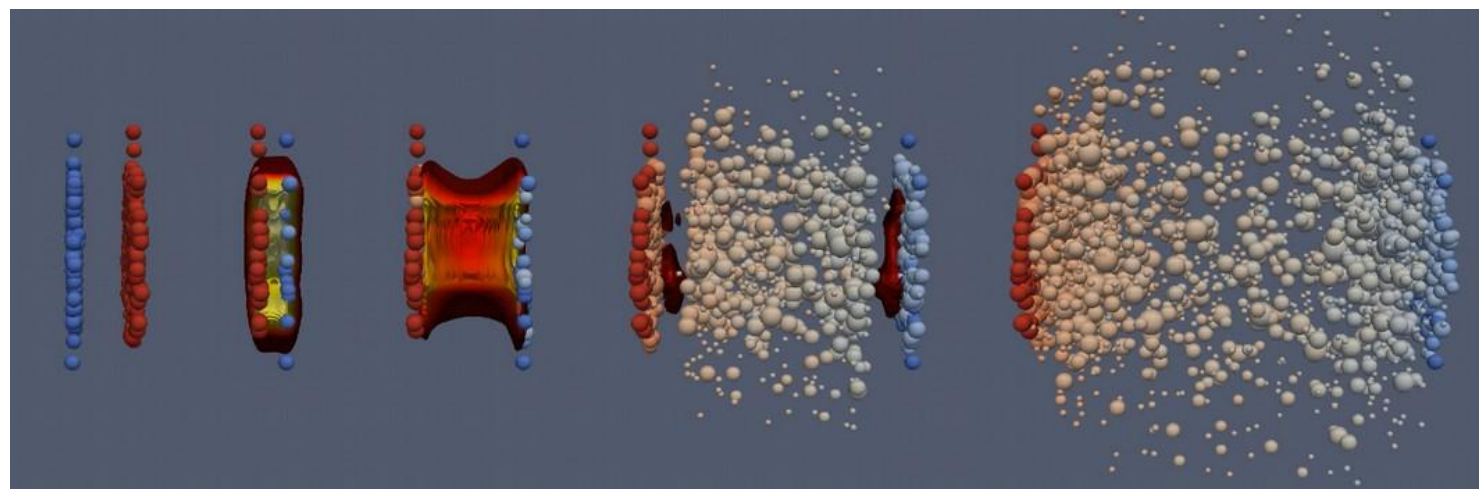

FIGURE 1.1: The time evolution of a typical heavy ion collision from MADAI collaboration, Hannah Petersen and Jonah Bernhard.

Below, all these stages are detailed respectively.

- Before collision: At this stage, the two nuclei are moving close to the speed of light which causes Lorentz contractions, which is why both are deformed at the figure. Since we have $\sim 260$ nucleons per ion, they will eventually interact.

- Early stage dynamics: After the nuclei collide, a "soup" of QCD matter is produced. However, the system must first evolve into a state that can be described with thermodynamic relations. A sophisticated way to describe this early time evolution is using the Color Glass Condensate (CGC)[21]. The collision system described by CGC leads to the glasma formation [14, 15] before, eventually, the QGP.

- Quark-gluon plasma: The thermalization of the glasma results in a strongly coupled quark-gluon plasma. Although the thermalizaion is not completely understood yet, we know that it is fast $\left(\tau_{\text {therm. }} \sim 1 \mathrm{fm}\right)$, and the initial temperature of the thermalized QGP is about $T \sim 400 \mathrm{MeV}$. In this way, the initial conditions for the hydrodynamic evolution of the QGP is provided by the matching of the initial energy-momentum tensor,

$$
T_{\text {initial }}^{\mu \nu}\left(\tau_{\text {therm. }}\right)=T_{\text {hydro }}^{\mu \nu}\left(\tau_{\text {therm. }}\right),
$$

where the term "initial" refers to some model (e.g. CGC) used to describe the early stages dynamics. However, the QGP phase is the focus in this work. This phase can be described by relativistic hydrodynamics and its existence was announced by Relativistic Heavy Ion Collider (RHIC) in 2004 [16, 18, 22, 23].

- Hadronization: As the medium expands and becomes more dilute, the stronglycoupled QGP begins to reconfine into a gas of hadrons [24-27]. Concomitant with its fast expansion, the QGP cools down and once it achieves the transition temperature, we have the formation of the hadrons, that begins at the edge of the plasma and 
usually ends in the central regions. Eventually, this hadron gas will achieve a temperature such that all the inelastic collisions stop, which is denoted as being the chemical freeze-out, since the species of hadron are maintained after this threshold temperature. As the temperature keeps decreasing, one has the kinetic freeze-out, wherein the elastic interactions stop (the gas does not interact anymore) and the momentum distribution is frozen.

- After hadronization: The gas of hadrons the hydrodynamic evolution still undergoes a complex dynamic. Unstable hadrons decay, and hadrons undergo elastic and inelastic collisions that change the momentum distribution and species composition of the hadron gas. Once all unstable hadrons have decayed into stable ones and once the hadron gas is too dilute for even hadronic interactions to occur, hadrons continue their way unhindered toward the detectors.

In general, the matter produced in a collision between atomic nuclei is definitively a system of interacting quarks and gluons, and the study of heavy ion collisions is a field interested in more precisely determine properties of the QGP [1].

Next chapter explains a model that describes the evolution of heavy ion collisions. This description is a hydrid model, which is based on fluid and particle description.

The experimental evidence for the existence of the QGP in heavy ion collisions could be related by elliptic flow that is characteristic of the collective behavior, which will be discussed in section 1.2. In the next section, we discuss about important issues regarding the viscosity of the QGP.

\subsection{The viscosity of the QGP}

One of the most important striking features of the QGP is that its time evolution can be modeled with relativistic fluid dynamics. Early studies are concentrated on ideal fluid [28], but the realization that hadronic data from relativistic heavy ion collisions could be used to extract the transport coefficients of QCD, in particular the shear viscosity to entropy density ratio $(\eta / s)$.

To understand the connection between the QGP and its viscosity, it is necessary to analyze some details about the geometry of the collision. In Figure 1.2, we have a schematic collision, where what is measured is the particle distribution in momentum space that is decomposed in terms of Fourier coefficients, such as

$$
E \frac{d N}{d^{3} p}=\frac{1}{2 \pi} \frac{d N}{p_{T} d p_{T} d Y} \sum_{n=-\infty}^{\infty} V_{n} e^{-i n \varphi}
$$

where $E$ is the particle's energy, $p_{T}$ is the transverse momentum, $\varphi$ is the azimuthal angle in momentum space, and $Y$ is the rapidity (more details about rapidity will be available in section 1.6). The $V_{n}$ is the Fourier coefficient associated with the respective mode, with the first having specific names like $V_{1}$ is the direct flow, $V_{2}$ is the elliptic flow, $V_{3}$ is the triangular flow, and so on. 


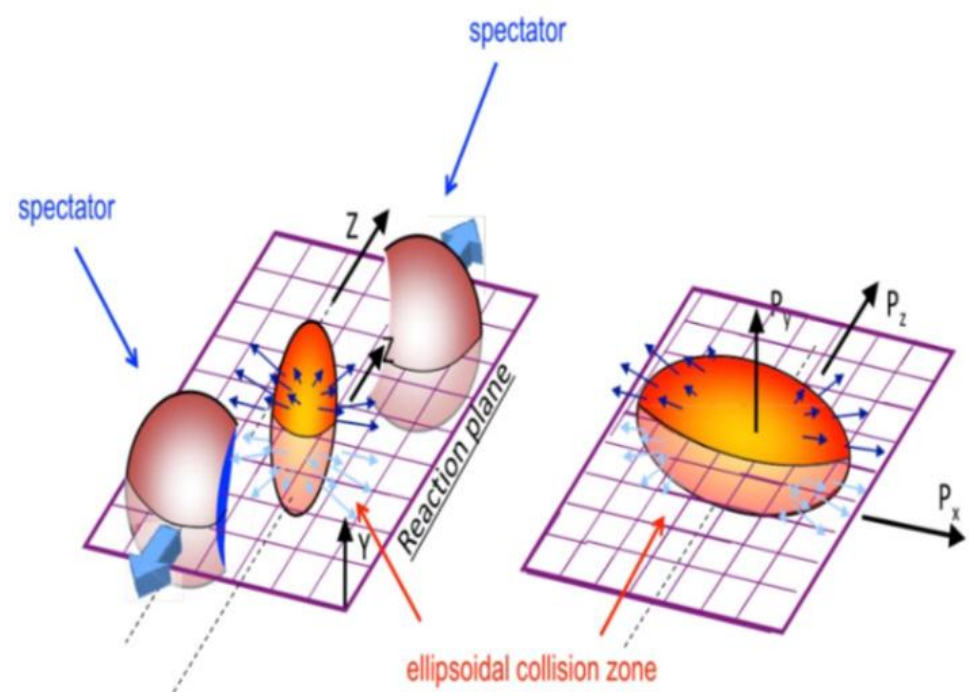

FIGURE 1.2: A typical heavy ion collision. Figure adapted from [29].

When the QGP is formed in a typical heavy ion collision, it has initially an ellipsoidal shape. As time goes by, this formed ellipsoid will expand, faster in the perpendicular direction of the collision (notice the momentum anisotropy on the left of the Figure 1.2), generating the elliptic flow. We can formally represent the momentum asymmetry using the eccentricity $\epsilon^{p}$ that can be written as

$$
\epsilon^{p}=\frac{\left\langle T^{x x}-T^{y y}\right\rangle}{\left\langle T^{x x}+T^{y y}\right\rangle}
$$

where $T^{x x}$ and $T^{y y}$ are the components of the stress tensor, with $\langle\ldots\rangle$ meaning that we are averaging it on the reaction plane. Intuitively, we can understand the elliptic flow as being originated from the gradient pressure of the QGP formed in the collision, with the large elliptic flow indicating that the partons of the QGP are interacting strongly with small shear viscosity to entropy density (momentum diffusion).

The question of whether relativistic hydrodynamics can describe elliptic flow satisfactorily is shown in Figure 1.3, which shows good agreement of the hydrodynamic model with the experimental data. Notice that, from the data analysis, we have a very small shear viscosity $(\eta / s=0.2)$ [30]. Therefore, there are reasons to believe that the QGP formed in these heavy ion collisions is strongly coupled, which characterizes the hydrodynamical behavior.

\subsection{Initial condition to final state mapping}

In a usual hydrodynamic simulation, the distribution of energy-momentum tensor $\left(T^{\mu \nu}\right)$ when the hydrodynamics become valid completely determine the final result, which is the 

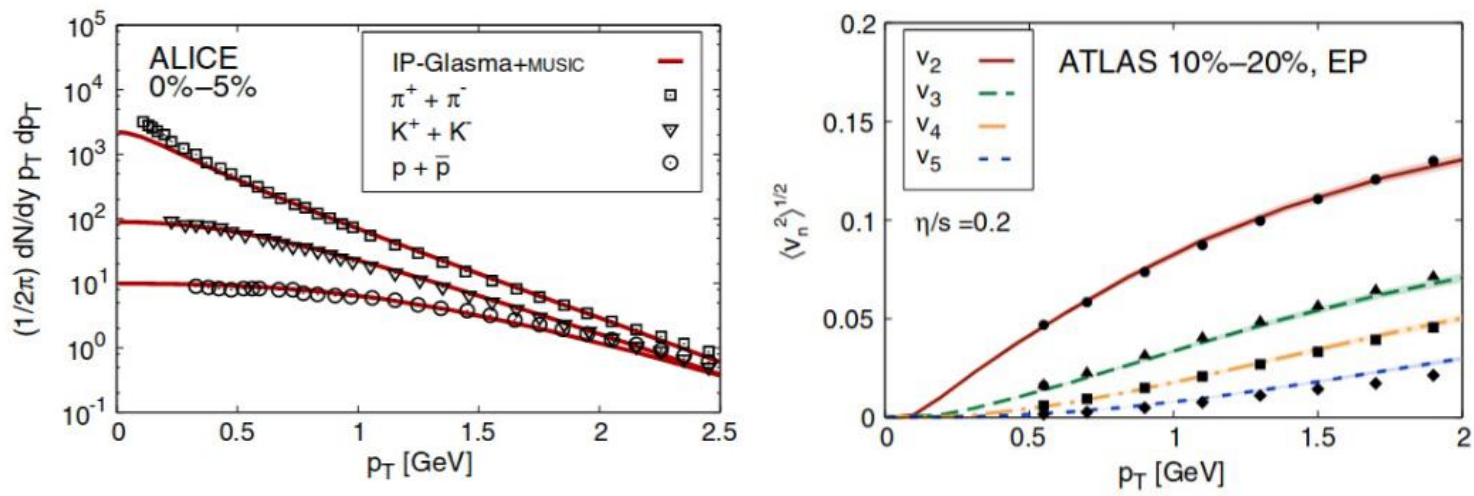

FIGURE 1.3: In (left), we have the success of the hydrodynamic modelling of the QGP (the red line crossing the experimental dots). The IP+Glasma is the initial condition model, whilst MUSIC is the hydrodynamic code that models the space-time evolution of the QGP. In the (rigth), we have the experimental coefficients $V_{n}$ are in good agreement with the theoretical model for $\eta / s=$

0.2. Figure adapted from [30]

final particle distribution $\left(E \frac{d N}{d^{3} p}\right)$. Then we have

$$
\left.\left.T^{\mu \nu}\right|_{\text {Initial }} \rightarrow E \frac{d N}{d^{3} p}\right|_{\text {Final }} .
$$

The final state of a heavy ion collision system can be characterized by the quantity $V_{n}$ (linked with the final anisotropic flow), and similarly, the initial condition can also be characterized by the quantity $\epsilon_{n}$ (linked with the spatial anisotropies in the initial system density).

The relationship between initial condition until the final state is a complicated process that can be represented by relatively simple relations, such as

$$
V_{n}=\kappa_{n} \epsilon_{n}
$$

where the anisotropic flow can be proportional to initial spatial anisotropies, it happens because the asymmetry of the system is conserved.

Relations such (1.5) identify the relevant properties of a initial stage and separate them from the effects of subsequent evolution $\left(\kappa_{n}\right)$. And through these simple relations, it is possible to determine the relevant information about the final state (after hadronization). These relations are derived from an expansion that will be explained at Equation 3.23, where its first term corresponds to $\kappa_{n} \epsilon_{n}$. Although there are other terms, this work is interested to consider only the first one in order to start since the simple case. In other words, final state $\left(V_{n}\right)$ is well understood as a hydrodynamic response to the initial condition profile $\left(\epsilon_{n}\right)$. In hydrodynamics, $V_{n}$ is typically a functional of the initial density profile [31,32]. With this knowledge, it is possible to put constraints on the initial stages with the initial stages from experimental data, as well as to determine properties of the QGP. 
The initial condition consists of the energy-momentum tensor at some early time. It is currently believed that the distribution of energy density in the transverse plane is the most important quantity driving the evolution of the system compared to other aspects of initial conditions such as the distribution of momentum density or stress tensor.

Under this assumption, the dependence of final observables on the initial energy density has been studied [33-36], and numerous results have been obtained that constrain specific properties of the initial state as well as the QGP medium. It is not known how important are the effects that are neglected in the approximation, some of these may indeed be unimportant. However, some may be quite significant particularly for smaller collision systems as protonnucleus and proton-proton, which are currently of great interest in the field.

In order to write quantities that represent initial conditions, a systematic expansion of the initial energy-momentum tensor that orders features in terms of their importance to subsequent hydrodynamic evolution was derived. Observables that characterizes the final stage are naturally separated into orthogonal modes with respect to azimuthal rotations. Thus, the energy density must be decomposed into modes with definite properties, so these quantities with the correct rotational symmetry can be constructed for each observable. The observables are entirely in momentum space because only the momentum of each outgoing particle can be measured, not it is spatial position when it is emitted (at least not directly). As such, one must also construct translation-invariant quantities. And finally, and crucially, the ansatz for hydrodynamics which assumes a large separation between microscopic and macroscopic scales, is only sensitive to long-length properties of the initial energy density distribution. All these properties are naturally obtained from a cumulant expansion of a Fourier transform of the energy density profile that was proposed by Teaney and Yan [32].

In order to include effects stemming from other components of the initial energy-momentum tensor such as the momentum density and stress tensor, a hydrodynamic framework in an nontrivial ansatz that is able to predict the final observables with more precision was constructed. Then each new contribution from initial conditions was tested numerically in order to check whether the ansatz could include these new effects. After this, simulations using initial conditions were made in order to demonstrate the efficacy of framework.

\subsection{Participants, spectators, and impact parameter}

In this field, it is important to define some technical terms, then as explained in [1], in heavyion collisions, simple geometric concepts are often used. For example, one separates so called participants from spectators, see Figure 1.4. If we assume that all nucleons propagate along parallel, straight line trajectories, then the nucleons which do not meet any other nucleons on their way are called spectators. Other nucleons which interact with each other are called participants.

A 2-D vector connecting the centers of the collision nuclei in the plane transverse to the nucleon trajectories is called impact vector, and its length is the impact parameter. 


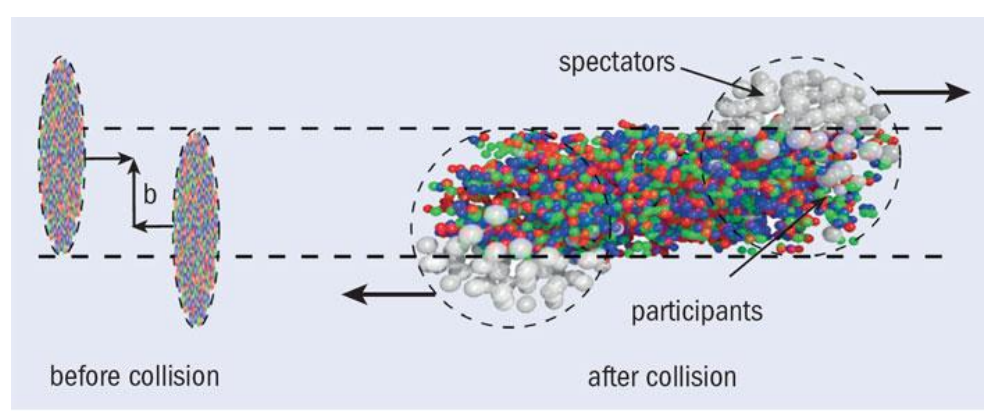

FIGURE 1.4: Impacter parameter, spectors and participants presented in Ref. [37].

The class of central collision ${ }^{2}$ corresponds to the zero impact parameter.

\subsection{Centrality}

So far we have interpreted the most central collisions as those corresponding to the smallest values of the impact parameter. In this section, we are going to introduce the quantitative measure of the centrality and relate it directly to the impact parameter. In experiments with heavy ions, centrality is estimated as the percentile of events with the measured multiplicity (as registered in detectors), or with the largest number of participants.

A good example to understand this concept different classes of centrality. Class $0-10 \%$ corresponds to the most central collisions characterized by the smallest values the impact parameter (compared with the other classes) and the largest values of the participating nucleons than $20-30 \%$.

In class of $0-10 \%$ of centrality also the largest multiplicities or entropy density (used in this work) of the produced hadrons are obtained. With increasing centrality, the number of the participants is reduced, and already class $20-30 \%$ the mean number of the participants is smaller. Note that the large centrality corresponds to a large value of the impact parameter and, hence, to a peripheral rather than to a central collision [38].

\subsection{Rapidity and pseudorapidity}

The component of a three-vector $\mathbf{A}$ parallel to $z$-axis is usually denoted by $\mathbf{A}_{s}$, and the transverse component is $\mathbf{A}_{T}=\mathbf{A}-\mathbf{A}_{s}$. [1,38], since we deal with relativistic energies, it is useful to use the rapidity instead of the standard velocity

$$
Y=\frac{1}{2} \ln \left(\frac{E+p_{s}}{E-p_{s}}\right)=\tanh ^{-1} \frac{p_{s}}{E}=\tanh ^{-1} v_{s}
$$

\footnotetext{
${ }^{2}$ In practice one considers a group of events which are characterized by the smallest values of the impact parameter. The concept of centrality is discussed more in section 1.5.
} 
where $E$ is the energy of a particle, such that $E=\sqrt{m^{2}+\mathbf{p}^{2}}$, and $v_{s}=p_{s} / E$ is the longitudinal component of the velocity.

Rapidity is additive under Lorentz boosts along the $z$-axis. This means that the difference $d Y$ does not change under Lorentz boosts along the collision axis. A boost-invariant system is independent of rapidity.

Experimentalists distinguish between rapidity and pseudorapidity. The latter is defined as

$$
\eta=\frac{1}{2} \ln \left(\frac{|\mathbf{p}|+p_{s}}{|\mathbf{p}|-p_{s}}\right)=-\ln \left(\tan \frac{\theta}{2}\right),
$$

where $\theta$ is the scattering angle. Pseudorapidity is easier to measure than rapidity (it is just a measure of the angle at which a particle has been emitted). To measure rapidity one has to identify the particle. Since at large energies $E \approx|\mathbf{p}|$ one is often tempted to assume that $d N / d Y \approx d N / d \eta$. In practice, this approximation is poor, especially in the region where rapidity is close to zero.

In theoretical calculations one usually uses the space-time rapidity

$$
\begin{gathered}
\eta_{s}=\frac{1}{2} \ln \left(\frac{t+z}{t-z}\right) . \\
\tau=\sqrt{t^{2}-z^{2}},
\end{gathered}
$$

where $\tau$ is the proper time. Lines of constant $\tau$ and constant $\eta_{s}$ are represented in Figure 1.5. In the neighbourhood of $z=0$, one has $\tau \simeq t$ and $\eta_{s} \simeq z / t$. We then use the property of boost-invariance mentioned above: any value of $z$ with $|z|<t$ can be brought to $z=0$ by means of a homogeneous Lorentz boost in the $z$ direction.

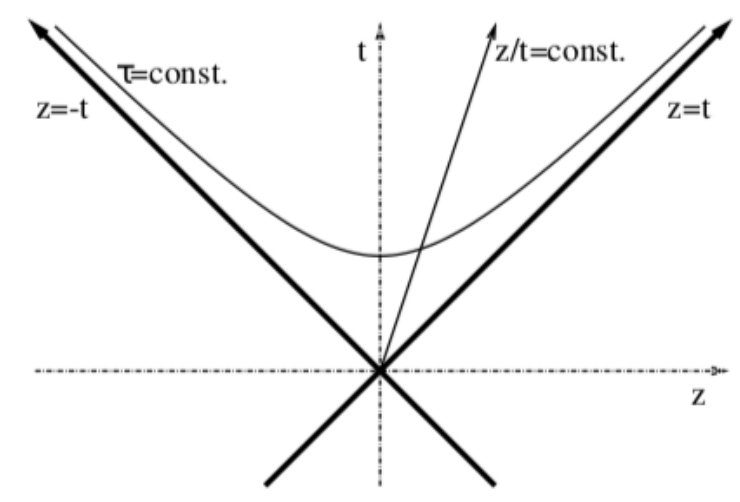

FIGURE 1.5: Schematic representation of a nucleus-nucleus collision in the $(z, t)$ plane. The thick lines are the trajectories of the colliding nuclei, which are moving nearly at the velocity of light. The lines of constant $z / t$ are also lines of constant $\eta_{s}$ made at Ref. [39]. 


\section{Chapter 2}

\section{Model description}

Our study is based on a hybrid model which combines viscous fluid dynamical description of the QGP stage with a realistic kinetic simulation of the hadronic stage [40-45]. Relativistic hydrodynamics is the most relevant framework to understand the transport properties of the QGP since it directly connects the collective flow developed during the QGP stage with its equation of state. It is based on the key assumption of local thermalization. Since this assumption breaks down during both the very anisotropic initial matter formation stage and the dilute late hadronic rescattering stage, the hydrodynamic framework can be applied at best only during the intermediate period. To describe the breakdown of the hydrodynamic description during the late hadronic stage due to expansion and dilution of the matter, one may have two options: one can either impose a sudden transition from thermalized matter to non-interacting or free-streaming hadrons through the Cooper-Frye prescription [46], making a transition from a macroscopic hydrodynamic description to a microscopic kinetic description at a switching temperature $T_{S W}$.

In this work, we use the MUSIC [47-49] 3+1D hydrodynamic code. All initial conditions used are boost-invariant, more details about the rapidity dependence are available in [50, 51]. The equation of state used in this work is that of parametrization "s95p-v1" from [52], obtained from interpolating between lattice data and hadron resonance.

\subsection{Relativistic hydrodynamics}

Hydrodynamics is used to describe the space-time evolution of the energy-momentum tensor $T^{\mu \nu}$ of the strongly-coupled quark-gluon plasma. This makes it possible to learn about certain general many-body properties of QCD without necessarily tracking the complicated dynamics of every quark, gluon and hadron in the system. Such properties of QCD include the equation of state and the viscosities of QCD.

As explained in [39],

Standard Thermodynamics is about a system in global thermodynamic equilibrium. This means that intensive parameters $(\mathcal{P}, T, \mu)$ are constant throughout the volume, and also that the system is globally at rest, which means that its total momentum is 0 . In this section, pressure and temperature vary with space and time, and which are not at rest, such as indian atmosphere during monsoon. We however request that the system is in local thermodynamic equilibrium, which 
means that pressure and temperature are varying so slowly that for any point, one can assume thermodynamic equilibrium in some neighbourhood about that point. Here, 'neighbourhood' has the same meaning as in mathematics, and there is no prescription as to the actual size of this neighbourhood, or 'fluid element'. There is, however, a general condition for local thermodynamic equilibrium to apply, which is that the mean free path of a particle between two collisions is much smaller than all the characteristic dimensions of the system. (Ollitrault, 2008)

The fluid equations derived under the assumption of local thermodynamic equilibrium are called ideal-fluid equations.

In hydrodynamics, the energy-momentum tensor $T^{\mu \nu}$ is a contravariant tensor, where each value of $\nu$ corresponds to a component of the four-momentum, and $\mu$ to a four-component of energy flux. Specifically,

- $T^{\tau \tau}$ is the energy density

- $T^{\tau j}$ is the density of $j^{\text {th }}$ component of momentum, with $j \in\{1,2,3\}$

- $T^{i \tau}$ is the energy flux along axis $i$

- $T^{i j}$ is the flux along axis $i$ of the $j^{t h}$ component of momentum.

It is important to note that $T^{\mu \nu}$ is symmetric, it means that the momentum density $T^{\tau i}$ and the energy flux $T^{i \tau}$ are equal.

The momentum flux $T^{i j}$ is usually called the stress tensor.

\subsubsection{Ideal hydrodynamics}

Starting with the most simple case as [39], this subsection deals with systems whose pressure and temperature vary with space and time, and which are not at rest.

The rest frame of a fluid element is the Lorentz frame in which its momentum vanishes.

The velocity $\vec{v}$ of a fluid element is defined as the velocity of the rest frame of this fluid element with respect to the laboratory frame. The four-velocity $u^{\mu}$ is defined by

$$
\begin{aligned}
u^{0} & =\frac{1}{\sqrt{1-\vec{v}^{2}}}, \\
\vec{u} & =\frac{\vec{v}}{\sqrt{1-\vec{v}^{2}}},
\end{aligned}
$$

where we have chosen a unit system where $c=1 . u^{0}$ is the Lorentz contraction factor. The four-velocity transforms as a four-vector under Lorentz transformations. The square of a four-vector is a Lorentz scalar, and we indeed obtain

$$
u^{\mu} u_{\nu}=u^{0} u_{0}-\vec{u}^{2}=1 .
$$


The energy-momentum in the fluid rest frame is thus

$$
T_{(0)}=\left(\begin{array}{cccc}
\epsilon & 0 & 0 & 0 \\
0 & \mathcal{P} & 0 & 0 \\
0 & 0 & \mathcal{P} & 0 \\
0 & 0 & 0 & \mathcal{P}
\end{array}\right) .
$$

In order to obtain the energy-momentum tensor in a moving frame, one does a Lorentz transformation.

Under a Lorentz transformation, the contravariant tensor $T^{\mu \nu}$ transforms to

$$
T^{\mu \nu}=\Lambda_{\alpha}^{\mu} \Lambda_{\beta}^{\nu} T_{(0)}^{\alpha \beta},
$$

which can be written as a multiplication of $(4 \times 4)$ matrices

$$
T=\Lambda T_{(0)} \Lambda^{T}
$$

where $\Lambda^{T}$ denotes the transpose of $\Lambda$, where that $\Lambda$ is symmetric, $\Lambda^{T}=\Lambda$.

The energy-momentum tensor for an arbitrary fluid velocity is

$$
T^{\mu \nu}=(\epsilon+\mathcal{P}) u^{\mu} u^{\nu}-\mathcal{P} g^{\mu \nu},
$$

where $g^{\mu \nu} \equiv \operatorname{diag}(1,-1,-1,-1)$ is the Minkovski metric tensor. One easily checks that this equation reduces to Eq. (2.3) in the rest frame of the fluid, where $u^{\mu}=(1,0,0,0)$. In addition, both sides of Eq. (2.6) are contravariant tensors, which means that they transform identically under Lorentz transformations. Since they are identical in one frame, they are identical in all frames, which proves the validity of Eq. (2.6).

The conservation equations of energy and momentum are

$$
\partial_{\mu} T^{\mu \nu}=0
$$

Eqs. (2.6) and (2.7) are the equations of ideal-fluid relativistic hydrodynamics.

\subsubsection{Viscous hydrodynamics}

And in the case of viscous hydrodynamics, the energy-momentum tensor has the form

$$
T^{\mu \nu}=T_{\text {ideal }}^{\mu \nu}+\Pi^{\mu \nu}
$$

where $T_{\text {ideal }}^{\mu \nu}$ is the fluid-perfect part given by Eq. (2.6) and $\Pi^{\mu \nu}$ is the viscous tensor and describes dissipation $[53,54]$

$$
\begin{aligned}
& \Pi^{\mu \nu}=\pi^{\mu \nu}+\Pi \Delta^{\mu \nu}, \\
& \Delta^{\mu \nu}=g^{\mu \nu}-u^{\mu} u^{\nu} .
\end{aligned}
$$

Here $\pi^{\mu \nu}$ is the shear tensor and $\Pi$ describes the viscous the viscous bulk pressure. The equations of hydrodynamics follow from the conservation laws for energy and momentum, 
and from the requirement that the entropy production is positive. These conditions determine the form of equations to be satisfied by dissipative terms $\pi^{\mu \nu}$ and $\Pi$.

From the formal point of view, the inclusion of the dissipative terms in (2.9) follows from the gradient expansion around the local equilibrium. In the first order in gradients one finds the Navier-Stokes expressions

$$
\begin{aligned}
\pi^{\mu \nu} & =\eta \nabla^{<\mu} u^{\nu>}, \\
\Pi & =\varsigma \partial_{\alpha} u^{\alpha},
\end{aligned}
$$

where the angle brackets project out the traceless symmetric part (the symmetric part denoted by round brackets)

$$
\begin{aligned}
\nabla^{<\mu} u^{\nu>} & =2 \nabla^{(\mu} u^{\nu)}-\frac{2}{3} \Delta^{\mu \nu} \nabla_{\alpha} u^{\alpha}, \\
\nabla^{\alpha} & =\Delta^{\alpha \beta} \partial_{\beta} .
\end{aligned}
$$

The quantities $\eta$ and $\varsigma$ in (2.9) are the shear and bulk viscosity, respectively. Unfortunately, the relativistic fluid dynamics based on the Navier-Stokes prescription suffers from problems connected with the acausal transmission of signals. This is why the second-order theory had been developed by Israel and Stewart [55]. Within the second-order theory, the shear tensor $\pi^{\mu \nu}$ and the bulk pressure $\Pi$ satisfy non-trivial dynamic equations. They are not any longer expressed by simple formulas such as (2.10). Moreover, the second-order theory requires that higher-order kinetic coefficients should be introduced.

At the moment, the formalism developed by Israel and Stewart is the most popular version of the dissipative hydrodynamics used to describe heavy ion collisions. Usually only the shear viscosity is included in such calculations. There are, however, suggestions that the bulk viscosity may also play an important role [56]. More importantly, the second-order formalism may lead to unphysical behavior to the early stages of the collisions or at the edges of the produced system. Such issues are discussed in the lectures by Michael Strickland [57] in the context of a new formulation of dissipative fluid dynamics.

\subsubsection{Before hydrodynamics}

In these work, we define the early stage of heavy ion collisions as everything that happens before the plasma can be described by hydrodynamics.

Hydrodynamics describes the evolution of the strongly-coupled quark-gluon plasma in space and time. It can describe this evolution if it is provided the state of the fluid on a given spacetime surface ${ }^{1}$.

Remember that, with second-order viscous hydrodynamics, the energy-momentum tensor $T^{\mu \nu}$ is decomposed into four independent hydrodynamic fields: the energy density $\epsilon(\vec{x})$, the fluid velocity $u^{\mu}(\vec{x})$, the shear stress tensor $\pi^{\mu \nu}(\vec{x})$, and the bulk pressure $\Pi(\vec{x})$

In almost every simulation of heavy ion collisions, the equations of hydrodynamics are initialized at a fixed value of $\tau=\tau_{0}$. That is, hydrodynamic initial conditions are provided

\footnotetext{
${ }^{1}$ That is, if hydrodynamics is taken as nothing more than a system of partial differential equations, boundary conditions or initial conditions must be provided before a numerical solution of the equations can be obtained.
} 
as $T^{\mu \nu}\left(\tau_{0}, \vec{x}\right)$. More typically the different fields are initialized separately: $\epsilon\left(\tau_{0}, \vec{x}\right), u^{\mu}\left(\tau_{0}, \vec{x}\right)$, $\pi^{\mu \nu}\left(\tau_{0}, \vec{x}\right)$ and $\Pi\left(\tau_{0}, \vec{x}\right)$ separately, here we can understand $\vec{x}$ as $\left(x, y, \eta_{s}\right)$. Note that these initial conditions represents a considerable amount of information. To understand how much of a challenge it is to determine $T^{\mu \nu}$, it is worth taking a step back and look at the initial moments of a heavy ion collisions.

The initial conditions of hydrodynamics hide a lot of the complexity of the early stage of heavy ion collisions. Between the moment that the nuclei collide and the time at which hydrodynamics become applicable, there is a complex dynamical evolution of the deconfined matter. Moreover the fact that there are fluctuations in heavy ion collisions implies that there is no single initial condition corresponding to two nuclei colliding. There is rather a family, or a distribution, of initial conditions.

\subsubsection{After hydrodynamics}

Hydrodynamics cannot describe a fluid of arbitrary low density, in particular if this fluid is expanding rapidly as the case in heavy ion collisions. Physically, this means that the hydrodynamics description of the plasma produced in heavy ion collisions must be stopped at some point, and that the matter being described with hydrodynamics must be converted into different degrees of freedom. This procedure can be called particlization, since the fluid is converted into particles. This conversion from fluid to particle is performed in simulations of heavy ion, using the Cooper-Frye prescription.

It is important not to confuse particlization/Cooper-Frye with hadronization and freezeout, which are some times used loosely to refer to particlization. Technically, hadronization refers to the reconfinement of the strongly-coupled quark-gluon plasma into hadrons. However, hadronization and particlization do not have to happen at the same time. Actually, Cooper-Frye requires that hadronization happens before particlization, and freeze-out refers to the moment when interactions among particles stop completely (or, in practice, when these interactions become negligible). In other words, The kinetic freeze-out is a transition from a strongly coupled system to a weakly coupled one. For a hadron gas, it implies that the gas is so dilute that the hadrons do not interact with each other anymore.

Freeze-out can also refer to two separate concepts: chemical freeze-out, when inelastic collisions stop, and kinetic freeze-out, when both inelastic and elastic collisions stop. When freeze-out is used alone, it usually refers to the kinetic freeze-out.

The Cooper-Frye formula will be discussed in next section 2.2. What is relevant to discuss here is the particlization criteria. The criteria that is used to stop the hydrodynamics and convert the fluid into hadrons. In almost all hydrodynamics simulations of heavy ion collisions, particlization is assumed to happen at a given temperature (or energy density, which is related to temperature through the equation of state). As the temperature of the hydrodynamic medium decreases, different parts of the medium reach the particlization temperature. Connecting all the points at which the local temperature of the medium cross the particlization temperature forms a 4D spacetime hypersurface. This hypersurface is complete once every point in the hydrodynamic medium is below this particlization criteria, and hydrodynamics can then be stopped. 


\subsection{Relativistic kinetic theory of gases}

This theory describes a gas as a large number of particles. The information about this system is comprised in a distribution of particles in the phase space which gives us the statistics of the gas. This quantity is called distribution function and can be written as

$$
f(x, p)=\frac{d N}{d^{3} x d^{3} p}
$$

where

$$
d N=f(x, p) d^{3} x d^{3} p
$$

denotes the number of particles in the phase space with volume $d^{3} x d^{3} p$.

Using this definition, it is possible to extract macroscopic quantities from Kinetic theory by taking averages (moments). For instance, we can determine quantities as particle density, particle flux and the 4-vector number current, respectively as

$$
\begin{aligned}
n(t, \vec{x}) & =\int d^{3} p f(x, p) \\
\vec{n}(x) & =\int d^{3} p \frac{\vec{p}}{E} f(x, p) \\
n^{\mu}=(n, \vec{n}) & \equiv \int d^{3} p p^{\mu} f(x, p),
\end{aligned}
$$

where the term $p^{\mu}$ is the four-momentum defined as $\left(p^{0}=E, \vec{p}\right)$.

The equilibrium distribution function for a classical dilute gas at rest is given by the well-known Boltzmann distribution. In other cases, for a quantum gas, we can have either the Fermi-Dirac distribution for fermions or the Bose-Einstein distribution for bosons. However, the study of heavy ion collisions deals with high-energies and requires the usage of the relativistic version of the Boltzamnn equation (more details available in [58]).

The relativistic generalization of the Boltzmann distribution in equilibrium [58] is

$$
f_{e q}(x, p)=\frac{1}{e^{\left(\frac{p^{\mu} u_{\mu}-\mu}{T}\right)}+K},
$$

where the term $\mu$ is the chemical potential, $T$ is the temperature, and in ideal gas the factor $K$ is 1 for bosons, -1 for fermions, and 0 for distinguishable particles. In other case, in local equilibrium, we have

$$
f(x, p) \rightarrow f_{e q}\left(t, \vec{x}, u^{\mu} p_{\mu}\right)=\frac{1}{e^{\left(\frac{p^{\mu} u_{\mu}(x)-\mu(x)}{T(x)}\right)} \pm 1},
$$

where $p^{\mu} u_{\mu}$ is the four-velocity (momentum). 
In near equilibrium [59] one expects the particle distribution function to be close to $f_{e q}$ such as

$$
f(x, p)=f_{e q}\left(x, u^{\mu} p_{\mu}\right)+\delta f(x, p),
$$

that has this form because usually we consider small departures from equilibrium.

It is possible to establish a relation between $T^{\mu \nu}(t, \vec{x})$ on a freeze-out surface with the distribution function such as

$$
\begin{aligned}
T^{\mu \nu}(t, \vec{x}) & =\int \frac{d^{3} p}{E} p^{\mu} p^{\nu} f(x, p) \\
& =\int \frac{d^{3} p}{E} p^{\mu} p^{\nu} f_{e q}+\int \frac{d^{3} p}{E} p^{\mu} p^{\nu} \delta f(x, p)
\end{aligned}
$$

where as shown in previous section, this energy-momentum tensor can be written as

$$
\begin{aligned}
T^{\mu \nu} & =T_{\text {ideal }}^{\mu \nu}+\Pi^{\mu \nu} \\
& =(\epsilon+\mathcal{P}) u^{\mu} u^{\nu}-\mathcal{P} g^{\mu \nu}
\end{aligned}
$$

where

$$
T_{i d e a l}^{\mu \nu}=\int \frac{d^{3} p}{E} p^{\mu} p^{\nu} f_{e q}
$$

and

$$
\Pi^{\mu \nu}=\int \frac{d^{3} p}{E} p^{\mu} p^{\nu} \delta f
$$

The distribution of particle in momentum space can be given such as

$$
\begin{aligned}
\frac{d N}{d^{3} p} & =\int d^{3} x \overbrace{f(x, p)}^{f\left(t=t_{s}, \vec{x}, p\right)} \\
E \frac{d N}{d^{3} p} & =\int d^{3} x E f\left(t_{s}, \vec{x}, p\right),
\end{aligned}
$$

where we can switch at constant time like $t_{s}$.

Hybrid model transitions from hydrodynamic field equations to microscopic transport at a sudden switching temperature $T_{S W}$ at which the hydrodynamic energy-momentum tensor is particlized using Cooper-Frye freeze-out prescription, $T^{\mu \nu}$ is converted to hadrons using the Cooper-Frye formula [60], that can be written as

$$
E \frac{d N}{d^{3} p}=\int_{\sigma} d^{3} \sigma_{\mu} p^{\mu} f_{i}\left(t_{s}, \vec{x}, p\right)
$$

where $f_{i}$ is the distribution function of particle species $i$, and $d^{3} \sigma_{\mu}$ characterizes an element of the isothermal freeze-put hypersurface defined by $T_{S W}$. 


\subsection{Final hadron dynamics}

Particlization describes the conversion of the fluid to hadronic degrees of freedom. In practice, in simulations, this means that the momentum distribution for each species of hadrons is tabulated from the particlization hypersurface.

Physically particlization is the transition from the strongly-coupled quark-gluon plasma reconfined to a gas of hadrons. These hadrons continue to interact until it is too dilute. Moreover most of the hadrons in this gas are unstable and decay into stable and unstable hadrons. These decay continue until only hadrons that are stable remain.

In the case of this work, the hydrodynamic code does not include a simulation of hadronic interaction, including such interaction requires the addition of hadronic transport models such as UrQMD afterburner (Ultra relativistic Quantum Molecular Dynamics) [61-63]. 


\section{Chapter 3}

\section{Mapping the hydrodynamic response}

\subsection{Characterizing the initial stage of a heavy-ion collision}

We assume that the evolution of the collision system is entirely determined by an initial condition that consists of the energy-momentum tensor at some early time $T^{\mu \nu}\left(\tau=\tau_{0}, \vec{x}\right)$. This is true in particular for standard hydrodynamic calculations.

Under this assumption, the final particle distribution in a given event $E \frac{d N}{d^{3} p}$ is a deterministic functional of the initial energy-momentum profile in the transverse plane, as well as the currents, in that event,

$$
E \frac{d N}{d^{3} p}(\vec{p})=\mathcal{F}\left[T^{\mu \nu}\left(\tau_{0}, \vec{x}\right)\right]
$$

The azimuthal dependence of the particle distribution is of particular interest, and can be usefully organized as a set of Fourier coefficients in the azimuthal angle $\varphi$ of the outgoing particle momentum [64]. Here it will be convenient to write it as a complex Fourier series

$$
E \frac{d N}{d^{3} p}(\vec{p}) \equiv \frac{1}{2 \pi} \frac{d N}{d \eta p_{T} d p_{T}} \sum_{n=-\infty}^{\infty} V_{n}\left(p_{T}, \eta\right) e^{-i n \varphi},
$$

not least because it is observed that the entire angular distribution can typically be characterized by only a few of the lowest harmonics $V_{n}$.

In this work we focus on momentum-integrated observables. Integrating over $p_{T}$ and $\eta$, Eq. (3.2) becomes

$$
\frac{d N}{d \varphi}=\frac{N}{2 \pi} \sum_{n=-\infty}^{\infty} V_{n} e^{-i n \varphi} .
$$

Here we write the harmonic decomposition as a complex Fourier series, where the standard $v_{n}$ and $\Psi_{n}$ are the magnitude and phase of the complex number $V_{n}$,

$$
V_{n}=v_{n} e^{i n \Psi_{n}}
$$

Harmonic flows coefficients $V_{n}$ contain information about the final particle distribution $E \frac{d N}{d^{3} p}$ and hence, $V_{n}$ can also be written as

$$
V_{n}=\mathcal{F}\left[T^{\mu \nu}(\vec{x})\right]
$$


Each collision event will present a different initial $T^{\mu \nu}$ which will result in a momentum distribution given by this functional (which itself is the same in every event).

Further, we assume that the structure of $T^{\mu \nu}(\vec{x})$ at long length scales is more important than small scale structure for the subsequent evolution of the system, such that we can express the final momentum distribution of various particles as a perturbative expansion in terms of cumulants of a Fourier transform. The lowest cumulants represent the smallest Fourier momenta, and therefore the largest structures. Since small-scale structure is assumed to be less important, higher terms in the series are less important, and the series can be truncated.
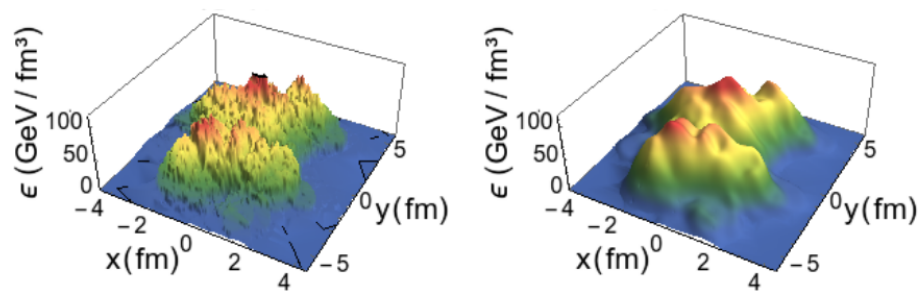

FIGURE 3.1: Examples of initial energy density at midrapidity for a $\mathrm{Pb}+\mathrm{Pb}$ collision, where its structure on the (left) has a more granular distribution with local details and on the (right) has a smoother aspect. Figure from [65].

As said in [65],

the influence of small scale structure within initial condition on the final observables, we systematically filter a variety of initial conditions using the separation of scales such that global large scale structure (e.g. eccentricities) are not significantly changed but small scale structure is filtered out. Using relativistic hydrodynamics, it was find that a variety of integrated $V_{n}$ flow observables and $V_{n}$ distributions remain insensitive to small scale structure. Then, at the same reference it was identified that these two initial conditions have a similar global structure and consequently a similar final state. (Gandim, 2019)

In the case, initial condition, for example, an energy density $\epsilon(\vec{x})$ is assumed that contribute to the relevant cumulant expansion was proposed by Teaney and Yan [32]. It was found that such a perturbative expansion works exceptionally well to describe the results of hydrodynamic simulations.

In the next chapter, we extend this idea to incorporate the contributions of the initial momentum density $T^{\tau i}(\vec{x})$ and initial stress tensor $T^{i j}(\vec{x})$ to develop a general framework that is valid even when components beyond the energy density are present, and which may even be valid for systems that are not described by hydrodynamics. The only requirement is some sort of separation of scales, such that large-scale structures are more important than small-scale structures. 
Now about the final stage, the flow coefficients $V_{n}$ are defined with respect to azimuthal rotations. In order to characterize the functional in Eq. (3.5), we must decompose the initial energy-momentum tensor into modes with definite rotational properties.

We note that the flow coefficients have the following rotation. Under a rotation of the system such as $\phi \rightarrow \phi+\delta$, the complex coefficients $V_{n}$ change as

$$
V_{n} \rightarrow V_{n} e^{i n \delta}
$$

Note that any other properties and symmetries must also be taken into account. For example, the harmonic flows are translation-invariant and dimensionless.

\subsection{A cumulant expansion}

In this section, we start by reviewing the cumulant expansion for a generating function $\rho(\vec{x})$ that can be replaced by energy density for example. We are only interested in azimuthal rotation properties, so we only consider the dependence on transverse coordinates $\{x, y\}$, meaning that we have a system which is approximately symmetrically distributed into longitudinal dependence (boost-invariant). The cumulant decomposition can be done at a given spacetime rapidity, or with any particular average or moment of the rapidity distribution, and it can be studied separately what range of spacetime rapidities contribute to particle distributions at a given rapidity or pseudorapidity.

The natural way to identify large- and small-scale structures is to take a (2-D) Fourier transform

$$
\rho(\vec{k})=\frac{1}{2 \pi} \int d^{2} x \rho(\vec{x}) e^{i \vec{k} \cdot \vec{x}}
$$

Naively, small momenta $k$ are associated with large-scale structure, and large $k$ represents small scales. Thus, we assume that the Fourier-transformed density is sufficiently well behaved as to be decomposed in a Maclaurin series, expanding around $|\vec{k}|=0$. In order to identify quantities with the correct rotational properties, we switch from Cartesian to polar coordinates and expand the Maclaurin coefficients in a Fourier series with respect to the azimuthal angle $\phi_{k}$

$$
\rho(\vec{k})=\sum_{m=0}^{\infty} \sum_{n=-\infty}^{\infty} \rho_{n, m} k^{m} e^{-i n \phi_{k}} .
$$

We can express the general moment $\rho_{n, m}$ in terms of the original density $\rho(\vec{x})$ by inverting the transformations,

$$
\rho_{n, m}=\frac{i^{m}}{2^{m}\left(\frac{m+n}{2}\right) !\left(\frac{m-n}{2}\right) !} \int d^{2} x r^{m} e^{i n \phi} \rho(\vec{x}),
$$

where $\phi=\tan ^{-1}(y, x)$ is the coordinate azimuthal angle, and subject to the constraint that the only non-zero values are for $m \geq|n|$ and when the difference $(m-n)$ is an even number.

A problem with these moments, however, is that they depend on the choice of the origin of the coordinate system. Since the final particle distribution is translation-invariant, the 
moments $\rho_{n, m}$ are not appropriate for characterizing the harmonics $V_{n}$. Another way of saying this is that small $k$ does not, in fact, represent large-scale structure in general, but instead structure far from the chosen center of coordinates, which is not the same.

The correct quantities are instead the cumulants of the density [32]. That is, we reset the generating function as

$$
\rho(\vec{k}) \equiv e^{W(\vec{k})},
$$

and expand $W(\vec{k})$ in Maclaurin and Fourier series as the same way before,

$$
W(\vec{k})=\sum_{n=-\infty}^{\infty} \sum_{m=|n|}^{\infty} W_{n, m} k^{m} e^{-i n \phi_{k}} .
$$

One cannot write a general expression for the cumulants $W_{n, m}$, and they must instead be generated iteratively order-by-order. This is not a problem, since we want to truncate the series at a finite $m$.

The first few cumulants are

$$
\begin{gathered}
W_{0,0}=\ln [\rho(\vec{k}=\overrightarrow{0})] \\
W_{1,1}=\frac{i}{2}\left\langle r e^{i \phi}\right\rangle \\
W_{0,2}=\frac{1}{2} \frac{i^{2}}{2 !}\left[\left\langle r^{2}\right\rangle-\left|\left\langle r e^{i \phi}\right\rangle\right|^{2}\right] \\
W_{2,2}=\frac{1}{4} \frac{i^{2}}{2 !}\left[\left\langle r^{2} e^{i 2 \phi}\right\rangle-\left\langle r e^{i \phi}\right\rangle^{2}\right] \\
W_{1,3}=\frac{1}{8} \frac{i^{3}}{2 !}\left[\left\langle r^{3} e^{i \phi}\right\rangle-\left\langle r^{2} e^{i 2 \phi}\right\rangle\left\langle r e^{-i \phi}\right\rangle\right. \\
\left.-2\left\langle r^{2}\right\rangle\left\langle r e^{i \phi}\right\rangle+2\left\langle r e^{i \phi}\right\rangle^{2}\left\langle r e^{-i \phi}\right\rangle\right] \\
W_{3,3}=\frac{1}{8} \frac{i^{3}}{3 !}\left[\left\langle r^{3} e^{i 3 \phi}\right\rangle+\left\langle r e^{i \phi}\right\rangle\left(3\left\langle r^{2} e^{i 2 \phi}\right\rangle-2\left\langle r e^{i \phi}\right\rangle^{2}\right)\right],
\end{gathered}
$$

where the brackets represent a spatial average weighted by the density $\rho(\vec{x})$,

$$
\langle\ldots\rangle=\frac{\int d^{2} x \ldots \rho(\vec{x})}{\int d^{2} x \rho(\vec{x})} .
$$

The cumulants with negative $n$ are redundant, since they are trivially related to those with positive $n$.

Calculations with more detailed derivation are available in appendix A.

With this definition, all of the cumulants except $W_{1,1}$ are now translation-invariant, and truly represent large- or small-scale structure according to the value of $m$. 
We can show this explicitly. For any function $\rho(\vec{x})$, under a translation of the system such that $\rho(\vec{x}) \rightarrow \rho(\vec{x}+\vec{R})$,

$$
\begin{aligned}
\rho(\vec{x}) \rightarrow \rho^{\prime}(\vec{x}) & =\int d^{2} x \rho(\vec{x}+\vec{R}) e^{i \vec{k} \cdot \vec{x}} \\
& =\int d^{2} x \rho(\vec{x}) e^{i \vec{k} \cdot(\vec{x}-\vec{R})} \\
& =e^{i \vec{k} \cdot \vec{R}} \rho(\vec{k}),
\end{aligned}
$$

and

$$
\begin{aligned}
W(\vec{k})=\ln (\rho(\vec{k})) & \rightarrow \ln \left(e^{i \vec{k} \cdot \vec{R}} \rho(\vec{k})\right) \\
& =W(\vec{k})-i k x \cos \left(\phi_{k}-\phi\right) .
\end{aligned}
$$

So the $m=1$ cumulants obtain an additive constant, while all others are translationinvariant.

If one chooses the center of coordinates such that $\langle x\rangle=\langle y\rangle=0\left(W_{1,1}=0\right)$, then the lowest cumulant $W_{n, m}$ coincides with $\rho_{n, m}$. Because of this, the issue of translationinvariance is typically ignored (beyond the need to do event-by-event recentering). However, it is a symmetry of $V_{n}$ that must be respected, and it will be important to keep it in mind as we proceed.

The index $n$ represents the rotational property of each cumulant. Similar to $V_{n}$ of Eq. (3.6), in our complex notation a rotation of the system such as $\phi \rightarrow \phi+\delta$ changes the cumulants as

$$
W_{n, m} \rightarrow W_{n, m} e^{i n \delta}
$$

As we have the Eq. (3.5), $V_{n}$ can be written as a function of $W_{n, m}$ as

$$
V_{n}=f\left(W_{n, m}\right)
$$

In this case, one can write a power series expansion, ensuring that each term has the same rotational properties of $V_{n}$

$$
V_{n}=\sum_{m=n}^{m_{\max }} \kappa_{n, m} W_{n, m}+\sum_{l=1}^{m_{\max }} \sum_{m=l}^{m_{\max }} \sum_{m^{\prime}=|n-l|}^{m_{\max }} \kappa_{l, m, m^{\prime}} W_{l, m} W_{n-l, m^{\prime}}+O\left(W^{3}\right) .
$$

The sums at non-linear order should be understood to neglect cumulants with $n=0$, as only the $n \neq 0$ cumulants are assumed to be small, and the resulting terms would therefore be redundant with respect to terms at lower order.

By truncating the cumulant expansion at some finite $m_{\max }$ (representing some minimum desired length scale), we obtain a finite number of terms at each order in the power series, which itself can be truncated at a finite order (leading order: $V_{n}=\kappa_{n, n} \epsilon_{n, n}$ ).

With this definition the coefficients $\kappa_{n, m}$ must be dimensionful, since the $V_{n}$ are defined to be dimensionless. They may well depend on scales that present themselves in the initial energy-momentum tensor, which therefore change from one event to another. We want to 
have an explicit characterization of the dependence on the initial condition, with unknown dimensionless coefficients that characterize only the response function. At the same time, only rotation-invariant quantities are assumed to be small, and only $n \neq 0$ cumulants show up in the expansion, Eq. (3.23).

As a result, one must divide each of these dimensionful cumulants by a relevant scale in order to obtain a dimensionless predictor for $V_{n}$ which is our goal to figure out. Therefore, the standard eccentricities [66] are obtained by making the choice to divide each cumulant $W_{n, m}$ by $\left\langle r^{m}\right\rangle$, and this is the most common choice.

In order words, we can define

$$
\epsilon_{n, m} \equiv-\frac{\left\langle r^{m} e^{i n \phi}\right\rangle}{\left\langle r^{m}\right\rangle},
$$

where the minus signs is merely a convention, and here we have centered the coordinates in each event such as $\left\langle r e^{i \phi}\right\rangle=W_{1,1}=0$. The standard eccentricities are defined as the lowest term with the correct symmetry, e.g., $\epsilon_{1} \equiv \epsilon_{1,3}, \epsilon_{2} \equiv \epsilon_{2,2}, \epsilon_{3} \equiv \epsilon_{3,3}$, etc. For the lowest orders, where this definition is usually used, the numerator can be replaced with the appropriate cumulant. With these dimensionless quantities defined, we can replace $W_{n, m} \rightarrow \epsilon_{n, m}$ in Eq. (3.23), and the coefficients $\kappa_{n, m}$ are now dimensionless. This also ensures that $\epsilon_{n, m} \leq 1$ simply by its definition, which makes it a good quantity for a power series of (3.23).

Note however, in the formalism of cumulants expansion, for odd $m$, the denominator is not actually a cumulant (equal to zero). A more natural choice may be to simply take the appropriate power of $W_{0,2}$, the lowest cumulant representing the size of the system, i.e., one can define the ratio

$$
\epsilon_{n, m} \equiv-m ! \frac{W_{n, m}}{\left(W_{0,2}\right)^{\frac{m}{2}}}
$$

where

$$
\epsilon_{n}=\varepsilon_{n} e^{i n \Phi_{n}}
$$

where the phase $\Phi_{n}$ is known as participant plane, the exponent $\frac{m}{2}$ makes the eccentricity dimensionless for any $m$, and the term $m$ ! exists because each cumulant has in its front a coefficient and when we divide $W_{n, m}$ by $W_{0,2}^{\frac{m}{2}}$, it appears the term $1 / m$ ! in front, then to eliminate it, the eccentricity was defined multiplying by $m$ !.

Now, we use the same relation (3.23), choosing $m=|n|$ in order to write the lowest eccentricities (lowest cumulants), such that

$$
V_{n}=\kappa_{n} \epsilon_{n}+O\left(\epsilon^{2}\right)
$$

where the linear response coefficient $\kappa_{n}$ depend only on hydrodynamic parameters, and even it is possible to improve more this relation using superior terms of expansion [67], this work is interested only in linear response.

We have derived a relation between the initial energy density distribution, for example, and the final azimuthal anisotropy coefficients $V_{n}$, which has already been used successfully to describe hydrodynamic calculations and which has provided much insight into the 
relationship between initial and final states.

\subsection{Energy density as a generating function}

The generating function can be replaced by initial energy density written as

$$
\rho(\vec{k})=\frac{1}{2 \pi} \int d^{2} x T^{\tau \tau}(\vec{x}) e^{i \vec{k} \cdot \vec{x}}
$$

and its cumulant expansion is exactly the same.

In order to check Eq. (3.27), some simulations were performed. The initial conditions chosen do not represent a realistic collision, but we only play with the initial conditions and check the final results. This group of initial conditions was called Toy Model. Then, the energy density was chosen as a deformed Gaussian (similar made in [68]) at $\tau_{0}$ written as

$$
T^{\tau \tau}(\vec{x})=A e^{-\frac{r^{2}}{2 \sigma^{2}}\left(1+\sum_{n} a_{n} \cos n\left(\phi+\psi_{n}\right)\right)},
$$

where $A$ is the magnitude of the Gaussian, $n$ is a positive integer, $\sigma$ is the transversal size (radius), $\psi_{n}$ is a reference angle, and the term responsible for the asymmetry (magnitude of the deformations) is $a_{n}$, such that they belong to the range $0 \leq a_{n}<1$. It is important to note that a value of $a_{n}$ close to 1 imply in a Gaussian which does not converge at infinity. In this picture, whether all these terms of deformation are zero, the energy density will be symmetrically distributed in the transversal plane $\{x, y\}$ as shown in Figure 3.2.

The sign in front of $a_{n}$ in Eq. (3.30) has been chosen such that $\psi_{n}$ is the direction of the the polygon. For $n=2$, is the mirror axis of the ellipse [66], which is the standard definition of the participant plane [69].

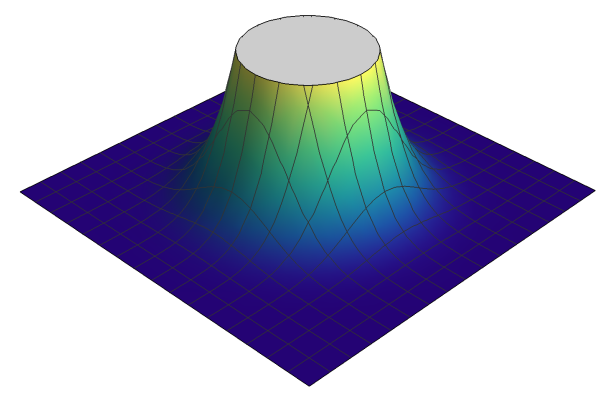

FIGURE 3.2: Profile of initial energy density at midrapidity symmetrically distributed in the transversal plane because $a_{n}=0$.

As we deal with Toy Model, this system does not represent a real collision, but its parameters $A$ and $\sigma$ (Table 3.1) have been chosen in order to create a size of a real system as [70], which corresponds roughly to a typical collision at RHIC. 
TABLE 3.1: Parameters of initial energy density symmetrically distributed.

\begin{tabular}{|c|c|c|c|}
\hline$A\left(\mathrm{fm}^{-4}\right)$ & $\sigma(\mathrm{fm})$ & $\psi_{n}$ & $a_{n}$ \\
\hline \hline 50.0 & 3.0 & 0.0 & 0.0 \\
\hline
\end{tabular}

In order to understand the shape of deformation of the Gaussian, we have two simple examples where on the other hand, in the Figure 3.3, only $a_{2}$ is different from zero and $n=2$ (left), and consequently it appears as a deformation of the Gaussian. It is important that this initial condition has as effect on the final particle distribution, meaning that all harmonic flow coefficients will be zero except $V_{2}$ because $a_{2} \neq 0$. Following the same reasoning, with only $a_{3}$ if different of zero and $n=3$ (right), and in the same way it implies in $V_{3} \neq 0$ because $a_{3} \neq 0$ and hence, all other $V_{n}$ are zero. For both cases, $V_{n}$ is real. As we shall see, $v_{n}$ is usually positive for $a_{n}>0$, which means that anisotropic flow develops along the flat side of the polygons (Figure 3.3).
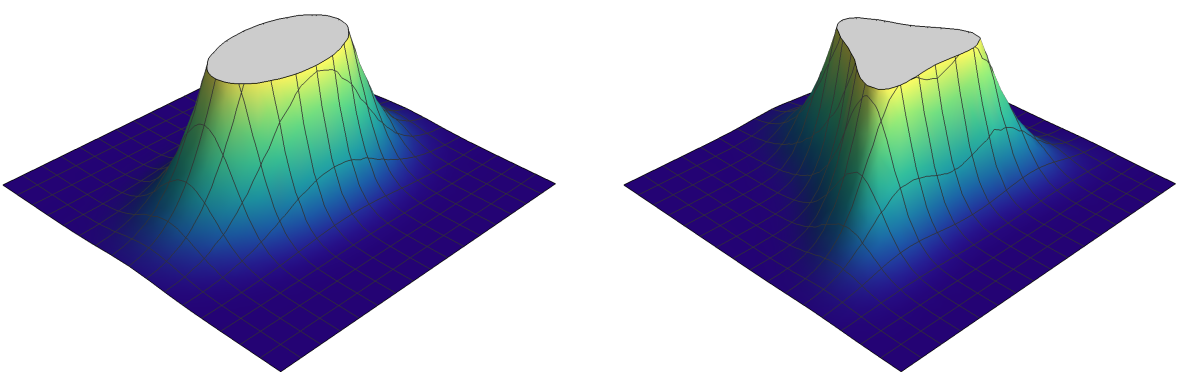

FIGURE 3.3: Profile of energy density deformed because $a_{2} \neq 0$ (left) and deformed because $a_{3} \neq 0$ (right).

Now, in order to determine whether these eccentricities of Toy Model describe the evolution of a heavy ion collision system, we perform hydrodynamic simulations. Specifically, all simulations of this model use the equation of state s95p-v1, vanishing bulk viscosity $\mathrm{s} / \mathrm{s}$, the shear viscosity $\eta / s$ used was 0.2 , and the hydrodynamics begins at $\tau_{0}=1.0 \mathrm{fm}$. In next chapter, all simulations use the same parameters in Toy Model.

The Toy Model was created to check whether the eccentricities with other $T^{\mu \nu}$ components work. Then for simplicity, we only calculate the spectrum of direct pions, without contributions of resonance decays.

We are interested to study the hydrodynamic response to initial conditions. For this simple case where we have only $T^{\tau \tau}$ contribution we can play with initial conditions, varying its parameters in order to probe the resulting response.

In general, $a_{n}$ deforms the initial energy density and generates effects in its correspondent harmonic flow. This happens because anisotropic flow $V_{n}$ is seen as a hydrodynamic response to spatial anisotropies $\epsilon_{n}$. This response function is represented by relations (3.27). In this picture, simulations were made in order to test how effective these relations are for $n=2$ and 3 . 
All eccentricities can be defined in the same way, then according to Eq. (3.27), it is possible to establish a linear relation between these predictors and the harmonic flow coefficients as

$$
v_{n} e^{i n \Psi_{n}}=\kappa_{n} \varepsilon_{n} e^{i n \Phi_{n}} .
$$

Figure 3.4 displays the relation $V_{2}$ and $\epsilon_{2}$ event-by-event, for fluid velocities $u^{x}=u^{y}=$ $u^{\eta_{s}}=0$. Using the same values of parameters as $A$ and $\sigma$, we have the following form for $\epsilon_{2}$

$$
\epsilon_{2}^{e}=-\frac{\left\langle r^{2} e^{i 2 \phi}\right\rangle_{e}-\left\langle r e^{i \phi}\right\rangle_{e}^{2}}{\left\langle r^{2}\right\rangle_{e}-\left|\left\langle r e^{i \phi}\right\rangle_{e}\right|^{2}}
$$

where

$$
\langle\ldots\rangle_{e}=\frac{\int d^{2} x \ldots T^{\tau \tau}(\vec{x})}{\int d^{2} x T^{\tau \tau}(\vec{x})} .
$$

As shown, the parameter $a_{2}$ is responsible for the asymmetry of $\epsilon_{2}$, then each event has a different value of this parameter. This form of energy density is more clear below

$$
T^{\tau \tau}(\vec{x})=A e^{-\frac{r^{2}}{2 \sigma^{2}}\left(1+a_{2} \cos 2 \phi\right)} .
$$

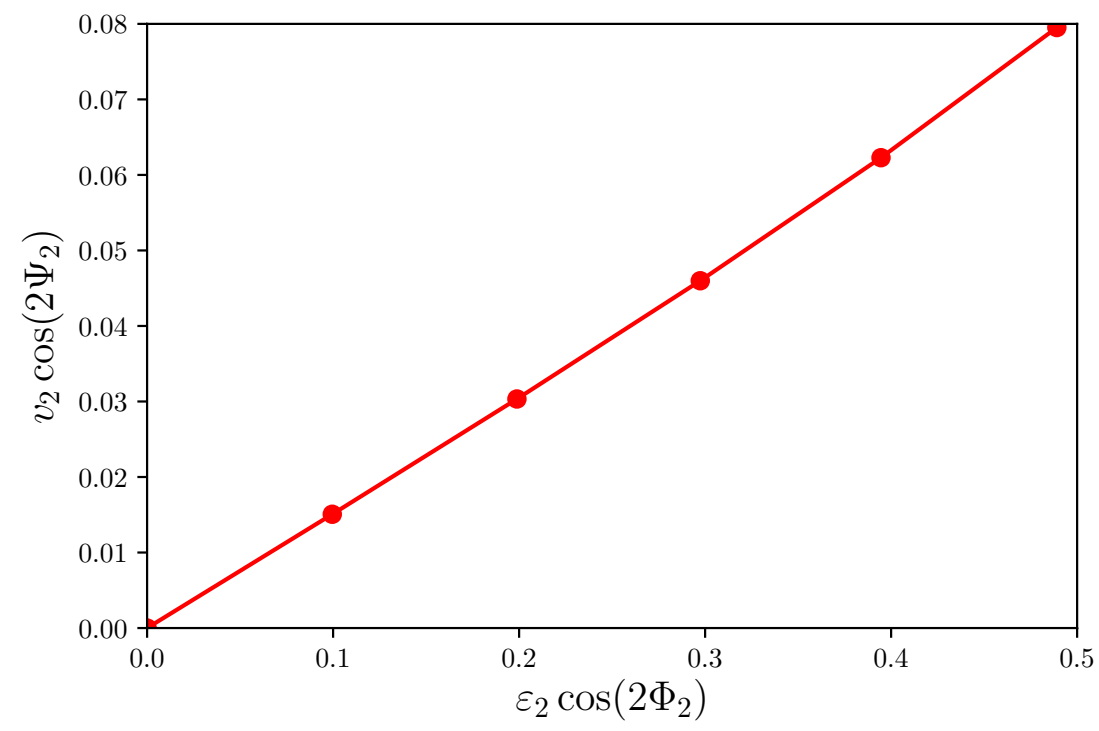

FIGURE 3.4: Relation between real projections of $V_{2}$ and $\epsilon_{2}$ event-by-event. 
TABLE 3.2: Parameters used for $V_{2} \times \epsilon_{2}$.

\begin{tabular}{|c|c|c|}
\hline$A\left(\mathrm{fm}^{-4}\right)$ & $\sigma(\mathrm{fm})$ & $a_{2}$ \\
\hline \hline 50.0 & 3.0 & $\{0.0-0.6\}$ \\
\hline
\end{tabular}

On the other hand, for $n=3$ is the same idea, where $a_{3}$ changes in the same way. It is possible to make a plot which displays the relation between the real projections of $V_{3}$ and $\epsilon_{3}$ event-by-event. Similarly, we have

$$
\epsilon_{3}^{e}=-\frac{\left\langle r^{3} e^{i 3 \phi}\right\rangle_{e}+\left\langle r e^{i \phi}\right\rangle_{e}\left(3\left\langle r^{2} e^{i 2 \phi}\right\rangle_{e}-2\left\langle r e^{i \phi}\right\rangle_{e}^{2}\right)}{\left(\left\langle r^{2}\right\rangle_{e}-\left|\left\langle r e^{i \phi}\right\rangle_{e}\right|^{2}\right)^{\frac{3}{2}}},
$$

and in shape of the Gaussian is

$$
T^{\tau \tau}(\vec{x})=A e^{-\frac{r^{2}}{2 \sigma^{2}}\left(1+a_{3} \cos 3 \phi\right)} .
$$

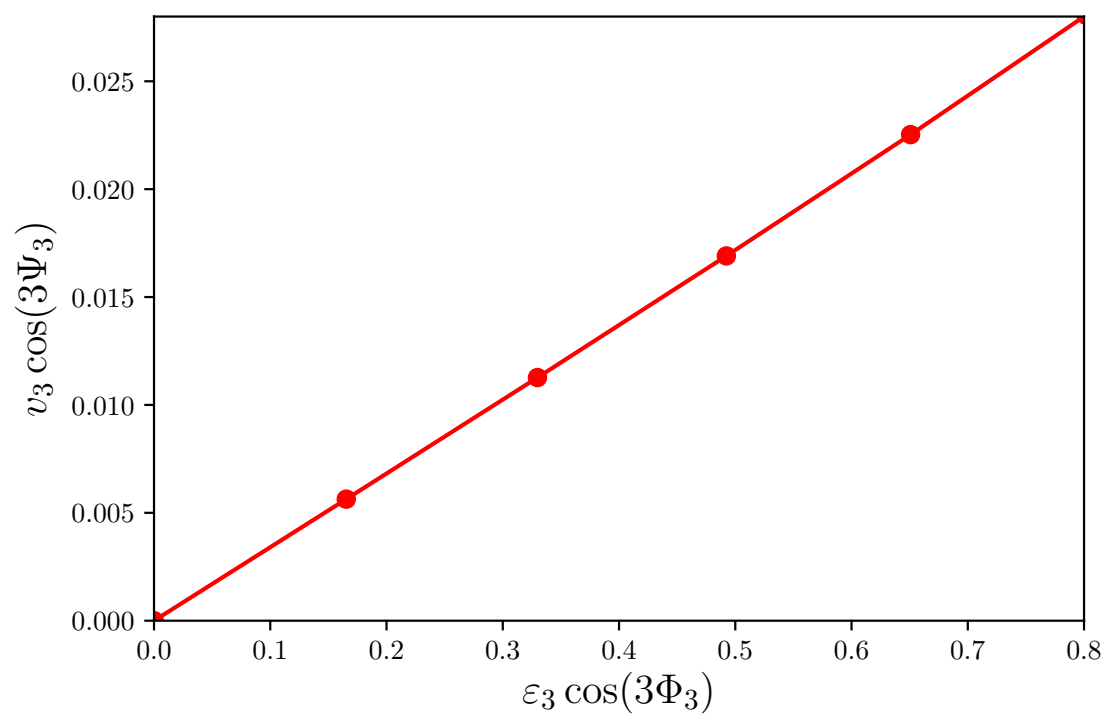

FIGURE 3.5: Relation between real projections of $V_{3}$ and $\epsilon_{3}$ event-by-event.

TABLE 3.3: Parameters used for $V_{3} \times \epsilon_{3}$.

\begin{tabular}{|c|c|c|}
\hline$A\left(\mathrm{fm}^{-4}\right)$ & $\sigma(\mathrm{fm})$ & $a_{3}$ \\
\hline \hline 50.0 & 3.0 & $\{0.0-0.6\}$ \\
\hline
\end{tabular}

We used the same parameters for $A$ and $\sigma$ of the table 3.1.

It is possible to note the linear relation between $\epsilon_{n}$ and $V_{n}$. This shows that in Toy Model, the hydrodynamic response to initial energy density is consistent. 


\section{Chapter 4}

\section{Including effects from other $T^{\mu \nu}$ components}

We are interested in constructing a framework which incorporates the effects of initial momentum density and initial stress tensor. Using the same considerations as for the case of energy density only, these other components of initial $T^{\mu \nu}$ were included through a nontrivial ansatz that added one term for each new contribution, following the cumulant expansion exactly as made before

$$
\rho(\vec{x})=T^{\tau \tau}+\alpha \partial_{i} T^{\tau i}-\beta \partial_{i} \partial_{j} T^{i j} .
$$

Because we do not know the relative importance of each new contribution, each one has a constant response coefficient $\alpha(\mathrm{fm})$ and $\beta\left(\mathrm{fm}^{2}\right)$ with the appropriate dimension. Assuming Eq. (4.1), we can begin to construct the framework, but it is advisable to add one contribution at a time in order to understand better its effect.

\subsection{Physical motivation}

Let us return to the case where only energy density contributes, such that the evolution beginning at some time $\tau_{0}$ and depends on the distribution of energy density only. We know that these estimators $\epsilon_{n}^{e}\left(\tau_{0}\right)$ give an excellent description of the final results. Now, imagine that we do not know the value of $\tau_{0}$, we instead generate estimators $\epsilon_{n}^{e}(\tau)$ from the state of the system a short time before or after $\tau_{0}$ such as $\tau=\tau_{0}+\delta \tau$, and in this way we have a new expression for energy density

$$
T^{\tau \tau}(\tau)=T^{\tau \tau}\left(\tau_{0}\right)+\left.\delta \tau \partial_{\tau} T^{\tau \tau}\right|_{\tau_{0}}+\left.\frac{1}{2} \delta \tau^{2} \partial_{\tau}^{2} T^{\tau \tau}\right|_{\tau_{0}}+O\left(\delta \tau^{3}\right)
$$

The final state of the system is the same, and so our estimator should be approximately unchanged.

Conservation of $T^{\mu \nu}$ show us that, to first order, any change is energy density is compesated by momentum density. And we can continue to second order, which adds the stress tensor and we obtain

$$
\begin{aligned}
& \partial_{\tau} T^{\tau \tau}=-\partial_{i} T^{\tau i}, \\
& \partial_{\tau}^{2} T^{\tau \tau}=\partial_{i} \partial_{j} T^{i j} .
\end{aligned}
$$


Thus, our original scalar $\rho(\vec{x})$ can be approximated by these terms

$$
\rho(\vec{x})=T^{\tau \tau}\left(\tau_{0}\right) \simeq T^{\tau \tau}(\tau)+\delta \tau \partial_{i} T^{\tau i}\left(\tau_{0}\right)+\frac{1}{2} \delta \tau^{2} \partial_{i} \partial_{j} T^{i j}\left(\tau_{0}\right) .
$$

So while we cannot make a rigorous derivation of, e.g., the values of $\alpha$ and $\beta$, which clearly depend on the system, it is natural to construct a field with a some of the three quantities in the form of Eq. (4.1) $\left(T^{\tau \tau}, T^{\tau i}\right.$, and $\left.T^{i j}\right)$ to make an estimator for the final flow.

While not all transverse degrees of freedom of $T^{\mu \nu}$ appears, these three quantities can be expected to be the most important.

\subsection{Effects from initial momentum density}

The vector field $T^{\tau i}$, with $i \in\{x, y\}$ transforms nontrivially with respect to azimuthal rotations. This must be taken into account to obtain cumulants with well-defined rotational properties. Using the general notation for the momentum density $U(\vec{x})$, we start by defining a complex number representing the (2-D) momentum density vector as

$$
U(\vec{x})=T^{\tau x}+i T^{\tau y}
$$

and the cumulant expansion needs to consider the rotational property of $U(\vec{x})$ : if the system rotates such as $\phi \rightarrow \phi+\delta$, this function transforms as $U(\vec{x}) \rightarrow U(\vec{x}) e^{i \delta}$. In the generating function, this quantity appears in the divergent $\partial_{i} T^{\tau i}$ which is a scalar.

In this section, we neglected the importance of the initial stress tensor to isolate the effects caused by anisotropy from the initial momentum density. Thus, in this case, the generating function can be written as

$$
\rho(\vec{x})=T^{\tau \tau}+\alpha \partial_{i} T^{\tau i},
$$

Taking the Fourier transform and this equation changes to

$$
\rho(\vec{k})=\frac{1}{2 \pi} \int d^{2} x\left(T^{\tau \tau}-i \alpha k_{i} T^{\tau i}\right) e^{i \vec{k} \cdot \vec{x}} .
$$

In order to select only the large-scale structure and assuming that this function is well behaved, we expand it in a Maclaurin series around $|\vec{k}|=0$ and to identify quantities with correct rotational properties, we expand the Maclaurin coefficients in Fourier series with respect to $\phi_{k}$ and obtain

$$
\rho(\vec{k})=\sum_{m=0}^{\infty} \sum_{n=-\infty}^{\infty} \rho_{n, m}(\alpha) k^{m} e^{-i n \phi_{k}}
$$


And now we have a general form for its moments written as

$$
\begin{aligned}
\rho_{n, m}(\alpha)= & \frac{i^{m}}{2^{m}\left(\frac{m+n}{2}\right) !\left(\frac{m-n}{2}\right) !}\left[\int d^{2} x r^{m} e^{i n \phi} T^{\tau \tau}(\vec{x})\right. \\
& -\alpha\left(\frac{m+n}{2}\right) \int d^{2} x r^{m-1} e^{i(n-1) \phi} U(\vec{x}) \\
& \left.-\alpha\left(\frac{m-n}{2}\right) \int d^{2} x r^{m-1} e^{i(n+1) \phi} U^{*}(\vec{x})\right],
\end{aligned}
$$

More details are provided in appendix B.

In the same way as for energy energy density, moments $\rho_{n, m}$ for $m \geq|n|$ and when the difference $(m-n)$ is an even number exist. In addition, there is the same problem with the moments because they are not translation-invariant and depend of the center of coordinates, and the same solution is used here which consists of a switch of moments by cumulants of the generating function.

$$
\rho(\vec{k})=e^{W(\vec{k})}
$$

Expanding $W(\vec{k})$ in Maclaurin and Fourier series,

$$
W(\vec{k})=\sum_{m=0}^{\infty} \sum_{n=-\infty}^{\infty} W_{n, m}(\alpha) k^{m} e^{-i n \phi_{k}} .
$$

Therefore, the first few cumulants are

$$
W_{0,0}(\alpha)=\ln [\rho(\vec{k}=\overrightarrow{0})],
$$

as we can note, the cumulant $W_{0,0}$ does not change, but all others have

$$
\begin{gathered}
W_{1,1}(\alpha)=\frac{i}{2}\left[\left\langle r e^{i \phi}\right\rangle_{e}-\langle\alpha\rangle_{u}\right] \\
W_{0,2}(\alpha)=\frac{1}{2} \frac{i^{2}}{2 !}\left[\left\langle r^{2}\right\rangle_{e}-\alpha\left\langle r e^{-i \phi}\right\rangle_{u}-\alpha\left\langle r e^{i \phi}\right\rangle_{u^{*}}-\left|\left\langle r e^{i \phi}\right\rangle_{e}-\langle\alpha\rangle_{u}\right|^{2}\right] \\
W_{2,2}(\alpha)=\frac{1}{4} \frac{i^{2}}{2 !}\left[\left\langle r^{2} e^{i 2 \phi}\right\rangle_{e}-2 \alpha\left\langle r e^{i \phi}\right\rangle_{u}-\left(\left\langle r e^{i \phi}\right\rangle_{e}-\langle\alpha\rangle_{u}\right)^{2}\right] \\
W_{3,3}(\alpha)=\frac{1}{8} \frac{i^{3}}{3 !}\left[\left\langle r^{3} e^{i 3 \phi}\right\rangle_{e}-3 \alpha\left\langle r^{2} e^{i 2 \phi}\right\rangle_{u}-\left(\left\langle r e^{i \phi}\right\rangle_{e}-\langle\alpha\rangle_{u}\right)\right. \\
\left.\cdot\left(3\left(\left\langle r^{2} e^{i 2 \phi}\right\rangle_{e}-2 \alpha\left\langle r e^{i \phi}\right\rangle_{u}\right)-2\left(\left\langle r e^{i \phi}\right\rangle_{e}-\langle\alpha\rangle_{u}\right)^{2}\right)\right]
\end{gathered}
$$

where

$$
\langle\ldots\rangle_{u}=\frac{\int d^{2} x \ldots U(\vec{x})}{\int d^{2} x T^{\tau \tau}(\vec{x})}
$$




$$
\langle\ldots\rangle_{u^{*}}=\frac{\int d^{2} x \ldots U^{*}(\vec{x})}{\int d^{2} x T^{\tau \tau}(\vec{x})} .
$$

Where like shown previously, momentum density is represented by the complex number $U(\vec{x})$.

The idea is the same of Chapter 3 which is to construct estimators that are able to predict the final state. However this case is different because it contains a new contribution and the goal is to check if this framework works.

Firstly, we can define the eccentricities in the same way

$$
\epsilon_{n}(\alpha)=-n ! \frac{W_{n, n}(\alpha)}{\left(W_{0,2}^{\epsilon}\right)^{\frac{n}{2}}}
$$

A important detail is that the denominator does not have the momentum density contribution because these new terms are negative, and as consequence events which $W_{0,2}$ is really close to zero would have eccentricities going to infinity but the harmonic flow coefficients do not. Therefore, the complete denominator is inappropriate for estimators $\epsilon_{n}$, making $W_{0,2}^{e}$ to be the best option.

In this work, $\epsilon_{2}$ and $\epsilon_{3}$ are the most important eccentricities to verify in simulations the best value of the constant $\alpha$, thus we have

$$
\epsilon_{2}(\alpha)=-\frac{\left\langle r^{2} e^{i 2 \phi}\right\rangle_{e}-2 \alpha\left\langle r e^{i \phi}\right\rangle_{u}-\left(\left\langle r e^{i \phi}\right\rangle_{e}-\langle\alpha\rangle_{u}\right)^{2}}{\left\langle r^{2}\right\rangle_{e}-\left|\left\langle r e^{i \phi}\right\rangle_{e}\right|^{2}}
$$

in the same way, $\epsilon_{3}$ can be written as

$$
\begin{aligned}
\epsilon_{3}(\alpha) & =-\frac{\left\langle r^{3} e^{i 3 \phi}\right\rangle_{e}-3 \alpha\left\langle r^{2} e^{i 2 \phi}\right\rangle_{u}-\left(\left\langle r e^{i \phi}\right\rangle_{e}-\langle\alpha\rangle_{u}\right)\left[3\left\langle r^{2} e^{i 2 \phi}\right\rangle_{e}-6 \alpha\left\langle r e^{i \phi}\right\rangle_{u}\right.}{\left(\left\langle r^{2}\right\rangle_{e}-\left|\left\langle r e^{i \phi}\right\rangle_{e}\right|^{2}\right)^{\frac{3}{2}}} \\
& \frac{\left.-2\left(\left\langle r e^{i \phi}\right\rangle_{e}-\langle\alpha\rangle_{u}\right)^{2}\right]}{\left(\left\langle r^{2}\right\rangle_{e}-\left|\left\langle r e^{i \phi}\right\rangle_{e}\right|^{2}\right)^{\frac{3}{2}}} .
\end{aligned}
$$

In order to check if the framework works in event-by-event simulations, it is necessary to play with initial conditions (Toy Model), where the energy density is the same used in Chapter 3

$$
T^{\tau \tau}(\vec{x})=A e^{-\frac{r^{2}}{2 \sigma^{2}}\left(1+\sum_{n} a_{n} \cos n \phi\right)},
$$

and as the momentum density is a complex function, then it can be written as a function of magnitude and phase as

$$
U(\vec{x})=|U| e^{i \phi_{u}},
$$

where, the magnitude is a function which is proportional to a Gaussian as

$$
|U|=r B e^{-\frac{r^{2}}{2 \rho^{2}}\left(1-\sum_{n} b_{n} \cos n \phi\right)},
$$


and the phase is

$$
\phi_{u}=\phi-\sum_{n} c_{n} \sin n \phi
$$

where $B$ is the magnitude of momentum density, $n$ is a positive integer, $\rho$ is the transverse size (radius of momentum density), the term responsible for the deformation is $b_{n}$ in magnitude of $U(\vec{x})$, such that they belong to the range $0 \leq b_{n}<1$, and $c_{n}$ is the term responsible for the deformation in direction. It is important to note that values of $b_{n}$ close to 1 imply in a function which does not converge at infinity. In this picture, whether $b_{n}$ and $c_{n}$ are zero, the momentum density will be symmetrically distributed in the transversal plane $\{x, y\}$ as shown at Figure 4.1.

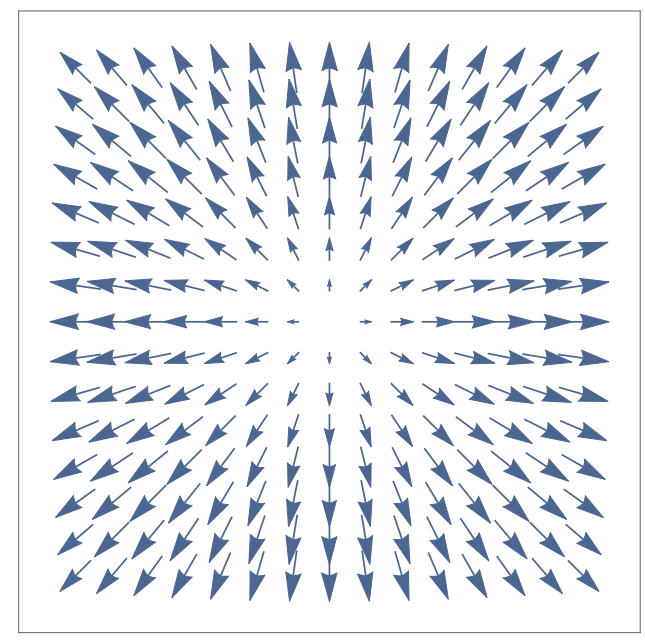

FIGURE 4.1: Profile of momentum density symmetrically distributed because $b_{n}$ and $c_{n}$ are zero.

TABLE 4.1: Parameters used for momentum density.

\begin{tabular}{|c|c|c|c|}
\hline$B\left(\mathrm{fm}^{-5}\right)$ & $\rho(\mathrm{fm})$ & $b_{n}$ & $c_{n}$ \\
\hline \hline 10.0 & 1.0 & 0.0 & 0.0 \\
\hline
\end{tabular}

Basically, as we are using the same parameters of Chapter 4 for energy density that corresponds to a typical collision at RHIC. Using this idea, the parameters used for momentum density $(B, \rho)$ naturally have a small size compared to energy density $(A, \sigma)$ because it is more common in realistic events, and this same idea will be used for parameters of stress tensor in next section.

In Figure 4.1, $b_{n}$ and $c_{n}$ are zero, which it implies that the correspondent harmonic flow coefficient $V_{n}$ is also zero. In this picture, Figure 4.2 has only $b_{2} \neq 0$ (left), and it implies in $V_{2} \neq 0$. And analogously, the right side has only $b_{3} \neq 0$ and hence it produces $V_{3} \neq 0$.

Keeping the same idea, the term $c_{n}$ of phase are also able to generate anisotropic flow. Figure 4.3 has only $c_{2} \neq 0$, and it implies in $V_{2}=0$ (left), and the right side has only $c_{3} \neq 0$ what makes $V_{3} \neq 0$. The same parameters from Table 4.1 were used for both figures. 


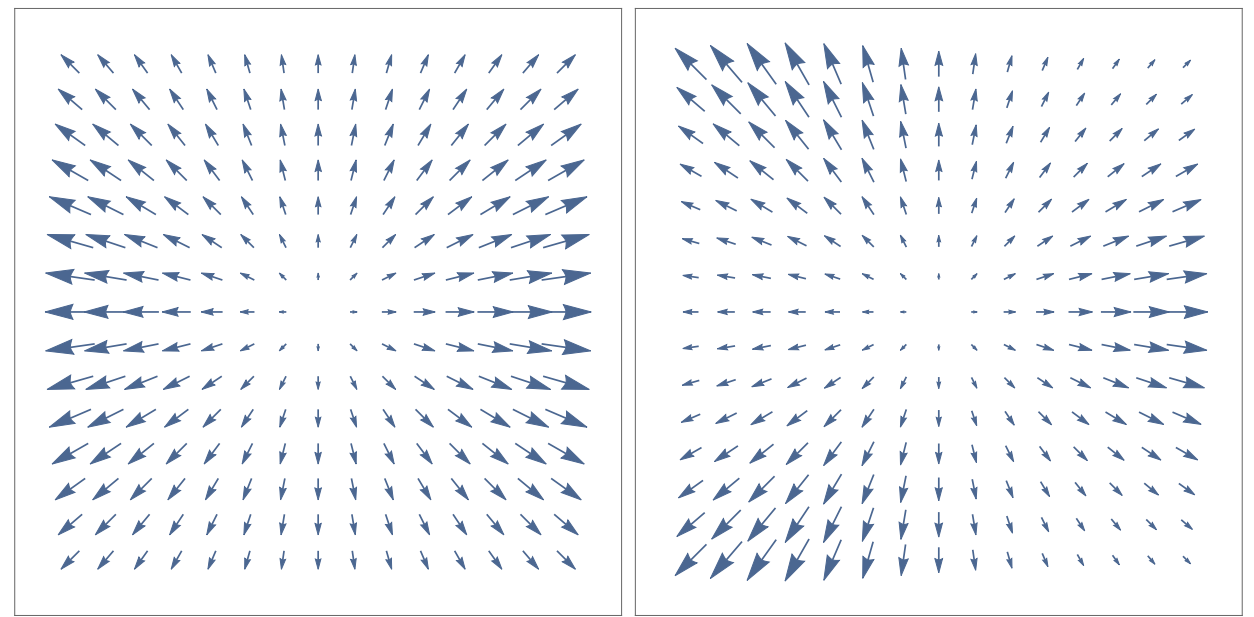

FIGURE 4.2: Profile of momentum density asymmetrically distributed because $b_{2} \neq 0$ (left) and because $b_{3} \neq 0$ (right).

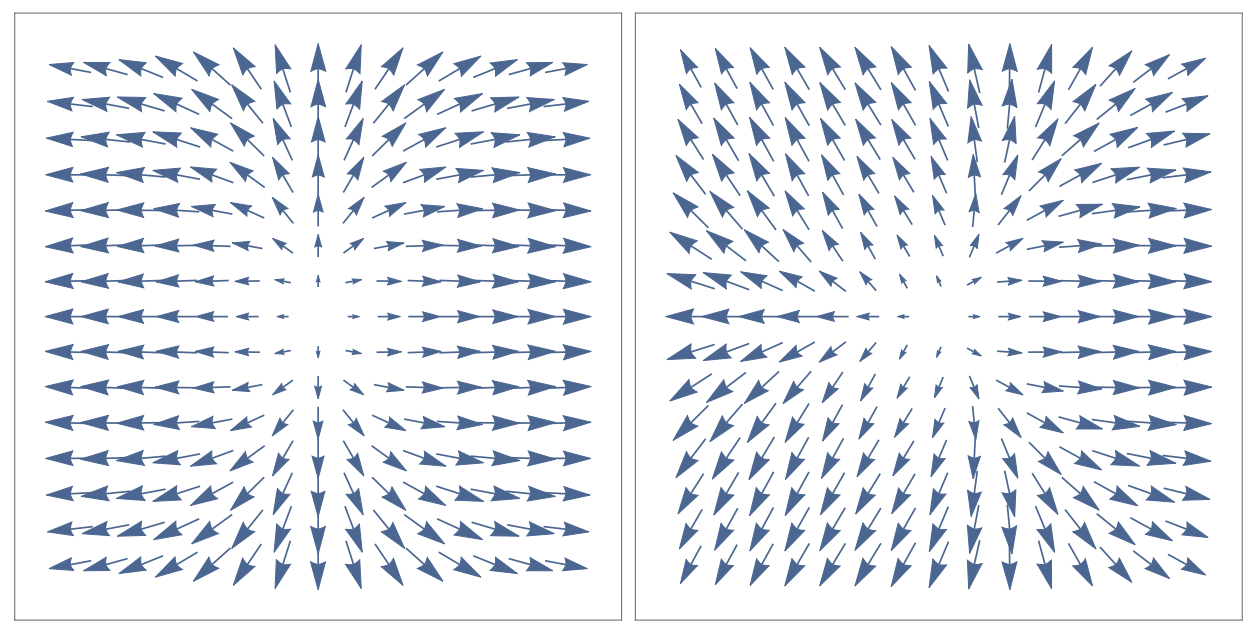

FIGURE 4.3: Profile of momentum density asymmetrically distributed because $c_{2} \neq 0$ (left) and because $c_{3} \neq 0$.

It was used the equation of state $\mathrm{s} 95 \mathrm{p}-\mathrm{v} 1$, vanishing bulk viscosity $\varsigma / s$, the shear viscosity $\eta / s$ used was 0.2 , and the hydrodynamics begins at $\tau_{0}=1.0 \mathrm{fm}$

In order to check the relation between $V_{n}$ and $\epsilon_{n}(\alpha)$, simulations were made, changing all relevant parameters of initial conditions. Figure 4.4 plots events made for $n=2$. In particular, we have centered the coordinate system such that $W_{1,1}(\alpha)=0$

$$
\epsilon_{2}(\alpha)=-\frac{\left\langle r^{2} e^{i 2 \phi}\right\rangle_{e}-2 \alpha\left\langle r e^{i \phi}\right\rangle_{u}}{\left\langle r^{2}\right\rangle_{e}-\left|\left\langle r e^{i \phi}\right\rangle_{e}\right|^{2}},
$$


where the parameters are

$$
\begin{aligned}
T^{\tau \tau} & =A e^{-\frac{r^{2}}{2 \sigma^{2}}\left(1+a_{2} \cos 2 \phi\right)} \\
|U| & =r B e^{-\frac{r^{2}}{2 \rho^{2}}\left(1-b_{2} \cos 2 \phi\right)} \\
\phi_{u} & =\phi-c_{2} \sin 2 \phi .
\end{aligned}
$$

This eccentricity is able to capture effects from energy and momentum density, but if $\alpha=0$ we have correspondence with the usual case which we had only energy density contribution.
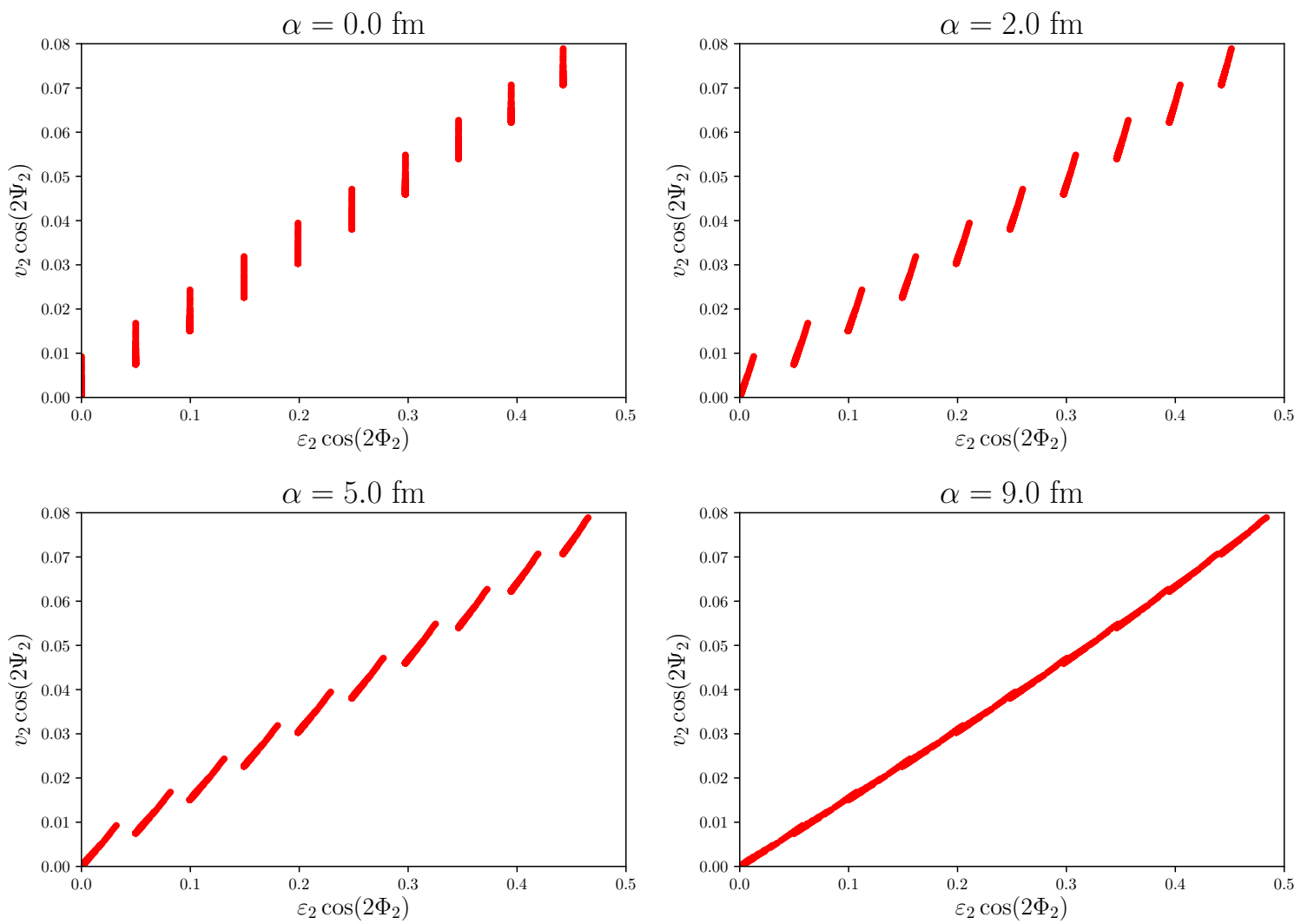

FiguRE 4.4: Plots of $V_{2} \times \epsilon_{2}(\alpha=0)$ (upper left), of $V_{2} \times \epsilon_{2}(\alpha=2.0) \mathrm{fm}$ (upper right), of $V_{2} \times \epsilon_{2}(\alpha=5.0) \mathrm{fm}$ (lower left), and of $V_{2} \times \epsilon_{2}(\alpha=9.0)$ fm (lower right).

TABLE 4.2: Parameters used for $V_{2} \times \epsilon_{2}(\alpha) \mathrm{fm}$.

\begin{tabular}{|c|c|c|c|}
\hline$B\left(\mathrm{fm}^{-5}\right)$ & $a_{2}$ & $b_{2}$ & $c_{2}$ \\
\hline \hline$\{0.0-10.0\}$ & $\{0.0-0.6\}$ & $\{0.0-0.6\}$ & $\{0.0-0.4\}$ \\
\hline
\end{tabular}

Each point in these scatters (Figure 4.4) represents a different event where we changed values for all parameters. It is possible to realize that for the first case which has $\alpha=$ 
$0, V_{2}$ has points in vertical, it happens because the elliptic flow coefficient is sensitive to asymmetries from initial momentum density, but in this case $(\alpha=0)$, the eccentricity does not capture these effects. On the other hand, when $\alpha=2.0$ and $5.0 \mathrm{fm}, \epsilon_{2}$ captures these effects even there is not a linear relationship between $V_{2}$ and $\epsilon_{2}$, but it is possible to figure out the best value of $\alpha$ where in Figure 4.4 it is possible to obtain a linear relationship.

Now, for $n=3$, the behavior is similar. Figure 4.5 plots the relation $V_{3} \times \epsilon_{3}$ event-byevent changing all relevant parameters of initial conditions, we have

$$
\epsilon_{3}(\alpha)=-\frac{\left\langle r^{3} e^{i 3 \phi}\right\rangle_{e}-3 \alpha\left\langle r^{2} e^{i 2 \phi}\right\rangle_{u}}{\left(\left\langle r^{2}\right\rangle_{e}-\left|\left\langle r e^{i \phi}\right\rangle_{e}\right|^{2}\right)^{\frac{3}{2}}} .
$$

And analogously as made with the parameters of $\epsilon_{2}$, for $\epsilon_{3}$, we have

In order to confirm that $\alpha$ is a constant independent of harmonic, it was used the best value of $\epsilon_{2}$.

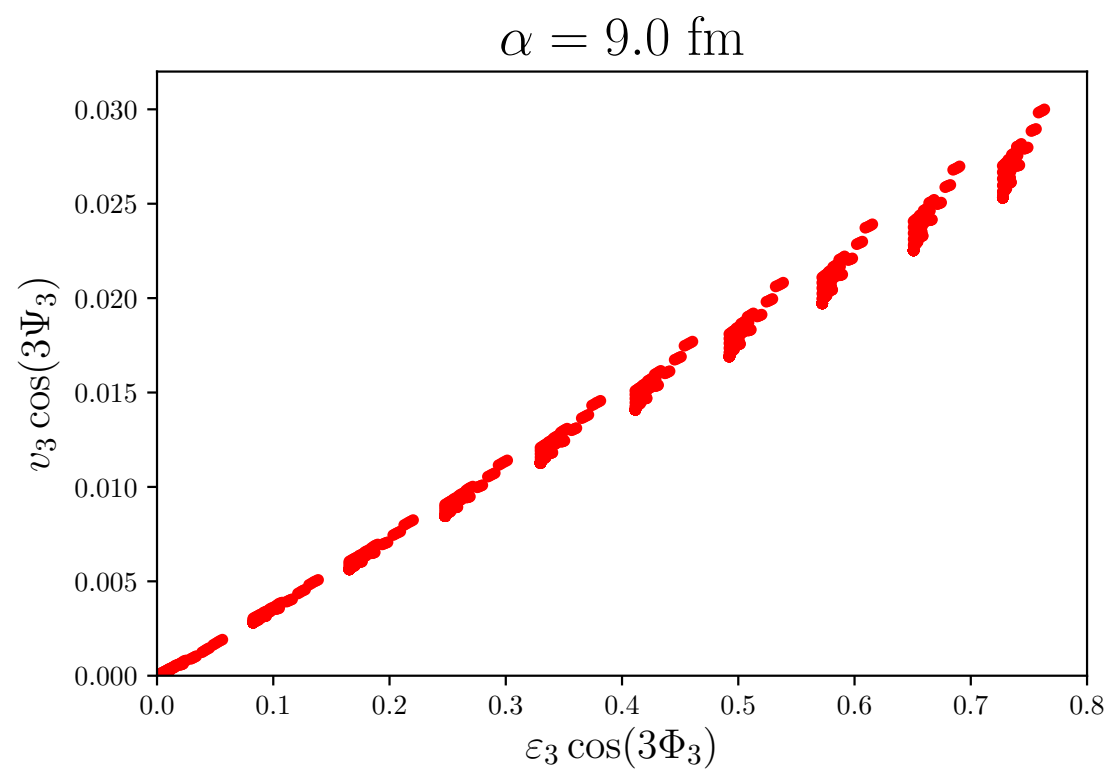

FIGURE 4.5: Relation between $V_{3}$ and $\epsilon_{3}(\alpha)$ event-by-event.

TABLE 4.3: Parameters used for $V_{3} \times \epsilon_{3}(\alpha)$.

\begin{tabular}{|c|c|c|c|}
\hline$B\left(f m^{-5}\right)$ & $a_{3}$ & $b_{3}$ & $c_{3}$ \\
\hline \hline$\{0.0-10.0\}$ & $\{0.0-0.6\}$ & $\{0.0-0.6\}$ & $\{0.0-0.4\}$ \\
\hline
\end{tabular}

These simulations perform the same initial parameters of MUSIC used in previous chapter. After checking these results, it was clear that relation between $V_{n} \times \epsilon_{n}(\alpha)$ is linear for $\alpha=9.0 \mathrm{fm}$ which confirms that the ansatz (4.7) works. 


\subsection{Effects from initial Stress tensor}

The quantity $T^{i j}$ for $i, j \in\{1,2\}$ is a rank 2 tensor which needs to have its rotation property respected in its eccentricities. At the generating function, this quantity appears through two consecutive contractions $\partial_{i} \partial_{j} T^{i j}$ which is scalar.

In this section, the contribution of momentum density was neglected to analyze better only the inclusion of stress tensor. As the same way as made previously, the generating function was written as

$$
\rho(\vec{x})=T^{\tau \tau}-\beta \partial_{i} \partial_{j} T^{i j},
$$

and in order to identify the correct scales of structure, it was taken Fourier transform, thus Eq. (4.30) becomes

$$
\rho(\vec{k})=\frac{1}{2 \pi} \int d^{2} x\left(T^{\tau \tau}+\beta k_{i} k_{j} T^{i j}\right) e^{i \vec{k} \cdot \vec{x}},
$$

and to select large-scales of system, it was taken Maclaurin series and to identify quantities with the correct rotational properties it was taken Fourier series

$$
\rho(\vec{k})=\sum_{m=0}^{\infty} \sum_{n=-\infty}^{\infty} \rho_{n, m}(\beta) k^{m} e^{-i n \phi_{k}} .
$$

It is convenient to separate the stress tensor in to parts: traceless and trace part as

$$
T^{i j}=\widetilde{T}^{i j}+\frac{T}{2} \delta^{i j}
$$

where $T=T^{x x}+T^{y y}$.

We have a general form for general moment written as

$$
\begin{aligned}
\rho_{n, m}(\beta)= & \frac{i^{m}}{2^{m}\left(\frac{m+n}{2}\right) !\left(\frac{m-n}{2}\right) !}\left[\int d^{2} x r^{m} e^{i n \phi} T^{\tau \tau}(\vec{x})\right. \\
& -\beta(m+n)\left(\frac{m+n}{2}-1\right) \int d^{2} x r^{m-2} e^{i(n-2) \phi} C(\vec{x}) \\
& -\beta(m-n)\left(\frac{m-n}{2}-1\right) \int d^{2} x r^{m-2} e^{i(n+2) \phi} C^{*}(\vec{x}) \\
& \left.-\beta(m+n)(m-n) \int d^{2} x r^{m-2} e^{i n \phi} \frac{T}{2}(\vec{x})\right],
\end{aligned}
$$

where the complex number $C(\vec{x})$ is from contractions of $\widetilde{T}^{i j}$ and is defined as

$$
C(\vec{x}) \equiv \frac{1}{2}\left(\widetilde{T}^{x x}-\widetilde{T}^{y y}\right)+i \widetilde{T}^{x y} .
$$

It is possible to see all detail of this equations in appendix B. 
Cumulants for $n=0$ and $n=1$ do not have contributions of stress tensor. Then, since for $n=2$, we have

$$
\begin{gathered}
\rho_{0,2}(\beta)=\frac{1}{2} \frac{i^{2}}{2 !}\left[\left\langle r^{2}\right\rangle_{e}-4\langle\beta\rangle_{t}-\left|\left\langle r e^{i \phi}\right\rangle_{\epsilon}\right|^{2}\right], \\
\rho_{2,2}(\beta)=\frac{1}{4} \frac{i^{2}}{2 !}\left[\left\langle r^{2} e^{i 2 \phi}\right\rangle_{e}-4\langle\beta\rangle_{c}-\left\langle r e^{i \phi}\right\rangle_{e}\right], \\
W_{3,3}(\beta)=\frac{1}{8} \frac{i^{3}}{3 !}\left[\left\langle r^{3} e^{i 3 \phi}\right\rangle_{e}-12 \beta\left\langle r e^{i \phi}\right\rangle_{c}\right. \\
\left.-\left\langle r e^{i \phi}\right\rangle_{e}\left(3\left\langle r^{2} e^{i 2 \phi}\right\rangle_{e}-2\left\langle r e^{i \phi}\right\rangle_{e}^{2}\right)\right],
\end{gathered}
$$

where

$$
\begin{aligned}
\langle\ldots\rangle_{c} & =\frac{\int d^{2} x \ldots C(\vec{x})}{\int d^{2} x T^{\tau \tau}(\vec{x})}, \\
\langle\ldots\rangle_{t} & =\frac{\int d^{2} x \ldots \frac{T}{2}(\vec{x})}{\int d^{2} x T^{\tau \tau}(\vec{x})} .
\end{aligned}
$$

Now, we can construct eccentricities with this new contribution as

$$
\epsilon_{n}(\beta)=-n ! \frac{W_{n, n}(\beta)}{\left(W_{0,2}^{e}\right)^{\frac{n}{2}}} .
$$

Analogously as done before, the denominator does not have the stress tensor contribution because it is negative and hence events which $W_{0,2}$ is really close to zero, making the eccentricity go to infinity. In this picture, the complete denominator is inappropriate for the estimators and therefore $W_{0,2}^{e}$ is also the best option.

Basically, the contribution of trace part has effects only over $\epsilon_{1}$ because all cumulants $W_{n, n}^{c}$ do not have such contribution.

For $n=2$ and 3 , these estimators are

$$
\epsilon_{2}(\beta)=-\frac{\left\langle r^{2} e^{i 2 \phi}\right\rangle_{e}-4\langle\beta\rangle_{c}-\left\langle r e^{i \phi}\right\rangle_{e}^{2}}{\left\langle r^{2}\right\rangle_{e}-\left|\left\langle r e^{i \phi}\right\rangle_{e}\right|^{2}}
$$

and

$$
\epsilon_{3}(\beta)=-\frac{\left\langle r^{3} e^{13 \phi}\right\rangle_{e}-12 \beta\left\langle r e^{i \phi}\right\rangle_{c}-\left\langle r e^{i \phi}\right\rangle_{e}\left[3\left\langle r^{2} e^{2 i \phi}\right\rangle_{e}-12\langle\beta\rangle_{c}-2\left\langle r e^{i \phi}\right\rangle_{e}^{2}\right]}{\left(\left\langle r^{2}\right\rangle_{e}-\left|\left\langle r e^{i \phi}\right\rangle_{e}\right|^{2}\right)^{\frac{3}{2}}} .
$$

In order to make some simulations with initial conditions and check if the framework works, initial conditions were chosen. The energy density has the same form where $a_{n}$ is responsible by asymmetry. On the other hand, for initial conditions of stress tensor was introduced viscosity tensor components: $\Pi^{x x}$, $\Pi^{y y}$, and $\Pi^{x y}$. The traceless part can be represented by a complex function $C(\vec{x})$ that can be written in terms of a magnitude and a phase such that

$$
C(\vec{x})=|C| e^{i 2 \phi_{c}},
$$


where each component of the tensor also can be written as the same way

$$
\begin{aligned}
& T^{x x}=2|C| \cos ^{2} \phi_{c}, \\
& T^{y y}=2|C| \sin ^{2} \phi_{c}, \\
& T^{x y}=2|C| \sin \phi_{c} \cos \phi .
\end{aligned}
$$

This magnitude is proportional to Gaussian as

$$
|C|=r^{2} P e^{-\frac{r^{2}}{2 \varrho^{2}}\left(1-\sum_{n} p_{n} \cos n \phi\right)},
$$

and the same idea was applied for its phase defined as

$$
\phi_{c}=\phi-\sum_{n} q_{n} \sin n \phi
$$

where $P$ is the magnitude $|C|, n$ is a positive integer, $\varrho$ is the transverse size, the term responsible for the deformation of magnitude is $p_{n}$, and $q_{n}$ is responsible for asymmetries in phase. Values of $p_{n}$ close to 1 imply in a function which does not converge at infinity. If all terms of asymmetry are zero, the tensor will be symmetrically distributed in the transversal plane $\{x, y\}$. However, it is not possible to show geometric effects as made before because it deals with a tensor of rank 2, but fortunately, it is possible to show these effects only in magnitude, as done in Figure 4.6 where $p_{n}=0$ and hence, this magnitude is symmetrically distributed. Basically, as we are using the same parameters of Chapter 4 for energy density,

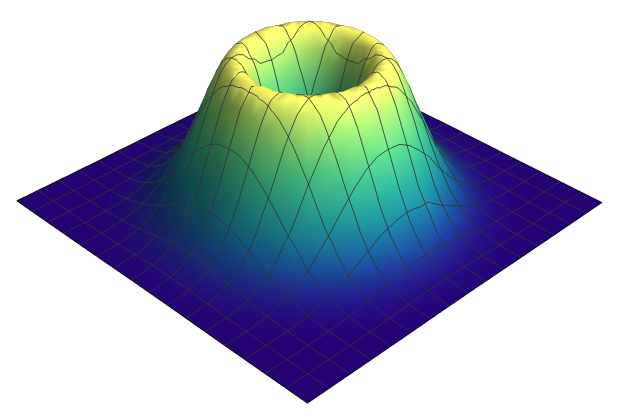

FIGURE 4.6: Profile of magnitude of a symmetrically distributed stress tensor because $p_{n}=0$.

TABLE 4.4: Parameters used for magnitude of stress tensor.

\begin{tabular}{|c|c|c|}
\hline$P\left(\mathrm{fm}^{-6}\right)$ & $\varrho(\mathrm{fm})$ & $p_{n}$ \\
\hline \hline 10.0 & 1.0 & 0.0 \\
\hline
\end{tabular}

so the parameter used for stress tensor in Table $4.4(P, \varrho)$ have a small size compared to 
energy density $(A, \sigma)$ because it is more common in typical events the size of energy density been bigger. And with this profile, all harmonic flows coefficients $V_{n}$ also zero. With the same idea, using the same parameters from Table 4.4, Figure 4.7 (left) has $p_{2} \neq 0$ and it implies that $V_{2} \neq 0$, and on the right side has $p_{3} \neq 0$ and $V_{3} \neq 0$ as consequence.
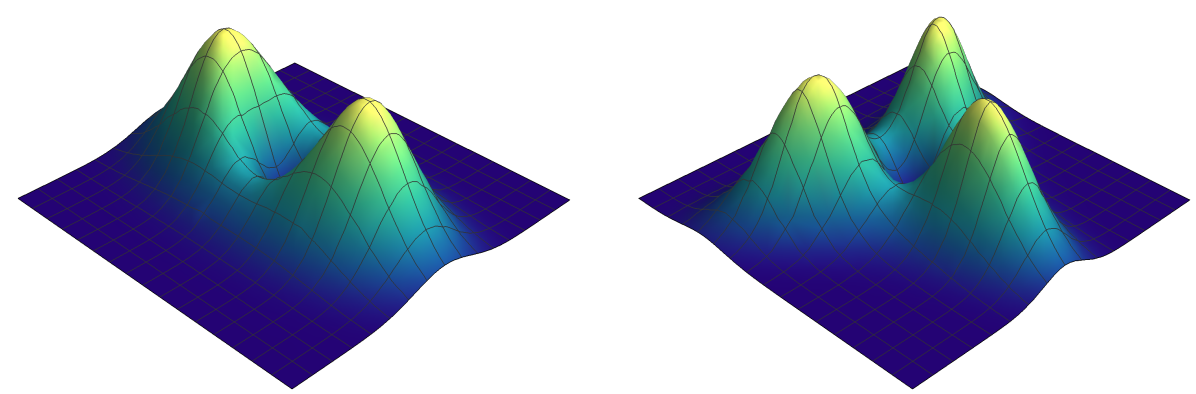

FIGURE 4.7: Profile of a asymmetrically distributed stress tensor because $p_{2} \neq 0$ (left) and because $p_{3} \neq 0$ (right).

In order to check the relation between $V_{n}$ and $\epsilon_{n}(\beta)$ simulations were done, changing all relevant parameters of initial conditions. Figure 4.8 plots events made for $n=2$ and in particular centered coordinate system such $W_{1,1}=0$, we have

$$
\begin{aligned}
\epsilon_{2}(\beta) & =-\frac{\left\langle r^{2} e^{i 2 \phi}\right\rangle_{e}-4\langle\beta\rangle_{c}}{\left\langle r^{2}\right\rangle_{e}-\left|\left\langle r e^{i \phi}\right\rangle_{e}\right|^{2}}, \\
T^{\tau \tau} & =A e^{-\frac{r^{2}}{2 \sigma^{2}}\left(1+a_{2} \cos 2 \phi\right)} \\
|C| & =r^{2} P e^{-\frac{r^{2}}{2 \varrho^{2}}\left(1-p_{2} \cos 2 \phi\right)} \\
\phi_{c} & =\phi-q_{2} \sin 2 \phi .
\end{aligned}
$$

TABLE 4.5: Parameters for $V_{2} \times \epsilon_{2}(\beta)$.

\begin{tabular}{|c|c|c|c|}
\hline$P\left(\mathrm{fm}^{-6}\right)$ & $a_{2}$ & $p_{2}$ & $q_{2}$ \\
\hline \hline$\{0.0-10.0\}$ & $\{0.0-0.6\}$ & $\{0.0-0.4\}$ & $\{0.0-0.6\}$ \\
\hline
\end{tabular}

Each point in these scatters (Figure 4.8) represents a different event where we changed values for all parameters. It is possible to realize that for the first case which has $\beta=$ $0, V_{2}$ has points in vertical, that exist because the elliptic flow coefficient is sensitive to asymmetries from initial stress tensor, but with this value of $\beta=0$, the eccentricity does not capture these effects. On the other hand, when $\beta=2.0$ and $4.0 \mathrm{fm}, \epsilon_{2}$ captures these effects even there is not a linear relationship between $V_{2}$ and $\epsilon_{2}$, but it is possible to figure out the best value of $\beta$ where in Figure 4.8 it is possible to obtain a linear relationship. 

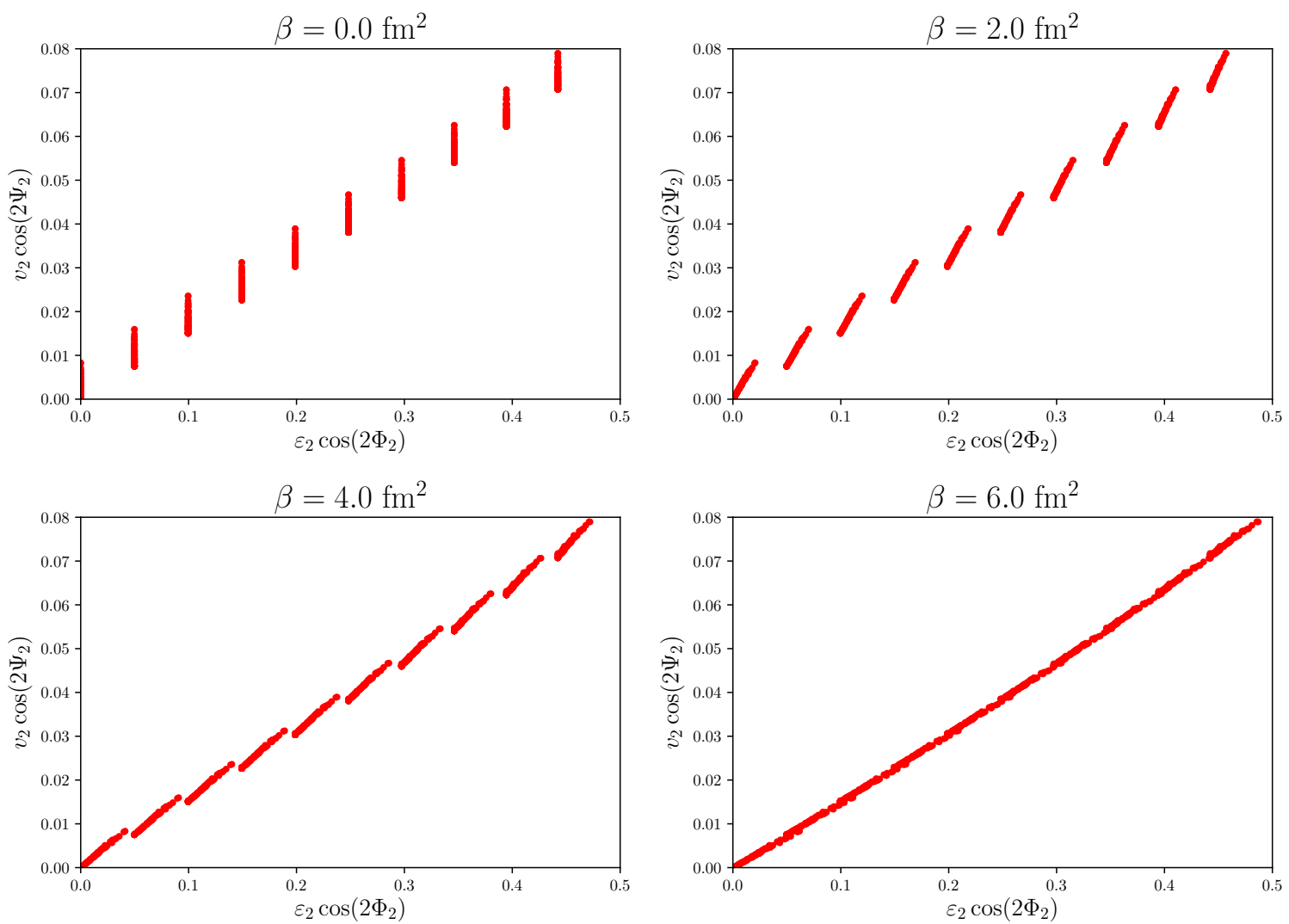

FiguRE 4.8: Plots of $V_{2} \times \epsilon_{2}(\beta=0)$ (upper left), of $V_{2} \times \epsilon_{2}(\beta=2.0) \mathrm{fm}$ (upper right), of $V_{2} \times \epsilon_{2}(\beta=4.0) \mathrm{fm}$ (lower left), and of $V_{2} \times \epsilon_{2}(\beta=6.0) \mathrm{fm}$ (lower right).

On the other case where $n=3$, using the best value of $\beta$, the behavior is similar, and we have

$$
\epsilon_{3}(\beta)=-\frac{\left\langle r^{3} e^{i 3 \phi}\right\rangle_{e}-12 \beta\left\langle r e^{i \phi}\right\rangle_{c}}{\left(\left\langle r^{2}\right\rangle_{e}-\left|\left\langle r e^{i \phi}\right\rangle_{e}\right|^{2}\right)^{\frac{3}{2}}} .
$$

And analogously as made with the parameters of $\epsilon_{2}$, for $\epsilon_{3}$, we have

TABLE 4.6: Parameters for $V_{3} \times \epsilon_{3}(\beta)$.

\begin{tabular}{|c|c|c|c|}
\hline$P\left(\mathrm{fm}^{-6}\right)$ & $a_{3}$ & $p_{3}$ & $q_{3}$ \\
\hline \hline$\{0.0-10.0\}$ & $\{0.0-0.6\}$ & $\{0.0-0.4\}$ & $\{0.0-0.6\}$ \\
\hline
\end{tabular}

After checking these results, it was clear that both cases are linear for the same value of $\beta=6.0 \mathrm{fm}^{2}$ proving that the framework from ansatz (4.30) works. 


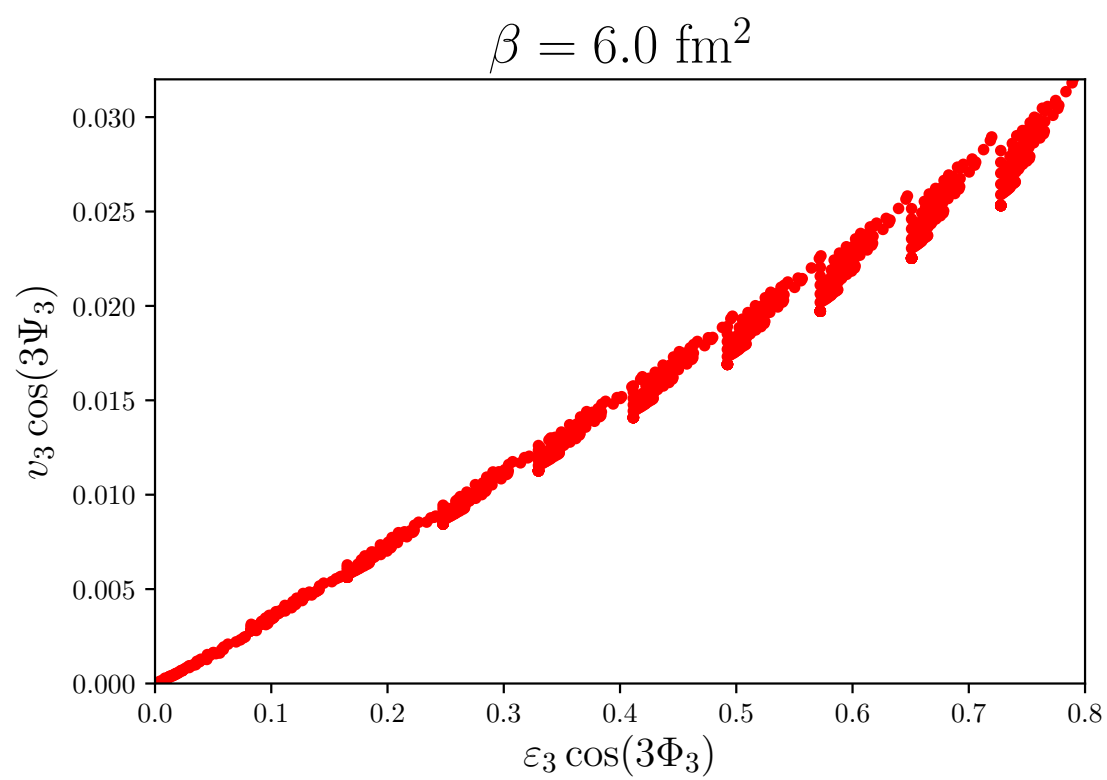

FIGURE 4.9: Relation between $V_{3}$ and $\epsilon_{3}(\beta)$.

\subsection{Both effects together}

This section is responsible by to include both contributions together. Basically, the procedure is exactly the same but now using the generating function from Equation (4.1) that in order to separate the scales we take Fourier transform as

$$
\rho(\vec{k})=\frac{1}{2 \pi} \int d^{2} x\left(T^{\tau \tau}-\alpha \partial_{i} T^{\tau i}-\beta \partial_{i} \partial_{j} T^{i j}\right) e^{i \vec{k} \cdot \vec{x}}
$$

and to select large-scales of system, it was taken Maclaurin series and to identify quantities with the correct rotational properties a Fourier expansion was performed

$$
\rho(\vec{x})=\sum_{m=0}^{\infty} \sum_{n=-\infty}^{\infty} W_{n, m}(\alpha, \beta) k^{m} e^{-i n \phi_{k}},
$$


with these equations, the general moment are trivially written as

$$
\begin{aligned}
\rho_{n, m}(\alpha, \beta)= & \frac{i^{m}}{2^{m}\left(\frac{m+n}{2}\right) !\left(\frac{m-n}{2}\right) !}\left[\int d^{2} x r^{m} e^{i n \phi} T^{\tau \tau}(\vec{x})\right. \\
& -\alpha\left(\frac{m+n}{2}\right) \int d^{2} x r^{m-1} e^{i(n-1) \phi} U(\vec{x}) \\
& -\alpha\left(\frac{m-n}{2}\right) \int d^{2} x r^{m-1} e^{i(n+1) \phi} U^{*}(\vec{x}) \\
& -\beta(m+n)\left(\frac{m+n}{2}-1\right) \int d^{2} x r^{m-2} e^{i(n-2) \phi} C(\vec{x}) \\
& -\beta(m-n)\left(\frac{m-n}{2}-1\right) \int d^{2} x r^{m-2} e^{i(n+2) \phi} C^{*}(\vec{x}) \\
& \left.-\beta(m+n)(m-n) \int d^{2} x r^{m-2} e^{i n \phi} \frac{T}{2}(\vec{x})\right],
\end{aligned}
$$

And now, in order to generate cumulants, the same idea of $W(\vec{k})$ was made such that the cumulants are

$$
\begin{gathered}
W_{0,2}(\alpha, \beta)=\frac{1}{2} \frac{i^{2}}{2 !}\left[\left\langle r^{2}\right\rangle_{e}-\alpha\left\langle r e^{-i \phi}\right\rangle_{u}-\alpha\left\langle r e^{i \phi}\right\rangle_{u^{*}}-4\langle\beta\rangle_{T}-\left|\left\langle r e^{i \phi}\right\rangle_{e}-\langle\alpha\rangle_{u}\right|^{2}\right] \\
W_{2,2}(\alpha, \beta)=\frac{1}{4} \frac{i^{2}}{2 !}\left[\left\langle r^{2} e^{i 2 \phi}\right\rangle_{e}-2 \alpha\left\langle r e^{i \phi}\right\rangle_{u}-4\langle\beta\rangle_{c}-\left(\left\langle r e^{i \phi}\right\rangle_{e}-\langle\alpha\rangle_{u}\right)^{2}\right] \\
W_{3,3}(\alpha, \beta)=\frac{1}{8} \frac{i^{3}}{3 !}\left[\left\langle r^{3} e^{i 3 \phi}\right\rangle_{e}-3 \alpha\left\langle r^{2} e^{i 2 \phi}\right\rangle_{u}-12 \beta\left\langle r e^{i \phi}\right\rangle_{c}-\left(\left\langle r e^{i \phi}\right\rangle_{e}-\langle\alpha\rangle_{u}\right)\right. \\
\left.\cdot\left(3\left\langle r^{2} e^{i 2 \phi}\right\rangle_{e}-6 \alpha\left\langle r e^{i \phi}\right\rangle_{u}-12\langle\beta\rangle_{c}-2\left(\left\langle r e^{i \phi}\right\rangle_{e}-\langle\alpha\rangle_{u}\right)^{2}\right)\right] .
\end{gathered}
$$

The total eccentricity can be defined as

$$
\epsilon_{n}(\alpha, \beta)=-n ! \frac{W_{n, n}(\alpha, \beta)}{\left(W_{0,2}^{e}\right)^{\frac{n}{2}}}
$$

where $\epsilon_{2}$ and $\epsilon_{3}$ are the eccentricities more important to check in simulations, thus we have

$$
\epsilon_{2}(\alpha, \beta)=-\frac{\left\langle r^{2} e^{i 2 \phi}\right\rangle_{e}-2 \alpha\left\langle r e^{i \phi}\right\rangle_{u}-4\langle\beta\rangle_{c}-\left(\left\langle r e^{i \phi}\right\rangle_{e}-\langle\alpha\rangle_{u}\right)^{2}}{\left\langle r^{2}\right\rangle_{e}-\left|\left\langle r e^{i \phi}\right\rangle_{e}\right|^{2}}
$$


as the same way, $\epsilon_{3}$ is

$$
\begin{aligned}
\epsilon_{3}(\alpha, \beta) & =-\frac{\left\langle r^{3} e^{i 3 \phi}\right\rangle_{e}-3 \alpha\left\langle r^{2} e^{i 2 \phi}\right\rangle_{u}-12 \beta\left\langle r e^{i \phi}\right\rangle_{c}}{\left(\left\langle r^{2}\right\rangle_{e}-\left|\left\langle r e^{i \phi}\right\rangle_{e}\right|^{2}\right)^{\frac{3}{2}}} \\
& -\frac{\left(\left\langle r e^{i \phi}\right\rangle_{e}-\langle\alpha\rangle_{u}\right)\left(3\left\langle r^{2} e^{i 2 \phi}\right\rangle_{e}-6 \alpha\left\langle r e^{i \phi}\right\rangle_{u}-12\langle\beta\rangle_{c}\right)-2\left(\left\langle r e^{i \phi}\right\rangle_{e}-\langle\alpha\rangle_{u}\right)^{3}}{\left(\left\langle r^{2}\right\rangle_{e}-\left|\left\langle r e^{i \phi}\right\rangle_{e}\right|^{2}\right)^{\frac{3}{2}}} .
\end{aligned}
$$

In order to check the relation between $V_{n}$ and $\epsilon_{n}(\alpha, \beta)$, we have Figure 4.10 plots events made for $n=2$ and in particular centered coordinate system such that $W_{1,1}(\alpha, \beta)=0$, we have

$$
\epsilon_{2}(\alpha, \beta)=-\frac{\left\langle r^{2} e^{i 2 \phi}\right\rangle_{e}-2 \alpha\left\langle r e^{i \phi}\right\rangle_{u}-4\langle\beta\rangle_{c}}{\left\langle r^{2}\right\rangle_{e}-\left|\left\langle r e^{i \phi}\right\rangle_{e}\right|^{2}}
$$

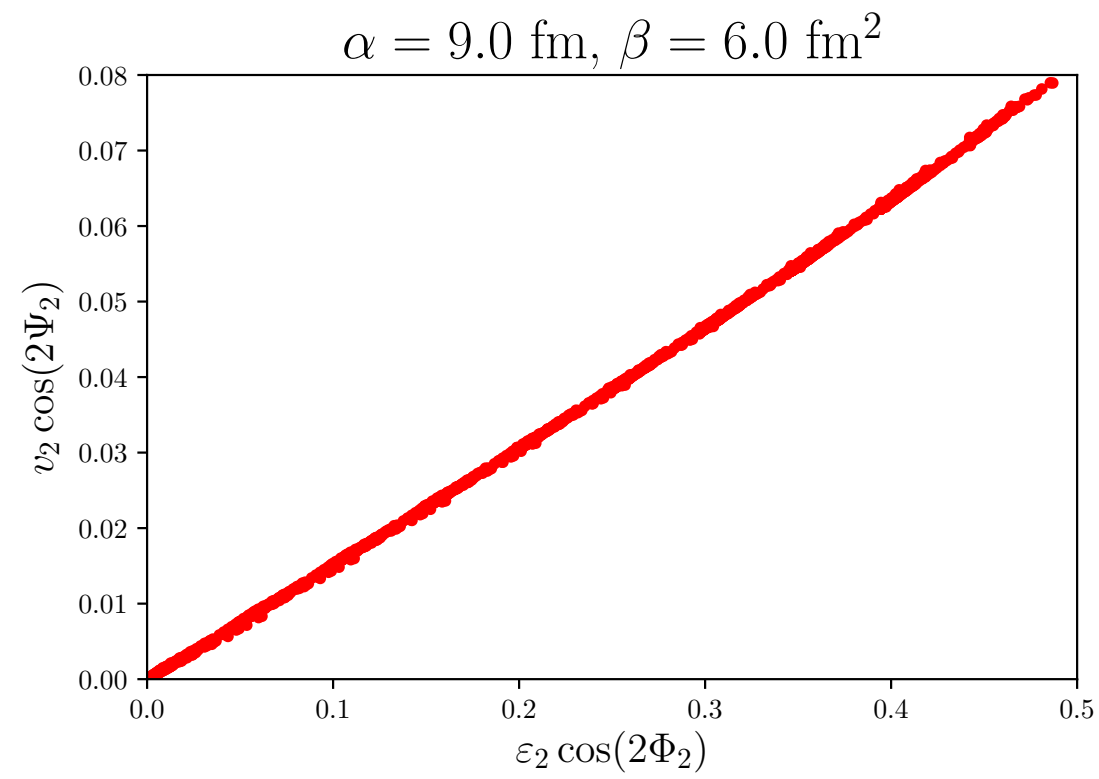

FIGURE 4.10: Relation between $V_{2}$ and $\epsilon_{2}(\alpha, \beta)$ event-by-event.

TABLE 4.7: Parameters used for $V_{2} \times \epsilon_{2}(\alpha, \beta)$.

\begin{tabular}{|c|c|c|c|}
\hline$B\left(\mathrm{fm}^{-5}\right)$ & $a_{2}$ & $b_{2}$ & $c_{2}$ \\
\hline \hline$\{0.0-10.0\}$ & $\{0.0-0.6\}$ & $\{0.0-0.6\}$ & $\{0.0-0.4\}$ \\
\hline$P\left(\mathrm{fm}^{-6}\right)$ & $p_{2}$ & $q_{2}$ \\
\hline \hline$\{0.0-10.0\}$ & $\{0.0-0.4\}$ & $\{0.0-0.6\}$ \\
\hline
\end{tabular}


On the other case where $n=3$, the behavior is really similar for a range of eccentricity between 0 and 0.4 the plot is linear for the same value of $\alpha$ and $\beta$.

$$
\epsilon_{3}(\alpha, \beta)=-\frac{\left\langle r^{3} e^{i 3 \phi}\right\rangle_{e}-3 \alpha\left\langle r^{2} e^{i 2 \phi}\right\rangle_{u}-12 \beta\left\langle r e^{i \phi}\right\rangle_{c}}{\left(\left\langle r^{2}\right\rangle_{e}-\left|\left\langle r e^{i \phi}\right\rangle_{e}\right|^{2}\right)^{\frac{3}{2}}} .
$$

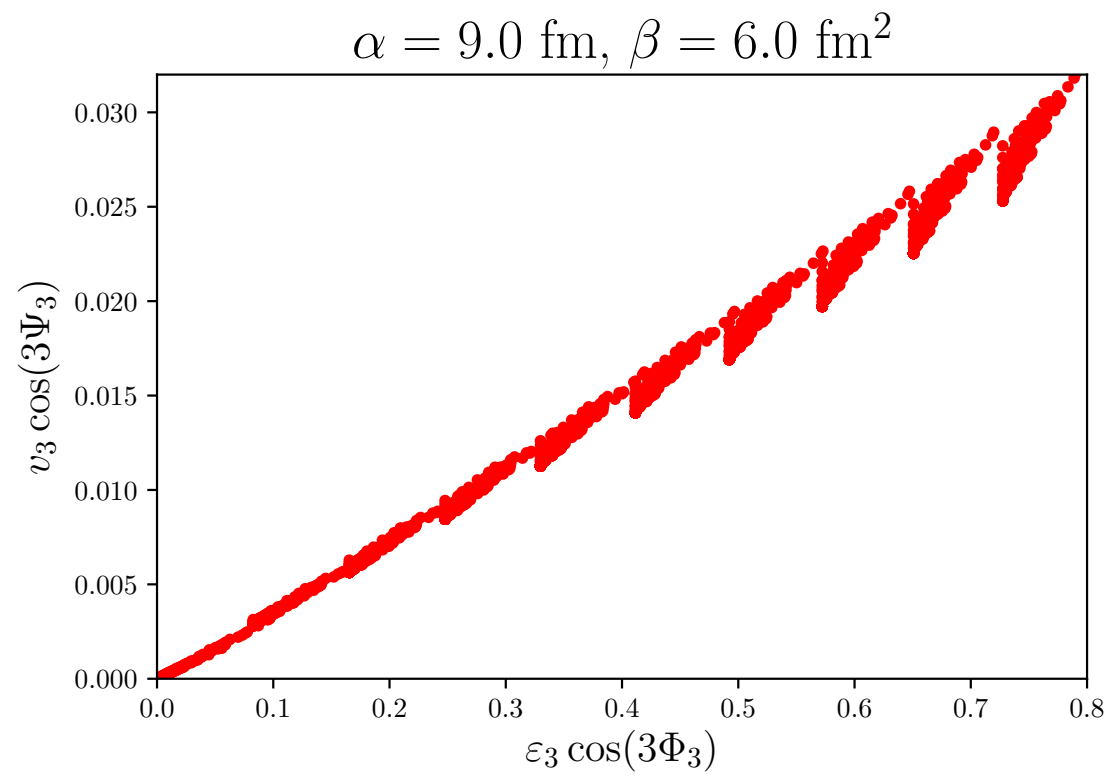

FIGURE 4.11: Relation between $V_{3}$ and $\epsilon_{3}(\alpha, \beta)$ event-by-event.

TABLE 4.8: Parameters used for $V_{3} \times \epsilon_{3}(\alpha, \beta)$.

\begin{tabular}{|c|c|c|c|}
\hline$B\left(\mathrm{fm}^{-5}\right)$ & $a_{3}$ & $b_{3}$ & $c_{3}$ \\
\hline \hline$\{0.0-10.0\}$ & $\{0.0-0.6\}$ & $\{0.0-0.6\}$ & $\{0.0-0.4\}$ \\
\hline$P\left(\mathrm{fm}^{-6}\right)$ & $p_{3}$ & $q_{3}$ \\
\hline \hline$\{0.0-10.0\}$ & $\{0.0-0.4\}$ & $\{0.0-0.6\}$ \\
\hline
\end{tabular}

After checking these results, it was clear that both are linear for the same values of $\alpha=9.0 \mathrm{fm}$ and $\beta=6.0 \mathrm{fm}^{2}$, showing that our ansatz (4.45) make sense. 


\section{Chapter 5}

\section{Realistic simulations}

This chapter focuses on analyzing simulations considered more realistic because their initial condition fit with experimental data. We perform state-of-the-art simulations using IPGlasma initial conditions of $\mathrm{Pb}+\mathrm{Pb}$ collisions for $2760 \mathrm{GeV}$ which is the same energy of LHC, viscous hydrodynamics, and UrQMD afterburner [61-63]. We take the fluid properties from a previous Bayesian analysis [71]. Note that because of these differences compared to the Toy Model calculations, we expect the response coefficients $\kappa_{n}, \alpha$ and $\beta$ to be different.

Any "estimator" for $V_{n}$ is necessarily an approximation. Thus, once a estimator $\epsilon_{n}$ is established, it is judged by how accurately it can predict $V_{n}$ on event-by-event basis. A given estimator must be an accurate estimation in particular specially-chosen event, but a poor estimation in other events. In other words, there are fluctuating initial conditions [72] and therefore, it is useful to define a measure of the quality of a proposed estimation that is preferably tested over a large and diverse set of realistic collision events.

The idea is identify whether the estimator $\left(\epsilon_{n}\right)$ with new contributions improve, and the natural choice to check is the linear correlation coefficient (Pearson coefficient) between the estimator and the final flow vector $V_{n}$ over the ensemble of events and as used at [36], the Pearson coefficient can be written as

$$
Q_{n}=\frac{R e\left\{V_{n} \epsilon_{n}^{*}\right\}}{\sqrt{\left\{\left|V_{n}\right|^{2}\right\}\left\{\left|\epsilon_{n}\right|^{2}\right\}}},
$$

where

$$
\{\ldots\}=\frac{1}{N_{\text {events }}} \sum_{\text {events }} \ldots
$$

Note that the numerator is an average of the scalar product of two vectors, and $Q_{n}$ shows how much these two vectors are linear. It is bounded by \pm 1 , with a value of 1 obtained if and only if the estimator gives a perfect prediction of $V_{n}$ in every event. In general, larger values (values closer to 1) indicate a better linear correlation and therefore a better estimator, and on other hand if values are close to zero, this coefficient indicates that the estimators do not have relation with the final state. 


\subsection{Usual estimator}

We can actually test this idea quantitatively by calculating a large set of events and computing the quantity estimator in Eq. (5.1), which in this case becomes explicitly for $n=2$ and 3 , respectively as

$$
Q_{2}=\frac{\left\{v_{2} \varepsilon_{2} \cos 2\left(\Psi_{2}-\Phi_{2}\right)\right\}}{\sqrt{\left\{v_{2}^{2}\right\}\left\{\varepsilon_{2}^{2}\right\}}},
$$

and

$$
Q_{3}=\frac{\left\{v_{3} \varepsilon_{3} \cos 3\left(\Psi_{3}-\Phi_{3}\right)\right\}}{\sqrt{\left\{v_{3}^{2}\right\}\left\{\varepsilon_{3}^{2}\right\}}} .
$$

The approach was in previous chapters to establish a relation between initial conditions and final state was made here, using Pearson coefficients, for the first time in [35]. The Equations (5.3) and (5.4) were calculated using only energy density contribution, these equations are indeed in a good approximation as a vector equation and it means that the event plane $\Psi_{n}$ is approximately the same as the participant plane $\Phi_{n}$ in each event, and consequently the elliptic flow $V_{2}$ is proportional to the eccentricity $\epsilon_{2}$ and in the same way, the triangular flow $V_{3}$ is also proportional to eccentricity $\epsilon_{3}$.

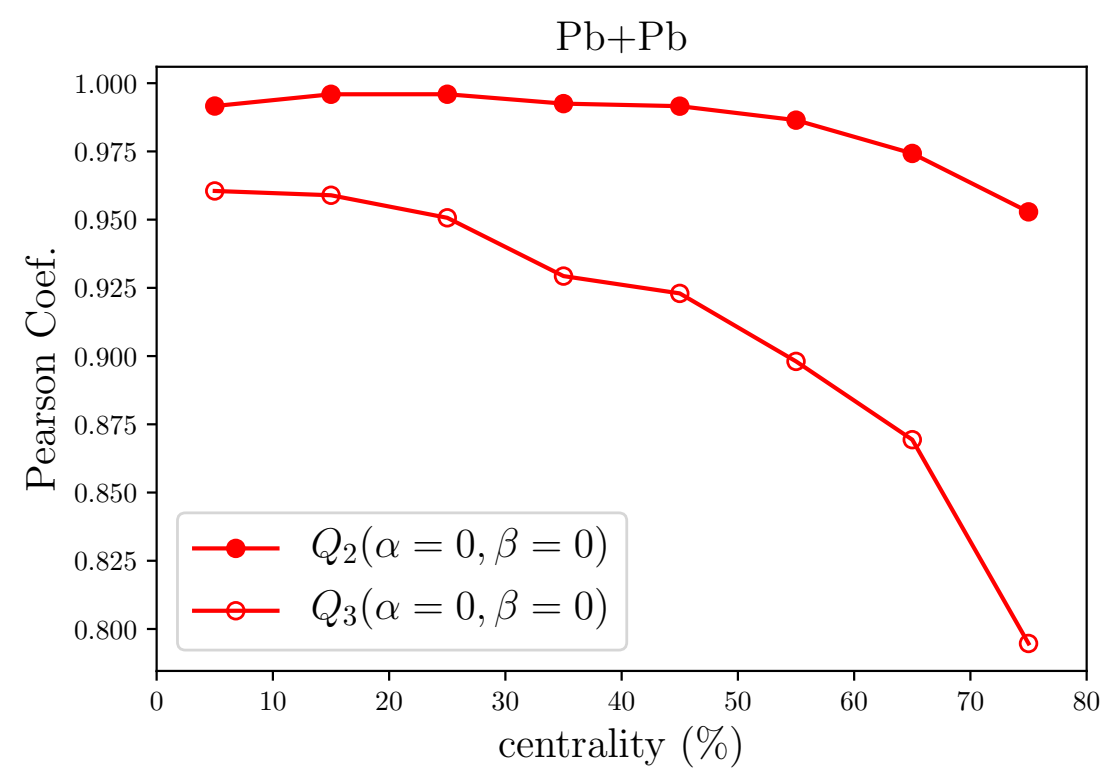

FIGURE 5.1: $Q_{n}(\alpha=0, \beta=0)$.

Figure 5.1 shows that only the contribution of energy density makes $Q_{2}$ and $Q_{3}$ close to 1 , which makes $\epsilon_{n}^{e}$ a good estimator. And although we already have a good estimator like it, we checked whether new contributions are able to improve the estimator even more.

In this approximation, the coefficient $\kappa_{n}$ from Eq. (3.27) contains all relevant information about properties such as viscosity, as well as freeze out and subsequent evolution the system, and this is the same in every collision event at a given centrality. Conversely the only thing that changes from one event to the next is the initial condition, and the only aspect that 
matters is the large scale structure, and all these characteristics for this initial conditions (IP-Glasma) are totally different than Toy Model. These large differences make the response coefficient $\kappa_{n}$ become totally different for both models.

The response coefficient $\kappa_{n}$ [36] can be written as

$$
\kappa_{n}=\frac{\operatorname{Re}\left\{V_{n} \epsilon_{n}^{*}\right\}}{\left\{\left|\epsilon_{n}\right|^{2}\right\}},
$$

where, figure 5.2 shows that the coefficient $\kappa_{n}$ does not change very much in different centralities.

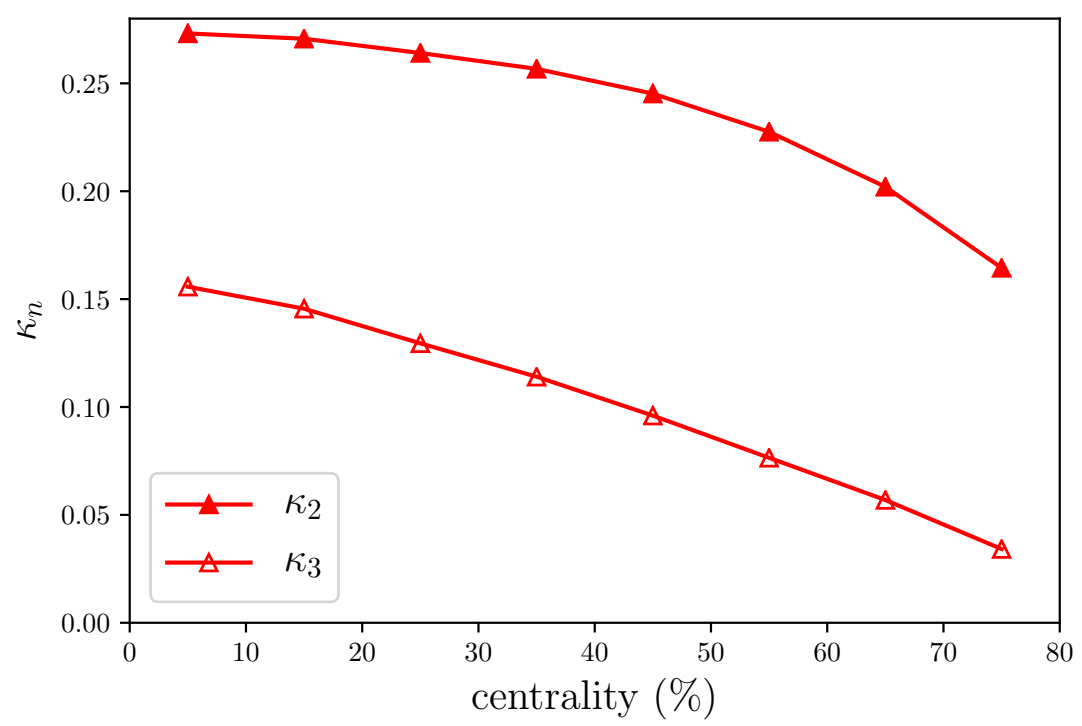

FIGURE 5.2: Response coefficient $\kappa_{n}$ for different centralities.

\subsection{Estimator with new contributions}

Now, we are interested to check if the estimator with new contributions improves when compared to estimator with energy density only. The Pearson coefficient as a function of $\alpha$ and $\beta$ was constructed, then we have

$$
Q_{n}(\alpha, \beta)=\frac{\operatorname{Re}\left\{V_{n} \epsilon_{n}^{*}(\alpha, \beta)\right\}}{\sqrt{\left\{\left|V_{n}\right|^{2}\right\}\left\{\left|\epsilon_{n}(\alpha, \beta)\right|^{2}\right\}}},
$$

where now, we checked its values for different values of $\alpha$ and $\beta$.

In order to identify the maximum value of $Q_{n}(\alpha, \beta)$ for each harmonic and in each centrality, its values were checked changing $\alpha$ and $\beta$, where the $\alpha$ and $\beta$ that make the Pearson coefficient maximum were called as "best values" and are available in tables 4.1 and 4.2. 
TABLE 5.1: Best values of $\alpha$ and $\beta$ for $n=2$.

\begin{tabular}{|c|c|c|}
\hline Centrality & $\alpha_{\text {best }}(\mathrm{fm})$ & $\beta_{\text {best }}\left(\mathrm{fm}^{2}\right)$ \\
\hline \hline $0-10 \%$ & 5.5 & 31.5 \\
\hline $10-20 \%$ & 4.5 & 26.5 \\
\hline $20-30 \%$ & 3.5 & 23.0 \\
\hline $30-40 \%$ & 3.0 & 20.5 \\
\hline $40-50 \%$ & 2.5 & 18.5 \\
\hline $50-60 \%$ & 2.0 & 15.5 \\
\hline $60-70 \%$ & 1.5 & 13.0 \\
\hline $70-80 \%$ & 0.5 & 9.0 \\
\hline $80-90 \%$ & 0.5 & 6.0 \\
\hline $90-100 \%$ & 1.0 & 6.5 \\
\hline
\end{tabular}

TABLE 5.2: Best values of $\alpha$ and $\beta$ for $n=3$.

\begin{tabular}{|c|c|c|}
\hline Centrality & $\alpha_{\text {best }}(\mathrm{fm})$ & $\beta_{\text {best }}\left(\mathrm{fm}^{2}\right)$ \\
\hline \hline $0-10 \%$ & 0.0 & 26.0 \\
\hline $10-20 \%$ & 0.0 & 22.0 \\
\hline $20-30 \%$ & 0.0 & 20.5 \\
\hline $30-40 \%$ & 0.0 & 20.0 \\
\hline $40-50 \%$ & 0.0 & 19.0 \\
\hline $50-60 \%$ & 0.0 & 17.0 \\
\hline $60-70 \%$ & 0.0 & 14.5 \\
\hline $70-80 \%$ & 0.0 & 12.0 \\
\hline $80-90 \%$ & 0.0 & 12.5 \\
\hline $90-100 \%$ & 0.0 & 12.5 \\
\hline
\end{tabular}

Comparing the Pearson coefficient that has only the energy density contributing $(\alpha=0$, $\beta=0$ ) with itself using the best values of $\alpha$ and $\beta$, we have in Figure 4.3 the improvement maximum for each harmonic in each centrality.

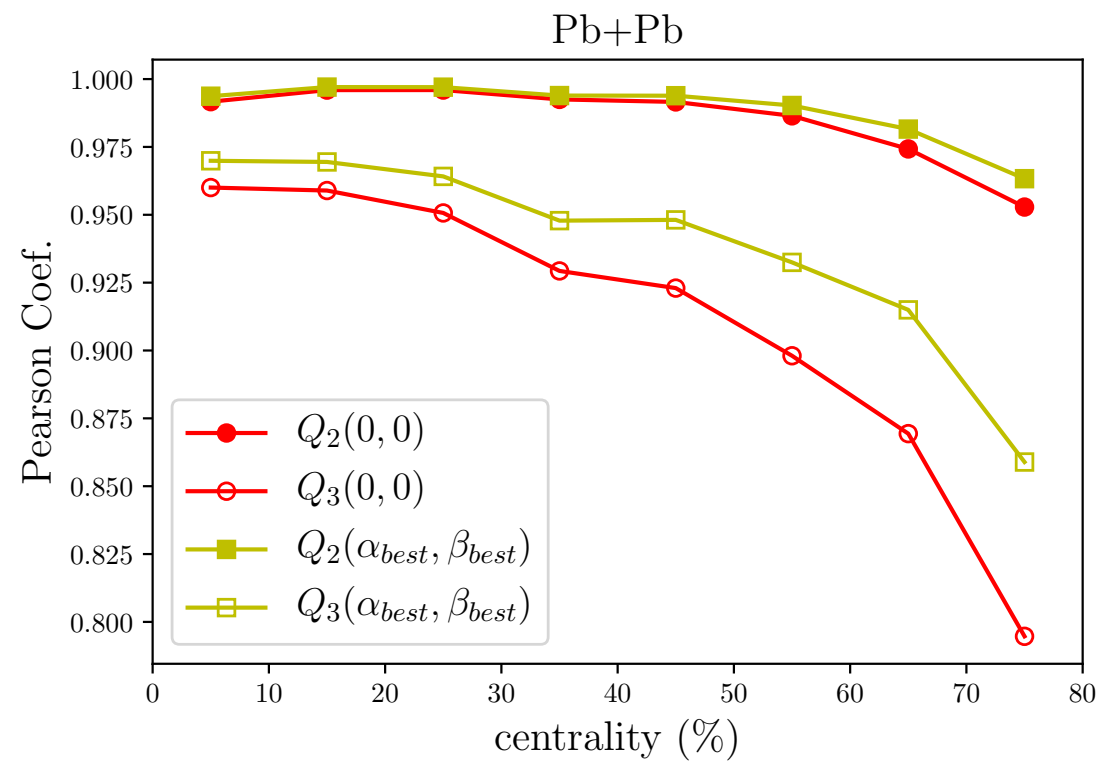

FIGURE 5.3: $Q_{n}(0,0)$, and $Q_{n}\left(\alpha_{\text {best }}, \beta_{\text {best }}\right)$.

This improvement is not large but it exists, showing that it is possible makes an estimator able to capture other $T^{\mu \nu}$ effects.

We know that in the Chapter 4, $\alpha$ and $\beta$ were defined as independent of harmonic, but in Tables 5.1 and 5.2 they are different. Then, an important issue to discuss is whether these differences are significant or negligible. In order to answer this issue, we can choose single values of $\alpha$ and $\beta$ for $Q_{n}$, compare with $Q_{n}\left(\alpha_{\text {best }}, \beta_{\text {best }}\right)$, and check whether there are 
significant differences between them. We indeed find that estimators of equivalent quality can be obtained with single values, independent of harmonic.

To make an even stronger test, in Figure 5.4 we show the result for a single value of $\alpha$ and $\beta$ for all centralities as well as harmonics. While the response coefficients are allowed to depend on centrality, they typically have a relatively weak dependence (see Figure 5.2). We see that a single value of $\alpha=2.0 \mathrm{fm}$, and $\beta=20.0 \mathrm{fm}^{2}$ gives an estimator of equivalent quality, until $\sim 60 \%$ centrality, above which smaller values of $\alpha$ and $\beta$ are favored.

This constancy of response coefficients gives strong evidence for our non-trivial ansatz and validates our framework, which indeed captures these new effects of the initial conditions of the system in an accurate way.

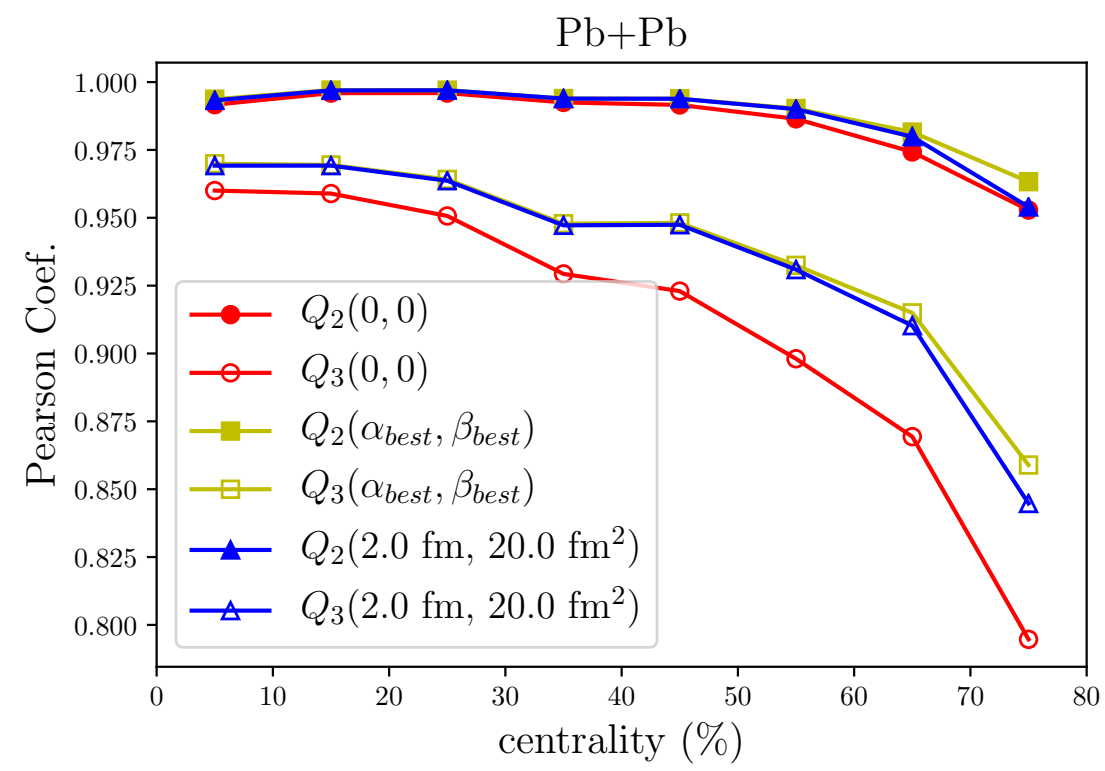

FIGURE 5.4: $Q_{n}(0,0), Q_{n}\left(\alpha_{\text {best }}, \beta_{\text {best }}\right)$, and $Q_{n}\left(\alpha=2.0 \mathrm{fm}, \beta=20.0 \mathrm{fm}^{2}\right)$. 


\section{Chapter 6}

\section{Conclusions}

The field that study relativistic heavy-ion collisions deals with events where the nuclei are imparted with high-energy. This energy is used to break up the nuclei, making possible to obtain a system that consists of a "soup" of quarks and gluons. When this system evolves a extreme high temperature, it is possible to create a new state of the matter called quark-gluon plasma. This state behaviors as a relativistic fluid, and hence respect properties of relativistic hydrodynamics. Under these properties, it is possible to establish a relationship between initial condition represented by the familiar eccentricities $\epsilon_{n}$ and the final state represented by the harmonic flow coefficients $V_{n}$.

In this thesis, we perform hydrodynamic response to initial conditions of only energy density event-by-event and have confirmed the efficacy of this response. In order to include effects from others $T^{\mu \nu}$ components, an ansatz (3.1) able to make these inclusions was constructed. Specifically, we included effects from initial momentum density $T^{\tau i}$ and initial stress tensor $T^{i j}$. This ansatz has two response coefficients (one for each new contribution) and we have perform hydrodynamic simulations using the Toy Model to check whether the new eccentricities make sense and whether this framework works.

Initially, the initial conditions used in Toy Model do not represent a realistic collision, but they contain enough information to confirm that the ansatz makes sense and works. On the other moment, IP-Glasma initial conditions were also performed in order to give validation to our ansatz. For these initial conditions, we figured out constant values of $\alpha$ and $\beta$ that improve the estimator $\epsilon_{n}(\alpha, \beta)$ compared with the usual eccentricity that depends only of energy density $\epsilon_{n}(\alpha=0, \beta=0)$. We realized that $\alpha$ and $\beta$ behavior similarly to $\kappa_{n}$ in different centralities. Therefore, it was possible to establish constant values of $\alpha$ and $\beta$ for both harmonics in different centralities that maximize the Pearson coefficient, which makes possible to give validation to ansatz.

In general, the idea these new contributions was checked and it was possible to make these inclusions. In the future we will perform simulations using smaller collision systems where the new effects can increase their importance.

Some questions that can be raised are the nature of the response coefficients $\alpha$ and $\beta$, what can interfere them, whether it is possible to expand this improvement for all harmonics, whether other terms of initial energy-momentum tensor can also be included, and further improve what has already been done. They are questions prospects works which can be made in the future. 


\section{Appendix A}

\section{Derivation of cumulants}

In order to develop the derivation of cumulants with more detail, this appendix was made in first place, Taking a (2-D) Fourier transform of a generating function, we have

$$
\rho(\vec{k})=\frac{1}{2 \pi} \int d^{2} x \rho(\vec{x}) e^{i \vec{k} \cdot \vec{x}}
$$

Expanding in Maclaurin series give us

$$
\rho(\vec{k}) \equiv \sum_{m=0}^{\infty} \rho_{m}\left(\phi_{k}\right) k^{m},
$$

and soon later, we expand in Fourier series and arrive at next expression

$$
\rho(\vec{k}) \equiv \sum_{n=-\infty}^{\infty} \sum_{m=0}^{\infty} \rho_{n, m} k^{m} e^{-i n \phi_{k}} .
$$

In order to define the cumulants, we set $W(\vec{k})$ from

$$
\rho(\vec{k}) \equiv e^{W(\vec{k})}
$$

And as the same way we expand it in Maclaurin series

$$
W(\vec{k}) \equiv \sum_{m=0}^{\infty} W_{m} k^{m}
$$

and in Fourier series

$$
W(\vec{k}) \equiv \sum_{m=0}^{\infty} \sum_{n=-\infty}^{\infty} W_{n, m} k^{m} e^{-i n \phi_{k}}
$$


We start by expanding the Fourier transform in powers of $k$.

$$
\begin{aligned}
\rho(\vec{k}) & =\frac{1}{2 \pi} \int d^{2} x \rho(\vec{x}) \sum_{m=0}^{\infty} \frac{1}{m !}(i \vec{k} \cdot \vec{x})^{m}, \\
& =\sum_{m=0}^{\infty} k^{m} \frac{i^{m}}{m !} \int d^{2} x r^{m} \rho(\vec{x}) \cos ^{m}\left(\phi_{k}-\phi\right) .
\end{aligned}
$$

Matching the appropriate power of $k$ gives

$$
\rho_{m}(\vec{k})=\frac{i^{m}}{m !} \int d^{2} x r^{m} \rho(\vec{x}) \cos ^{m}\left(\phi_{k}-\phi\right) .
$$

Projecting out the nth Fourier harmonic gives

$$
\begin{aligned}
\rho_{n, m} & =\frac{1}{2 \pi} \int d^{2} x \rho\left(\phi_{k}\right) e^{i n \phi_{k}} \\
& =\frac{1}{2 \pi} \frac{i^{m}}{m !} \int d^{2} x r^{m} \rho(\vec{x}) \cos ^{m}\left(\phi_{k}-\phi\right) e^{i n \phi_{k}} \\
& =\frac{1}{2 \pi} \frac{i^{m}}{m !} \int d \phi_{k} \int d^{2} x r^{m} \rho(\vec{x}) \cos ^{m}\left(\phi_{k}\right) e^{i n\left(\phi_{k}+\phi\right)} \\
& =\frac{1}{2 \pi} \frac{i^{m}}{m !} \int d \phi_{k} \cos ^{m}\left(\phi_{k}\right) e^{i n \phi_{k}} \int d^{2} x r^{m} \rho(\vec{x}) e^{i n \phi} \\
& =\frac{1}{2 \pi} \frac{i^{m}}{m !} 2 \pi\left(\begin{array}{c}
m \\
\frac{m+n}{2}
\end{array}\right) \int d^{2} x r^{m} \rho(\vec{x}) e^{i n \phi} \\
& =\frac{i^{m}}{\not n !} \frac{\not n !}{2^{m}\left(\frac{m+n}{2}\right) !\left(\frac{m-n}{2}\right) !} \int d^{2} x r^{m} e^{i n \phi} \rho(\vec{x}) \\
& =\frac{i^{m}}{2^{m}\left(\frac{m+n}{2}\right) !\left(\frac{m-n}{2}\right) !} \int d^{2} x r^{m} e^{i n \phi} \rho(\vec{x}) .
\end{aligned}
$$

The $\phi_{k}$ integral evaluated in the Eq. (A.9) is zero any time that $m<|n|$, or if $m-|n|$ is not an even number, and otherwise is given expression. 
The cumulants can then be written in terms of the moments. The moment expansion in terms of cumulants reads

$$
\begin{aligned}
\rho(\vec{k})= & e^{W(0)}\left[1+k W^{\prime}(0)\right. \\
& +\frac{1}{2 !} k^{2}\left(W^{\prime \prime}(0)+W^{\prime}(0)^{2}\right) \\
& \left.+\frac{1}{3 !} k^{3}\left(W^{(3)}(0)+W^{\prime}(0)^{3}+3 W^{\prime}(0) W^{\prime \prime}(0)\right)+O\left(k^{4}\right)\right] \\
& =\sum_{m=0}^{\infty} \rho_{m}\left(\phi_{k}\right) k^{m}=\sum_{m=0}^{\infty} \sum_{n=-m}^{m} \rho_{n, m} k^{m} e^{-i n \phi_{k}} .
\end{aligned}
$$

Matching powers of $k$ gives

- power 0:

$$
\begin{gathered}
\rho_{0}=e^{W(0)} . \\
W_{0,0}=\ln \left(\rho_{0,0}\right)
\end{gathered}
$$

- power 1:

$$
\begin{gathered}
\frac{\rho_{1}}{\rho_{0}}=\sum_{n} \frac{\rho_{n, 1}}{\rho_{0}} e^{i n \phi_{k}}=W^{\prime}(0)=\sum_{n} W_{n, 1} e^{i n \phi_{k}} \\
W_{1,1}=\frac{\rho_{1,1}}{\rho_{0,0}} \\
W_{-1,1}=\frac{\rho_{-1,1}}{\rho_{0,0}} .
\end{gathered}
$$

- power 2:

$$
\begin{aligned}
\frac{\rho_{2}}{\rho_{0}}=\sum_{n} \frac{\rho_{n, 1}}{\rho_{0}} e^{i n \phi_{k}}=\frac{1}{2}\left(W^{\prime \prime}(0)+W^{\prime}(0)^{2}\right) \\
=W_{0,2}+W_{-1,1} W_{1,1}+\left(\frac{1}{2} W_{1,1}^{2}+W_{2,2}\right) e^{i 2 \phi_{k}}+\left(\frac{1}{2} W_{-1,1}^{2}+W_{-2,2}\right) e^{-i 2 \phi_{k}} \\
W_{0,2}=\frac{\rho_{0,2}}{\rho_{0,0}}-W_{1,1} W_{-1,1} \\
W_{2,2}=\frac{\rho_{2,2}}{\rho_{0,0}}-W_{1,1}^{2} \\
W_{-2,2}=\frac{\rho_{-2,2}}{\rho_{0,0}}-W_{-1,1}^{2}
\end{aligned}
$$


- power 3:

$$
\begin{gathered}
\frac{\rho_{3}}{\rho_{0}}=\sum_{n} \frac{\rho_{n, 3}}{\rho_{0}} e^{i n \rho_{k}}=\frac{1}{6}\left(W^{\prime \prime \prime}(0)+W^{\prime}(0)^{3}+3 W^{\prime}(0) W^{\prime \prime}(0)\right) \\
=\left[W_{1,3}+W_{-1,1} W_{2,2}+W_{1,1} W_{0,2}+\frac{1}{2} W_{1,1}^{2} W_{-1,1}\right] e^{i \phi_{k}} \\
+\left[W_{3,3}+W_{1,1} W_{2,2}+\frac{1}{6} W_{1,1}^{3}\right] e^{i 3 \phi_{k}} \\
+\left[W_{-1,3}+W_{1,1} W_{-2,2}+W_{-1,1} W_{0,2}+\frac{1}{2} W_{-1,1}^{2} W_{-1,1}\right] e^{i \phi_{k}} \\
+\left[W_{-3,3}+W_{-1,1} W_{-2,2}+\frac{1}{6} W_{-1,1}^{3}\right] e^{i 3 \phi_{k}} \cdot \\
W_{1,3}=\frac{\rho_{1,3}}{\rho_{0,0}}-W_{2,2} W_{-1,1}-W_{1,1} W_{0,2}, \\
W_{-1,3}=\frac{\rho_{-1,3}}{\rho_{0,0}}-W_{-2,2} W_{1,1}-W_{-1,1} W_{0,2}, \\
W_{3,3}=\frac{\rho_{3,3}}{\rho_{0,0}}-W_{1,1} W_{2,2}-W_{1,1}^{3} \\
W_{-3,3}=\frac{\rho_{-3,3}}{\rho_{0,0}}-W_{-1,1} W_{-2,2}-W_{-1,1}^{3} .
\end{gathered}
$$

Finally, matching each azimuthal harmonics at each order, and substituting lower order solutions into the higher order equations:

$$
\begin{gathered}
W_{0,0}=\ln [\rho(\vec{k}=\overrightarrow{0})], \\
W_{1,1}=\frac{i}{2}\left\langle r e^{i \phi}\right\rangle, \\
W_{-1,1}=\frac{i}{2}\left\langle r e^{-i \phi}\right\rangle, \\
W_{0,2}=\frac{1}{2} \frac{i^{2}}{2 !}\left[\left\langle r^{2}\right\rangle-\left|\left\langle r e^{i \phi}\right\rangle\right|^{2}\right], \\
W_{2,2}=\frac{1}{4} \frac{i^{2}}{2 !}\left[\left\langle r^{2} e^{i 2 \phi}\right\rangle-\left\langle r e^{i \phi}\right\rangle^{2}\right], \\
W_{-2,2}=\frac{1}{4} \frac{i^{2}}{2 !}\left[\left\langle r^{2} e^{-i 2 \phi}\right\rangle-\left\langle r e^{-i \phi}\right\rangle^{2}\right],
\end{gathered}
$$




$$
\begin{gathered}
W_{1,3}=\frac{1}{8} \frac{i^{3}}{2 !}\left[\left\langle r^{3} e^{i \phi}\right\rangle-\left\langle r^{2} e^{i 2 \phi}\right\rangle\left\langle r e^{-i \phi}\right\rangle\right. \\
\left.-2\left\langle r^{2}\right\rangle\left\langle r e^{i \phi}\right\rangle+2\left\langle r e^{i \phi}\right\rangle^{2}\left\langle r e^{-i \phi}\right\rangle\right], \\
W_{-1,3}=\frac{1}{8} \frac{i^{3}}{2 !}\left[\left\langle r^{3} e^{-i \phi}\right\rangle-\left\langle r^{2} e^{-i 2 \phi}\right\rangle\left\langle r e^{i \phi}\right\rangle\right. \\
\left.-2\left\langle r^{2}\right\rangle\left\langle r e^{-i \phi}\right\rangle+2\left\langle r e^{-i \phi}\right\rangle{ }^{2}\left\langle r e^{i \phi}\right\rangle\right], \\
W_{3,3}=\frac{1}{8} \frac{i^{3}}{3 !}\left[\left\langle r^{3} e^{i 3 \phi}\right\rangle-\left\langle r e^{i \phi}\right\rangle\left(3\left\langle r^{2} e^{i 2 \phi}\right\rangle-2\left\langle r e^{i \phi}\right\rangle^{2}\right)\right], \\
W_{-3,3}=\frac{1}{8} \frac{i^{3}}{3 !}\left[\left\langle r^{3} e^{-i 3 \phi}\right\rangle-\left\langle r e^{-i \phi}\right\rangle\left(3\left\langle r^{2} e^{-i 2 \phi}\right\rangle-2\left\langle r e^{-i \phi}\right\rangle^{2}\right)\right] .
\end{gathered}
$$




\section{Appendix B}

\section{Developing the generating function}

\section{B.1 Including momentum density}

The generating function able to include effects of momentum density is

$$
\rho(\vec{x})=T^{\tau \tau}(\vec{x})+\alpha \partial_{i} T^{\tau i}(\vec{x}) .
$$

Let us take a Fourier Fourier transform

$$
\rho(\vec{k})=\frac{1}{2 \pi} \int d^{2} x[T^{\tau \tau}+\alpha \underbrace{\partial_{i} T^{\tau i}}_{\mathrm{I}}] e^{i \vec{k} \cdot \vec{x}} .
$$

Solving only the term I which is possible by parts, we have

$$
\begin{gathered}
\mathrm{I}=\int \overbrace{d^{2} x \partial_{i} T^{\tau i}(\vec{x})}^{d v} \overbrace{e^{i \vec{k} \cdot \vec{x}}}^{u} \\
=u v-\int v d u \\
=\left.T^{\tau x} e^{i \vec{k} \cdot \vec{x}}\right|_{-\infty} ^{+\infty}+\left.i T^{\tau y} e^{i \vec{k} \cdot x}\right|_{-\infty} ^{+\infty}-\int d^{2} x i k_{i} T^{\tau i} e^{i \vec{k} \cdot \vec{x}} . \\
\quad \mathrm{I}=-\int d^{2} x i k_{i} T^{\tau i} e^{i \vec{k} \cdot \vec{x}} . \\
=\rho_{0}-\alpha \rho_{1} .
\end{gathered}
$$

Now, expanding in Maclaurin and Fourier series, we have

$$
\rho(\vec{k})=\sum_{m=0}^{\infty} \sum_{|n| \leq m}^{m} \rho_{n, m} k^{m} e^{-i \phi_{k}} .
$$


We starting by expanding the Fourier transform in powers of $k$.

$$
\begin{aligned}
\rho(\vec{k}) & =\frac{1}{2 \pi} \int d^{2} x \sum_{m=0}^{\infty} \frac{1}{m !}(i \vec{k} \cdot \vec{x})^{m}\left[T^{\tau \tau}+i \alpha k_{i} T^{\tau i}\right] \\
& =\sum_{m=0}^{\infty} k^{m} \frac{1}{2 \pi} \frac{i^{m}}{m !} \int d^{2} x r^{m} \cos ^{m}\left(\phi_{k}-\phi\right)\left[T^{\tau \tau}+i k \alpha|U| \cos \left(\phi_{k}-\phi_{u}\right)\right]
\end{aligned}
$$

The $k^{0}$ term is identical to the $\alpha \rightarrow 0$ case.

$$
\begin{aligned}
\rho(\vec{k})=\rho_{0}+\sum_{m=1}^{\infty} k^{m} & {\left[\frac{i^{m}}{m !} \int d^{2} x r^{m} \cos ^{m}\left(\phi_{k}-\phi\right) T^{\tau \tau}\right.} \\
& \left.i \alpha \frac{i^{m-1}}{(m-1) !} \int d^{2} x|U| r^{m-1} \cos ^{m-1}\left(\phi_{k}-\phi\right) \cos \left(\phi_{k}-\phi_{u}\right)\right] .
\end{aligned}
$$

Matching the appropriate power of $k$ gives

$$
\begin{aligned}
\rho_{m}\left(\rho_{k}\right) & =\frac{i^{m}}{m !} \int d^{2} x r^{m} T^{\tau \tau} \cos ^{m}\left(\phi_{k}-\phi\right) \\
& -\alpha \frac{i^{m}}{(m-1) !} \int d^{2} x|U| r^{m-1} \cos ^{m-1}\left(\phi_{k}-\phi\right) \cos \left(\phi_{k}-\phi_{u}\right) .
\end{aligned}
$$

Projecting out the nth Fourier transform hasmonic gives

$$
\begin{gathered}
\rho_{n, m}=\int d \phi_{k} \rho_{m}\left(\rho_{k}\right) e^{i n \phi} \\
=\rho_{n, m}^{e}+\alpha \rho_{n, m}^{u}, \\
\rho_{n, m}^{e}=\frac{i^{m}}{2^{m}\left(\frac{m+n}{2}\right) !\left(\frac{m-n}{2}\right) !} \int d^{2} x r^{m} e^{i n \phi} T^{\tau \tau}, \\
\rho_{n, m}^{u}=\frac{i^{m}}{(m-1) !} \frac{1}{2 \pi} \int d \phi_{k} d^{2} x r^{m-1}|U| \cos ^{m-1}\left(\phi_{k}-\phi\right) \cos \left(\phi_{k}-\phi_{u}\right) e^{i n \phi_{k}} \\
=\frac{i^{m}}{2 \pi(m-1) !} \int d \phi_{k} d^{2} x r^{m-1}|U| \cos ^{m-1} \phi_{k} \cos \left(\phi_{k}-\phi_{u}+\phi\right) e^{i n\left(\phi_{k}+\phi\right)} \\
=\frac{i^{m}}{2 \pi(m-1) !} \int d \phi_{k} d^{2} x r^{m-1}|U| \cos ^{m-1} \phi_{k} \frac{1}{2}\left[e^{i\left(n \phi_{k}+n \phi+\phi_{k}-\phi_{u}+\phi\right)}\right. \\
\left.+e^{i\left(n \phi_{k}+n \phi-\phi_{k}+\phi_{u}-\phi\right)}\right] e^{i n\left(\phi_{k}+\phi\right)} \\
=\frac{i^{m}}{2 \pi(m-1) !} \int d \phi_{k} d^{2} x r^{m-1}|U| \cos ^{m-1} \phi_{k} \frac{1}{2}\left[e^{i(n+1) \phi_{k}} e^{\left.i\left([n+1] \phi-\phi_{u}\right)\right)}\right. \\
\left.+e^{i(n-1) \phi_{k}} e^{i\left([n-1] \phi+\phi_{u}\right)}\right],
\end{gathered}
$$




$$
\begin{aligned}
& \rho_{n, m}^{u}=\frac{1}{2} \frac{i^{m}}{2 \pi(m-1) !} \int d^{2} x r^{m-1}|U| {\left[\frac{2 \pi}{2^{m-1}}\left(\begin{array}{c}
m-1 \\
\frac{m+n}{2}
\end{array}\right) e^{i\left([n+1] \phi-\phi_{u}\right)}\right.} \\
&\left.\frac{2 \pi}{2^{m-1}}\left(\begin{array}{c}
m-1 \\
\frac{m+n-2}{2}
\end{array}\right) e^{i\left([n-1] \phi+\phi_{u}\right)}\right], \\
& \rho_{n, m}^{u}=\frac{1}{2} \frac{i^{m}}{(m-1) !} \int d^{2} x r^{m-1}|U| {\left[\frac{(m-1) !}{2^{m-1}\left(\frac{m+n}{2}\right) !\left(\frac{m-n-2}{2}\right) !} e^{i\left([n+1] \phi-\phi_{u}\right)}\right.} \\
&\left.+\frac{(m-1) !}{2^{m-1}\left(\frac{m+n-2}{2}\right) !\left(\frac{m-n}{2}\right) !} e^{i\left([n-1] \phi+\phi_{u}\right)}\right],
\end{aligned}
$$

and finally $\rho^{u}(\alpha)_{n, m}$ is

$$
\begin{aligned}
\rho_{n, m}^{u}=\frac{i^{m}}{2^{m}\left(\frac{m+n}{2}\right) !\left(\frac{m-n}{2}\right) !} \int d^{2} x r^{m-1} & {\left[\left(\frac{m-n}{2}\right) e^{i([n+1] \phi)} U^{*}\right.} \\
& \left.+\left(\frac{m+n}{2}\right) e^{i([n-1] \phi)} U\right],
\end{aligned}
$$

and hence the general moment is

$$
\begin{aligned}
\rho_{n, m}=\frac{i^{m}}{2^{m}\left(\frac{m+n}{2}\right) !\left(\frac{m-n}{2}\right) !}[ & \int d^{2} x r^{m} e^{i n \phi} T^{\tau \tau} \\
& -\alpha\left(\frac{m+n}{2}\right) \int d^{2} x r^{m-1} e^{i(n-1) \phi} U \\
& \left.-\alpha\left(\frac{m-n}{2}\right) \int d^{2} x r^{m-1} e^{i(n+1) \phi} U^{*}\right] .
\end{aligned}
$$

Similarly as made in Appendix A the cumulants are

$$
\begin{gathered}
W_{1,1}(\alpha)=\frac{i}{2}\left[\left\langle r e^{i \phi}\right\rangle_{e}-\langle\alpha\rangle_{u}\right], \\
W_{0,2}(\alpha)=\frac{1}{2} \frac{i^{2}}{2 !}\left[\left\langle r^{2}\right\rangle_{e}-\alpha\left\langle r e^{-i \phi}\right\rangle_{u}-\alpha\left\langle r e^{i \phi}\right\rangle_{u^{*}}-\left|\left\langle r e^{i \phi}\right\rangle_{e}-\langle\alpha\rangle_{u}\right|^{2}\right], \\
W_{2,2}(\alpha)=\frac{1}{4} \frac{i^{2}}{2 !}\left[\left\langle r^{2} e^{i 2 \phi}\right\rangle_{e}-2 \alpha\left\langle r e^{i \phi}\right\rangle_{u}-\left(\left\langle r e^{i \phi}\right\rangle_{e}-\langle\alpha\rangle_{u}\right)^{2}\right],
\end{gathered}
$$




$$
\begin{aligned}
W_{3,3}(\alpha)=\frac{1}{8} \frac{i^{3}}{3 !}\left[\left\langle r^{3} e^{i 3 \phi}\right\rangle_{e}-3 \alpha\left\langle r^{2} e^{i 2 \phi}\right\rangle_{u}-\left(\left\langle r e^{i \phi}\right\rangle_{e}-\alpha\langle\alpha\rangle_{u}\right)\right. \\
\left.\cdot\left(3\left(\left\langle r^{2} e^{i 2 \phi}\right\rangle_{e}-2 \alpha\left\langle r e^{i \phi}\right\rangle_{u}\right)-2\left(\left\langle r e^{i \phi}\right\rangle_{e}-\langle\alpha\rangle_{u}\right)^{2}\right)\right]
\end{aligned}
$$

\section{B.2 Including stress tensor}

Now, the generating function able to include effects of stress tensor only is

$$
\rho(\vec{x})=T^{\tau \tau}(\vec{x})-\beta \partial_{i} \partial_{j} T^{i j}(\vec{x}),
$$

and taking the Fourier transform, we have

$$
\rho(\vec{k})=\frac{1}{2 \pi} \int d^{2} x[T^{\tau \tau}-\beta \underbrace{\partial_{i} \partial_{j} T^{i j}}_{\text {II }}] e^{i \vec{k} \cdot \vec{x}},
$$

Its possible to solve II by parts as

$$
\begin{aligned}
& \mathrm{II}=\int \overbrace{d^{2} x \partial_{i} \partial_{j} T^{i j}}^{d v} \overbrace{e^{i \vec{k} \cdot \vec{x}}}^{u} \\
& =u v-\int v d u \\
& =\partial_{i}^{\left.T^{i j} e^{i \vec{k} \cdot \vec{x}}\right|_{-\infty} ^{+\infty} 0}-\int d^{2} x i k_{i} \partial_{j} T^{i j} e^{i \vec{k} \cdot \vec{x}} \\
& =-\underbrace{\int d^{2} x i k_{i} \partial_{j} T^{i j} e^{i \vec{k} \cdot \vec{x}}}_{\text {III }} .
\end{aligned}
$$

And III we solved by parts too as

$$
\begin{aligned}
& \mathrm{III}=\int \overbrace{d^{2} x \partial_{j} T^{i j}}^{d w} \overbrace{e^{i \vec{k} \cdot \vec{x}}}^{v} \\
& =v w-\int v d w \\
& =\left.T^{i j} e^{i \vec{k} \cdot \vec{x}}\right|_{-\infty} ^{+\infty}+\int d^{2} x i^{2} k_{i} k_{j} T^{i j} e^{i \vec{k} \cdot \vec{x}},
\end{aligned}
$$


and II is equal to

$$
\mathrm{II}=-\int d^{2} x k_{i} k_{j} T^{i j} e^{i \vec{k} \cdot \vec{x}} .
$$

Now, let us go back to Eq. (B.22), and later we will develop and simplify the contraction $k_{i} k_{j} T^{i j}$.

$$
\begin{aligned}
\rho(\vec{k}) & =\frac{1}{2 \pi} \int d^{2} x[T^{\tau \tau}-\beta \overbrace{k_{i} k_{j} T^{i j}}^{\mathrm{IV}}] e^{i \vec{k} \cdot \vec{x}} \\
& =\rho_{0}+\rho_{1}+\beta \rho_{2} .
\end{aligned}
$$

The contraction IV is well understand as

$$
\mathrm{IV}=k_{i} T^{i j} k_{j}=\left(\begin{array}{ll}
k_{x} & k_{y}
\end{array}\right)_{1 \times 2}\left(\begin{array}{cc}
T^{x x} & T^{x y} \\
T^{y x} & T^{y y}
\end{array}\right)_{2 \times 2}\left(\begin{array}{c}
k_{x} \\
k_{y}
\end{array}\right)_{2 \times 1},
$$

making the second contraction, we have

$$
I V=\left(\begin{array}{ll}
k_{x} & k_{y}
\end{array}\right)_{1 \times 2}\left(\begin{array}{c}
k_{x} T^{x x}+k_{y} T^{x y} \\
k_{x} T^{y x}+k_{y} T^{y y}
\end{array}\right)_{2 \times 1} .
$$

Then, IV simplified is

$$
\begin{gathered}
k_{i} k_{j} T^{i j}=K_{x}^{2} T^{x x}+k_{x} k_{y} T^{x y}+k_{x} k_{y} T^{y x}+k_{y}^{2} T^{y y} \\
\mathrm{IV}=K_{x}^{2} T^{x x}+2 k_{x} k_{y} T^{x y}+k_{y}^{2} T^{y y}, \\
\mathrm{IV}=k^{2} T^{x x} \cos ^{2} \phi_{k}+2 k^{2} T^{x y} \cos \phi_{k} \sin \phi_{k}+k^{2} T^{y y} \sin ^{2} \phi_{k} \\
=k^{2} T^{x x} \cos ^{2} \phi_{k}+k^{2} T^{x y} \sin 2 \phi_{k}+k^{2} T^{y y} \sin ^{2} \phi_{k}, \\
\mathrm{IV}=k^{2} T^{x x}\left(\frac{e^{i 2 \phi_{k}}+e^{-i 2 \phi_{k}}+1}{4}\right)+k^{2} T^{x y}\left(\frac{e^{i 2 \phi_{k}}-e^{-i 2 \phi_{k}}}{2 i}\right) \\
-k^{2} T^{y y}\left(\frac{e^{i 2 \phi_{k}}+e^{-i 2 \phi_{k}}-1}{4}\right)
\end{gathered}
$$

which is the same that

$$
\begin{aligned}
\mathrm{IV}= & \frac{1}{2} k^{2} e^{i 2 \phi_{k}} \underbrace{\left[\left(\frac{T^{x x}-T^{y y}}{2}\right)-i T^{x y}\right]}_{c^{*}}+\frac{1}{2} k^{2} e^{-i 2 \phi_{k}} \underbrace{\left[\left(\frac{T^{x x}-T^{y y}}{2}\right)+i T^{x y}\right]}_{c} \\
& +k^{2} \underbrace{\left(\frac{T^{x x}+T^{y y}}{2}\right)}_{\frac{T}{2}} .
\end{aligned}
$$


The form more simplified of $k_{i} k_{j} T^{i j}$ is

$$
k_{i} k_{j} T^{i j}=k^{2}\left(\frac{1}{2} e^{i 2 \phi_{k}} C^{*}(\vec{x})+\frac{1}{2} e^{-i 2 \phi_{k}} C(\vec{x})+\frac{T(\vec{x})}{2}\right) .
$$

Expanding in Maclaurin and Fourier series, we have

$$
\rho(\vec{k})=\sum_{m=0}^{\infty} \sum_{|n| \leq m}^{m} \rho_{n, m}(\beta) k^{m} e^{-i \phi_{k}} .
$$

We starting by expanding the Fourier transform in powers of $k$.

$$
\begin{aligned}
\rho(\vec{k}) & =\frac{1}{2 \pi} \int d^{2} x \sum_{m=0}^{\infty} \frac{1}{m !}(i \vec{k} \cdot \vec{x})^{m}\left[T^{\tau \tau}+\beta k_{i} k_{j} T^{i j}\right] \\
& =\frac{1}{2 \pi} \int d^{2} x \sum_{m=0}^{\infty} \frac{1}{m !}(i \vec{k} \cdot \vec{x})^{m}\left[T^{\tau \tau}+\beta k^{2}\left(\frac{C}{2} e^{-i 2 \phi_{k}}+\frac{C^{*}}{2} e^{i 2 \phi_{k}}+\frac{T}{2}\right)\right] \\
& =\sum_{m=0}^{\infty} k^{m} \frac{1}{2 \pi} \frac{i^{m}}{m !} \int d^{2} x r^{m} \cos ^{m}\left(\phi_{k}-\phi\right)\left[T^{\tau \tau}-\beta k^{2}\left(\frac{C}{2} e^{-i 2 \phi_{k}}+\frac{C^{*}}{2} e^{i 2 \phi_{k}}+\frac{T}{2}\right)\right] .
\end{aligned}
$$

The $k^{0}$ term is identical to the $\beta \rightarrow 0$ case.

$$
\begin{aligned}
\rho(\vec{k}) & =\rho_{0}+\rho_{1}+\sum_{m=2}^{\infty} k^{m}\left[\frac{i^{m}}{m !} \int d^{2} x r^{m} \cos ^{m}\left(\phi_{k}-\phi\right) T^{\tau \tau}\right. \\
& \left.+\beta \frac{i^{m-2}}{(m-2) !} \int d^{2} x r^{m-2} \cos ^{m-2}\left(\phi_{k}-\phi\right) k^{2}\left(\frac{C}{2} e^{-i 2 \phi_{k}}+\frac{C^{*}}{2} e^{i 2 \phi_{k}}+\frac{T}{2}\right)\right] .
\end{aligned}
$$

Matching the appropriate power of $k$ gives

$$
\begin{aligned}
\rho_{m}\left(\phi_{k}\right) & =\frac{i^{m}}{m !} \int d^{2} x r^{m} T^{\tau \tau} \cos ^{m}\left(\phi_{k}-\phi\right) \\
& -\beta \frac{i^{m}}{(m-2) !} \int d^{2} x r^{m-2} \cos ^{m-2}\left(\phi_{k}-\phi\right)\left(\frac{C}{2} e^{-i 2 \phi_{k}}+\frac{C^{*}}{2} e^{i 2 \phi_{k}}+\frac{T}{2}\right) .
\end{aligned}
$$

Projecting out the nth Fourier transform hasmonic gives

$$
\begin{gathered}
\rho_{n, m}=\int d \phi_{k} \rho_{m}\left(\rho_{k}\right) e^{i n \phi} \\
=\rho_{n, m}^{e}+\rho_{n, m}^{c}, \\
\rho_{n, m}^{\epsilon}=\frac{i^{m}}{2^{m}\left(\frac{m+n}{2}\right) !\left(\frac{m-n}{2}\right) !} \int d^{2} x r^{m} e^{i n \phi} T^{\tau \tau},
\end{gathered}
$$




$$
\begin{aligned}
& \rho_{n, m}^{c}=\frac{i^{m}}{2 \pi(m-2) !} \int d \phi_{k} d^{2} x r^{m-2} \cos ^{m-2}\left(\phi_{k}-\phi\right)\left(\frac{C}{2} e^{-i 2 \phi_{k}}+\frac{C^{*}}{2} e^{i 2 \phi_{k}}+\frac{T}{2}\right) e^{i n \phi_{k}} \\
& =\frac{i^{m}}{2 \pi(m-2) !} \int d \phi_{k} d^{2} x r^{m-2} \cos ^{m-2} \phi_{k}\left(\frac{C}{2} e^{-i 2\left(\phi_{k}+\phi\right)}+\frac{C^{*}}{2} e^{i 2\left(\phi_{k}+\phi\right)}+\frac{T}{2}\right) e^{i n\left(\phi_{k}+\phi\right)}
\end{aligned}
$$

$$
\begin{aligned}
& \rho_{n, m}^{c}=\frac{i^{m}}{2 \pi(m-2) !} \int d^{2} x r^{m-2} {\left[\frac{2 \pi}{2^{m-2}}\left(\begin{array}{c}
m-2 \\
\frac{m+n-4}{2}
\end{array}\right) \frac{C}{2} e^{i(n-2) \phi}\right.} \\
&+\frac{2 \pi}{2^{m-2}}\left(\begin{array}{c}
m-2 \\
\frac{m+n}{2}
\end{array}\right) \frac{C^{*}}{2} e^{i(n-2) \phi} \\
&\left.+\frac{2 \pi}{2^{m-2}}\left(\begin{array}{c}
m-2 \\
\frac{m+n-2}{2}
\end{array}\right) \frac{T}{2}\right], \\
& \rho_{n, m}^{c}=\frac{i^{m}}{(m-2) !} \int d^{2} x r^{m-2}\left[\frac{(m-2) !}{2^{m-2}\left(\frac{m+n-4}{2}\right) !\left(\frac{m-n}{2}\right) !} \frac{C}{2} e^{i(n-2) \phi}\right. \\
&+\frac{(m-2) !}{2^{m-2}\left(\frac{m+n}{2}\right) !\left(\frac{m-n-4}{2}\right) !} \frac{C^{*}}{2} e^{i(n-2) \phi} \\
&\left.+\frac{(m-2) !}{2^{m-2}\left(\frac{m+n-2}{2}\right) !\left(\frac{m-n-2}{2}\right) !} \frac{T}{2}\right]
\end{aligned}
$$

and finally

$$
\begin{aligned}
\rho_{n, m}^{c}=\frac{i^{m}}{2^{m}\left(\frac{m+n}{2}\right) !\left(\frac{m-n}{2}\right) !} \int d^{2} x r^{m-2} & {\left[(m+n)\left(\frac{m+n}{2}-1\right) \frac{C}{2} e^{i(n-2) \phi}\right.} \\
& +(m-n)\left(\frac{m-n}{2}-1\right) \frac{C^{*}}{2} e^{i(n+2) \phi} \\
& \left.+(m+n)(m-n) \frac{T}{2}\right]
\end{aligned}
$$


And now we have a general form for its moments can written as

$$
\begin{gathered}
\rho_{n, m}(\beta)=\frac{i^{m}}{2^{m}\left(\frac{m+n}{2}\right) !\left(\frac{m-n}{2}\right) !}\left[\int d^{2} x r^{m} e^{i n \phi} T^{\tau \tau}\right. \\
-\beta(m+n)\left(\frac{m+n}{2}-1\right) \int d^{2} x r^{m-2} e^{i(n-2) \phi} C \\
-\beta(m-n)\left(\frac{m-n}{2}-1\right) \int d^{2} x r^{m-2} e^{i(n+2) \phi} C^{*} \\
\left.-\beta(m+n)(m-n) \int d^{2} x r^{m-2} e^{i n \phi} \frac{T}{2}\right] .
\end{gathered}
$$

As the same way as made before, the cumulants can written as

$$
\begin{gathered}
\rho_{0,2}(\beta)=\frac{1}{2} \frac{i^{2}}{2 !}\left[\left\langle r^{2}\right\rangle_{e}-4\langle\beta\rangle_{t}-\left|\left\langle r e^{i \phi}\right\rangle_{e}\right|^{2}\right], \\
\rho_{2,2}(\beta)=\frac{1}{4} \frac{i^{2}}{2 !}\left[\left\langle r^{2} e^{i 2 \phi}\right\rangle_{e}-4\langle\beta\rangle_{c}-\left\langle r e^{i \phi}\right\rangle_{e}\right], \\
W_{1,3}(\beta)=\frac{1}{8} \frac{i^{3}}{2 !}\left[\left\langle r^{3} e^{i \phi}\right\rangle_{e}-4 \beta\left\langle r e^{-i \phi}\right\rangle_{c}-8 \beta\left\langle r e^{i \phi}\right\rangle_{t}-\left\langle r^{2} e^{i 2 \phi}\right\rangle_{e}\left\langle r e^{-i \phi}\right\rangle_{e}\right. \\
\left.-2\left\langle r^{2}\right\rangle_{e}\left\langle r e^{i \phi}\right\rangle_{e}+2\left\langle r e^{i \phi}\right\rangle_{e}^{2}\left\langle r e^{-i \phi}\right\rangle_{e}\right], \\
W_{3,3}(\beta)=\frac{1}{8} \frac{i^{3}}{3 !}\left[\left\langle r^{3} e^{i 3 \phi}\right\rangle_{e}-12 \beta\left\langle r e^{i \phi}\right\rangle_{c}\right. \\
\left.-\left\langle r e^{i \phi}\right\rangle_{e}\left(3\left\langle r^{2} e^{i 2 \phi}\right\rangle_{e}-2\left\langle r e^{i \phi}\right\rangle_{e}^{2}\right)\right],
\end{gathered}
$$

And the inclusion of both contributions together is trivially made with a sum of the two results. 


\section{Bibliography}

[1] Wojciech Florkowski. Phenomenology of ultra-relativistic heavy-ion collisions. World Scientific Publishing Company, 2010.

[2] Matthew D Schwartz. Quantum field theory and the standard model. Cambridge University Press, 2014.

[3] David J Gross and Frank Wilczek. Ultraviolet behavior of non-abelian gauge theories. Physical Review Letters, 30(26):1343, 1973.

[4] Dirk H Rischke. The quark-gluon plasma in equilibrium. Progress in Particle and Nuclear Physics, 52(1):197-296, 2004.

[5] Lokesh Kumar, STAR Collaboration, et al. Star results from the rhic beam energy scan-i. Nuclear Physics A, 904:256c-263c, 2013.

[6] Mark G Alford, Andreas Schmitt, Krishna Rajagopal, and Thomas Schäfer. Color superconductivity in dense quark matter. Reviews of Modern Physics, 80(4):1455, 2008.

[7] Szabolcs Borsányi, Zoltan Fodor, Christian Hoelbling, Sandor D Katz, Stefan Krieg, and Kalman K Szabo. Full result for the qcd equation of state with 2+ 1 flavors. Physics Letters B, 730:99-104, 2014.

[8] Jean Letessier and Johann Rafelski. Hadrons and quark-gluon plasma, volume 18. Cambridge University Press, 2002.

[9] R Derradi De Souza, Tomoi Koide, and Takeshi Kodama. Hydrodynamic approaches in relativistic heavy ion reactions. Progress in Particle and Nuclear Physics, 86:35-85, 2016.

[10] Edward Shuryak. Heavy ion collisions: achievements and challenges. arXiv preprint arXiv:1412.8393, 2014.

[11] Ulrich Heinz and Raimond Snellings. Collective flow and viscosity in relativistic heavy-ion collisions. Annual Review of Nuclear and Particle Science, 63:123-151, 2013.

[12] Thomas Schäfer and Derek Teaney. Nearly perfect fluidity: from cold atomic gases to hot quark gluon plasmas. Reports on Progress in Physics, 72(12):126001, 2009.

[13] Thomas Schaefer. Fluid dynamics and viscosity in strongly correlated fluids. Annual Review of Nuclear and Particle Science, 64:125-148, 2014. 
[14] Alex Kovner, Larry McLerran, and Heribert Weigert. Gluon production at high transverse momentum in the mclerran-venugopalan model of nuclear structure functions. Physical Review D, 52(7):3809, 1995.

[15] Alex Krasnitz and Raju Venugopalan. Initial energy density of gluons produced in very-high-energy nuclear collisions. Physical Review Letters, 84(19):4309, 2000.

[16] K Adcox, SS Adler, S Afanasiev, C Aidala, NN Ajitanand, Y Akiba, A Al-Jamel, J Alexander, R Amirikas, K Aoki, et al. Formation of dense partonic matter in relativistic nucleus-nucleus collisions at rhic: experimental evaluation by the phenix collaboration. Nuclear Physics A, 757(1-2):184-283, 2005.

[17] Kevin Dusling, Wei Li, and Björn Schenke. Novel collective phenomena in high-energy proton-proton and proton-nucleus collisions. International Journal of Modern Physics E, 25(01):1630002, 2016.

[18] John Adams, MM Aggarwal, Z Ahammed, J Amonett, BD Anderson, D Arkhipkin, GS Averichev, SK Badyal, Y Bai, J Balewski, et al. Experimental and theoretical challenges in the search for the quark-gluon plasma: The star collaboration's critical assessment of the evidence from rhic collisions. Nuclear Physics A, 757(1-2):102-183, 2005.

[19] Renato Critelli. Strongly coupled non-abelian plasmas in a magnetic field. arXiv preprint arXiv:1609.03104, 2016.

[20] Larry McLerran. The cgc and the glasma: Two lectures at the yukawa institute. Progress of Theoretical Physics Supplement, 187:17-30, 2011.

[21] Francois Gelis, Edmond Iancu, Jamal Jalilian-Marian, and Raju Venugopalan. The color glass condensate. Annual Review of Nuclear and Particle Science, 60:463-489, 2010.

[22] Brahms Collaboration et al. Quark gluon plasma an color glass condensate at rhic? the perspective from the brahms experiment. arXiv preprint nucl-ex/0410020, 2004.

[23] BB Back, MD Baker, M Ballintijn, DS Barton, B Becker, RR Betts, AA Bickley, $\mathrm{R}$ Bindel, A Budzanowski, W Busza, et al. The phobos perspective on discoveries at rhic. Nuclear Physics A, 757(1-2):28-101, 2005.

[24] R Hagedorn. How we got to qcd matter from the hadron side by trial and error. In Quark Matter'84, pages 53-76. Springer, 1985.

[25] A Majumder and M Van Leeuwen. The theory and phenomenology of perturbative qcd based jet quenching. Progress in Particle and Nuclear Physics, 66(1):41-92, 2011.

[26] Francesco D’Eramo, Hong Liu, and Krishna Rajagopal. Transverse momentum broadening and the jet quenching parameter, redux. Physical Review D, 84(6):065015, 2011. 
[27] Marco Panero, Kari Rummukainen, and Andreas Schäfer. Lattice study of the jet quenching parameter. Physical review letters, 112(16):162001, 2014.

[28] Peter F Kolb and Ulrich Heinz. Hydrodynamic description of ultrarelativistic heavy-ion collisions. In Quark-Gluon Plasma 3, pages 634-714. World Scientific, 2004.

[29] R. A. Lacey. talk at winter workshop on nuclear dynamics. Squaw Valley, CA, 2013.

[30] Charles Gale, Sangyong Jeon, Björn Schenke, Prithwish Tribedy, and Raju Venugopalan. Event-by-event anisotropic flow in heavy-ion collisions from combined yangmills and viscous fluid dynamics. Physical review letters, 110(1):012302, 2013.

[31] Stefan Floerchinger and Urs Achim Wiedemann. Mode-by-mode fluid dynamics for relativistic heavy ion collisions. Physics Letters B, 728:407-411, 2014.

[32] Derek Teaney and Li Yan. Triangularity and dipole asymmetry in relativistic heavy ion collisions. Physical Review C, 83(6):064904, 2011.

[33] B Alver, BB Back, MD Baker, M Ballintijn, DS Barton, RR Betts, AA Bickley, R Bindel, W Busza, A Carroll, et al. System size, energy, pseudorapidity, and centrality dependence of elliptic flow. Physical review letters, 98(24):242302, 2007.

[34] B Alver and G Roland. Collision-geometry fluctuations and triangular flow in heavyion collisions. Physical Review C, 81(5):054905, 2010.

[35] Fernando G Gardim, Frederique Grassi, Matthew Luzum, and Jean-Yves Ollitrault. Mapping the hydrodynamic response to the initial geometry in heavy-ion collisions. Physical Review C, 85(2):024908, 2012.

[36] Fernando G Gardim, Jacquelyn Noronha-Hostler, Matthew Luzum, and Frédérique Grassi. Viscous effects on the mapping of the initial to final state in heavy ion collisions. arXiv preprint arXiv:1411.2574, 2014.

[37] Mateusz Ploskon. Heavy-ion collisions-hot qcd in a lab. arXiv preprint arXiv:1808.01411, 2018.

[38] Wojciech Florkowski. Basic phenomenology for relativistic heavy-ion collisions. arXiv preprint arXiv:1410.7904, 2014.

[39] Jean-Yves Ollitrault. Relativistic hydrodynamics for heavy-ion collisions. European Journal of Physics, 29(2):275, 2008.

[40] Tetsufumi Hirano, Ulrich Heinz, Dmitri Kharzeev, Roy Lacey, and Yasushi Nara. Hadronic dissipative effects on elliptic flow in ultrarelativistic heavy-ion collisions. Physics Letters B, 636(6):299-304, 2006.

[41] Tetsufumi Hirano, Ulrich Heinz, Dmitri Kharzeev, Roy Lacey, and Yasushi Nara. Mass ordering of differential elliptic flow and its violation for $\phi$ mesons. Physical Review $C$, 77(4):044909, 2008. 
[42] D Teaney, J Lauret, and EV Shuryak. A hydrodynamic description of heavy ion collisions at the sps and rhic. arXiv preprint nucl-th/0110037, 2001.

[43] J Steinheimer, M Bleicher, H Petersen, G Burau, and H Stoecker. Strangeness production and local thermalization in an integrated boltzmann+ hydrodynamics approach. Acta Phys. Polon., 40:999-1004, 2009.

[44] Hannah Petersen and Marcus Bleicher. Ideal hydrodynamics and elliptic flow at cern super proton synchrotron (sps) energies: Importance of the initial conditions. Physical Review C, 79(5):054904, 2009.

[45] Klaus Werner, T Hirano, Iu Karpenko, T Pierog, S Porteboeuf, M Bleicher, and S Haussler. On the role of initial conditions and final state interactions in ultrarelativistic heavy ion collisions. Journal of Physics G: Nuclear and Particle Physics, 36(6):064030, 2009.

[46] Fred Cooper and Graham Frye. Single-particle distribution in the hydrodynamic and statistical thermodynamic models of multiparticle production. Physical Review D, 10(1):186, 1974.

[47] Jean-Fracois Paquet. Simulating heavy ion collisions with music documentation. webhome.phy.duke.edu/, 2018.

[48] Bjoern Schenke, Sangyong Jeon, and Charles Gale. (3+ 1) d hydrodynamic simulation of relativistic heavy-ion collisions. Physical Review C, 82(1):014903, 2010.

[49] Björn Schenke, Sangyong Jeon, and Charles Gale. Elliptic and triangular flow in eventby-event $\mathrm{d}=3+1$ viscous hydrodynamics. Physical review letters, 106(4):042301, 2011.

[50] Rodrigo Franco and Matthew Luzum. Rapidity-dependent eccentricity scaling in relativistic heavy-ion collisions. arXiv preprint arXiv:1910.14598, 2019.

[51] Hui Li and Li Yan. Pseudo-rapidity dependent hydrodynamic response in heavy-ion collisions. arXiv preprint arXiv:1907.10854, 2019.

[52] Pasi Huovinen and Péter Petreczky. Qcd equation of state and hadron resonance gas. Nuclear Physics A, 837(1-2):26-53, 2010.

[53] Rudolf Baier, Paul Romatschke, Dam Thanh Son, Andrei O Starinets, and Mikhail A Stephanov. Relativistic viscous hydrodynamics, conformal invariance, and holography. Journal of High Energy Physics, 2008(04):100, 2008.

[54] Paul Romatschke. New developments in relativistic viscous hydrodynamics. International Journal of Modern Physics E, 19(01):1-53, 2010.

[55] Werner Israel and JM Stewart. Transient relativistic thermodynamics and kinetic theory. Annals of Physics, 118(2):341-372, 1979. 
[56] Jacquelyn Noronha-Hostler, Gabriel S Denicol, Jorge Noronha, Rone PG Andrade, and Frederique Grassi. Bulk viscosity effects in event-by-event relativistic hydrodynamics. Physical Review C, 88(4):044916, 2013.

[57] Michael Strickland. Anisotropic hydrodynamics: three lectures. arXiv preprint arXiv:1410.5786, 2014.

[58] R mi Hakim. Introduction to relativistic statistical mechanics: classical and quantum. World scientific, 2011.

[59] Paul Romatschke and Ulrike Romatschke. Relativistic fluid dynamics in and out of equilibrium: and applications to relativistic nuclear collisions. Cambridge University Press, 2019.

[60] Yu M Sinyukov, SV Akkelin, Iu A Karpenko, and Y Hama. Kinetics vs hydrodynamics: generalization of landau/cooper-frye prescription for freeze-out. arXiv preprint arXiv:0901.1576, 2009.

[61] Sangwook Ryu, Sangyong Jeon, Charles Gale, Bjoern Schenke, and Clint Young. Music with the urqmd afterburner. Nuclear Physics A, 904:389c-392c, 2013.

[62] Bjoern Schenke, Prithwish Tribedy, and Raju Venugopalan. Fluctuating glasma initial conditions and flow in heavy ion collisions. Physical review letters, 108(25):252301, 2012.

[63] Bjoern Schenke, Prithwish Tribedy, and Raju Venugopalan. Event-by-event gluon multiplicity, energy density, and eccentricities in ultrarelativistic heavy-ion collisions. Physical Review C, 86(3):034908, 2012.

[64] Sergei A Voloshin, Arthur M Poskanzer, and Raimond Snellings. Collective phenomena in non-central nuclear collisions. In Relativistic Heavy Ion Physics, pages 293-333. Springer, 2010.

[65] Fernando G Gardim, Frédérique Grassi, Pedro Ishida, Matthew Luzum, Pablo S Magalhães, and Jacquelyn Noronha-Hostler. Probing the transverse size of initial inhomogeneities with flow observables. Nuclear Physics A, 982:419-422, 2019.

[66] Jean-Yves Ollitrault. Anisotropy as a signature of transverse collective flow. Physical Review D, 46(1):229, 1992.

[67] Jacquelyn Noronha-Hostler, Li Yan, Fernando G Gardim, and Jean-Yves Ollitrault. Linear and cubic response to the initial eccentricity in heavy-ion collisions. Physical Review C, 93(1):014909, 2016.

[68] Burak Han Alver, Clement Gombeaud, Matthew Luzum, and Jean-Yves Ollitrault. Triangular flow in hydrodynamics and transport theory. Physical Review C, 82(3):034913, 2010 . 
[69] S Manly, Phobos Collaboration, et al. System size, energy and pseudorapidity dependence of directed and elliptic flow at rhic. Nuclear Physics A, 774:523-526, 2006.

[70] Matthew Luzum and Paul Romatschke. Erratum: Conformal relativistic viscous hydrodynamics: Applications to rhic results at s nn= 200 gev [phys. rev. c 78, 034915 (2008)]. Physical Review C, 79(3):039903, 2009.

[71] Jonah E Bernhard. Bayesian parameter estimation for relativistic heavy-ion collisions. arXiv preprint arXiv:1804.06469, 2018.

[72] Mike Miller and Raimond Snellings. Eccentricity fluctuations and its possible effect on elliptic flow measurements. arXiv preprint nucl-ex/0312008, 2003. 